Fall 2011

\title{
Research and development studies for CMS at the Super LHC conditions and inclusive search for new physics at CMS with jets and missing momentum signature
}

Elif Asli Albayrak

University of Iowa

\section{Copyright 2011 ELIF ASLI ALBAYRAK}

This dissertation is available at Iowa Research Online: https://ir.uiowa.edu/etd/2666

\section{Recommended Citation}

Albayrak, Elif Asli. "Research and development studies for CMS at the Super LHC conditions and inclusive search for new physics at CMS with jets and missing momentum signature." $\mathrm{PhD}$ (Doctor of Philosophy) thesis, University of Iowa, 2011.

https://doi.org/10.17077/etd.d4s8nukk

Follow this and additional works at: https://ir.uiowa.edu/etd

Part of the Physics Commons 
RESEARCH AND DEVELOPMENT STUDIES FOR CMS AT THE SUPER LHC CONDITIONS

AND

INCLUSIVE SEARCH FOR NEW PHYSICS AT CMS WITH JETS AND

MISSING MOMENTUM SIGNATURE

by

Elif Asli Albayrak

\begin{abstract}
$\underline{\text { An Abstract }}$
Of a thesis submitted in partial fulfillment of the requirements for the Doctor of Philosophy

degree in Physics

in the Graduate College of

The University of Iowa
\end{abstract}

December 2011

Thesis Supervisor: Professor Yasar Onel 


\begin{abstract}
This thesis is composed of two independent parts. The first part is devoted to Hadronic Endcap Calorimeter R\&D studies for the Large Hadron Collider (LHC) higher luminosity conditions, whereas in the second part a search for new physics at the Compact Muon Solenoid (CMS) experiment is presented.

After a brief introduction to the LHC and the CMS experiment, in the first part R\&D studies on the active material of Hadronic Endcap (HE) Calorimeter for Compact Muon Solenoid Detector Upgrade are presented. We performed radiation damage and beam test studies on several wavelength shifter materials, and found that the p-terphenyl deposited quartz plates give improved light efficiency and they are also radiation hard. As a result we propose to substitute the current HE scintillator tiles with p-terphenyl deposited quartz plates for the higher luminosity era of the Large Hadron Collider.

In the second part, an inclusive search for new physics with the jets and missing momentum signature at the Large Hadron Collider is presented for $36 \mathrm{pb}^{-1}$ data collected by the Compact Muon Solenoid detector from March 2010 to November 2010. The emphasis was put on jet resolution measurements for the QCD background estimation with a photon-jet data sample. We did not observe an excess above the background, and thus derived upper limits in the context of R-parity conserving Constrained Minimal Supersymmetric Standard Model (CMSSM) and, more generally in the simplified model spectra. In CMSSM, depending on squark and gluino masses a 95\% confidence level (CL) upper limit for cross section of 2-3 pb is obtained, and gluino masses below $500 \mathrm{GeV}$ are excluded with 95\% CL for squarks with mass below $1 \mathrm{TeV}$. In simplified models, depending on the decay chain and mass of the new particles which are pair-produced and decay to either one or
\end{abstract}


two jets and a dark matter candidate, a 95\% CL upper limit on the production cross section in the $0.5-30 \mathrm{pb}$ range is obtained.

$\begin{aligned} & \text { Abstract Approved: } \\ & \\ & \text { Thesis Supervisor } \\ & \\ & \text { Date and Department }\end{aligned}$


RESEARCH AND DEVELOPMENT STUDIES FOR CMS AT THE SUPER LHC CONDITIONS

AND

INCLUSIVE SEARCH FOR NEW PHYSICS AT CMS WITH JETS AND

MISSING MOMENTUM SIGNATURE

by

Elif Asli Albayrak

A thesis submitted in partial fulfillment of the requirements for the Doctor of Philosophy

degree in Physics

in the Graduate College of

The University of Iowa

December 2011

Thesis Supervisor: Professor Yasar Onel 
Copyright by

ELIF ASLI ALBAYRAK

2011

All Rights Reserved 


\author{
Graduate College \\ The University of Iowa \\ Iowa City, Iowa
}

\title{
CERTIFICATE OF APPROVAL
}

PH.D. THESIS

This is to certify that the Ph.D. thesis of

\section{Elif Asli Albayrak}

has been approved by the Examining Committee for the thesis requirement for the Doctor of Philosophy degree in Physics at the December 2011 graduation.

Thesis Committee:

Yasar Onel, Thesis Supervisor

Jane M. Nachtman

Edwin Norbeck

Mary Hall Reno

Kenichi Hatakeyama 
To my family. 


\section{ACKNOWLEDGEMENTS}

First and foremost I want to thank my advisor Prof. Yasar Onel. I am deeply grateful for his support during my studies at University of Iowa and at CMS experiment.

I would like to also thank to the other members of my committee: Edwin Norbeck, Jane M. Nachtman, Mary Hall Reno, and Kenichi Hatakeyama. I very much appreciated their comments and suggestions.

I am indebted to my Fermilab LHC Physics Center colleagues Daniel Elvira, Marek Zielinski, and Seema Sharma for their guidance and support during the last year of my research at CMS experiment. I am especially grateful to Seema Sharma for all the discussion and advice during my resolution measurement study.

I would like to also thank to my friends and colleagues Firdevs Duru and Ugur Akgun who made Iowa City a very special place for me. I am grateful for the time I spent with them.

This work would not have been possible without my family. Despite the geographical distance, I always felt their love and support.

Last but not least, I am grateful to my encouraging husband Taylan Yetkin

for his constant love and support. His patience during the final stage of this $\mathrm{PhD}$ is invaluable to me. 


\begin{abstract}
This thesis is composed of two independent parts. The first part is devoted to Hadronic Endcap Calorimeter R\&D studies for the Large Hadron Collider (LHC) higher luminosity conditions, whereas in the second part a search for new physics at the Compact Muon Solenoid (CMS) experiment is presented.

After a brief introduction to the LHC and the CMS experiment, in the first part R\&D studies on the active material of Hadronic Endcap (HE) Calorimeter for Compact Muon Solenoid Detector Upgrade are presented. We performed radiation damage and beam test studies on several wavelength shifter materials, and found that the p-terphenyl deposited quartz plates give improved light efficiency and they are also radiation hard. As a result we propose to substitute the current HE scintillator tiles with p-terphenyl deposited quartz plates for the higher luminosity era of the Large Hadron Collider.
\end{abstract}

In the second part, an inclusive search for new physics with the jets and missing momentum signature at the Large Hadron Collider is presented for $36 \mathrm{pb}^{-1}$ data collected by the Compact Muon Solenoid detector from March 2010 to November 2010. The emphasis was put on jet resolution measurements for the QCD background estimation with a photon-jet data sample. We did not observe an excess above the background, and thus derived upper limits in the context of R-parity conserving Constrained Minimal Supersymmetric Standard Model (CMSSM) and, more generally in the simplified model spectra. In CMSSM, depending on squark and gluino masses a 
$95 \%$ confidence level (CL) upper limit for cross section of $2-3 \mathrm{pb}$ is obtained, and gluino masses below $500 \mathrm{GeV}$ are excluded with $95 \%$ CL for squarks with mass below $1 \mathrm{TeV}$. In simplified models, depending on the decay chain and mass of the new particles which are pair-produced and decay to either one or two jets and a dark matter candidate, a 95\% CL upper limit on the production cross section in the $0.5-30 \mathrm{pb}$ range is obtained. 


\section{TABLE OF CONTENTS}

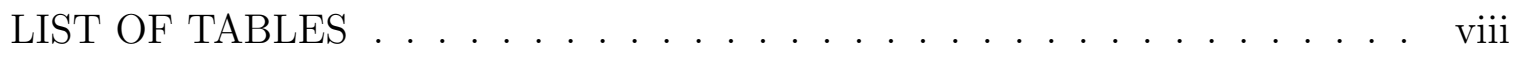

LIST OF FIGURES . . . . . . . . . . . . . . . . . CHAPTER

1 INTRODUCTION . . . . . . . . . . . . . . . . . 1

1.1 New Physics at Large Hadron Collider . . . . . . . . . . . . . . . 1

1.2 What Is Next After Discovery? . . . . . . . . . . . . . . 5

2 THE LARGE HADRON COLLIDER AND THE COMPACT MUON SOLENOID EXPERIMENT . . . . . . . . . . . . . 6

2.1 The Large Hadron Collider . . . . . . . . . . . . . . . . . . . . 6

2.2 The Compact Muon Solenoid Experiment . . . . . . . . . . 8

2.2.1 The Magnet . . . . . . . . . . . . . . . . . . . 10

2.2.2 The Muon System . . . . . . . . . . . . . . . 10

2.2.3 The Tracker . . . . . . . . . . . . . . . . . 12

2.2.4 The Calorimeters . . . . . . . . . . . . . . 15

3 R\&D STUDIES FOR HE UPGRADE AT CMS . . . . . . . . . . . . 18

3.1 Introduction . . . . . . . . . . . . . . . . . . 18

3.2 Hadronic Endcap Calorimeter and Radiation Damage . . . . . . 20

3.3 Selection of Wavelength Shifter . . . . . . . . . . . . . . . . . . . 22

3.4 Calorimetric Properties of Coated Quartz Plates . . . . . . . . . 26

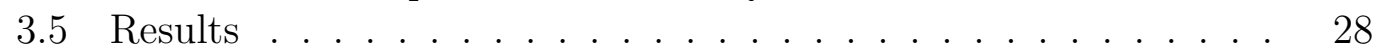

3.6 Conclusion and Future Plans . . . . . . . . . . . . . . . 33

4 INCLUSIVE SEARCH FOR NEW PHYSICS AT CMS WITH JETS AND MISSING MOMENTUM SIGNATURE . . . . . . . . . . . . . . 37

4.1 Introduction . . . . . . . . . . . . . . . . . . . 37

4.2 SUSY Search at the LHC with CMS . . . . . . . . . . . . . . . . . . . . 41

4.3 Event Reconstruction in CMS . . . . . . . . . . . . . . . . . 46

4.4 Sample Selection . . . . . . . . . . . . . . . . . . . . . . 46

4.5 Monte Carlo Production . . . . . . . . . . . . . . . . . . . . . . 49

4.6 The SM Backgrounds . . . . . . . . . . . . . . . 50

4.6.1 Z $(\nu \bar{\nu})+$ jets Background Estimation . . . . . . . . . 50 
4.6.2 $\mathrm{W}+$ jets and $t \bar{t}$ Background Estimation . . . . . . . 53

4.6.2.1 The $\mathrm{W} / t \bar{t} \rightarrow e, \mu+\mathrm{X}$ background estimation . . . 53

4.6.2.2 The $\mathrm{W} / t \bar{t} \rightarrow \tau_{h}+\mathrm{X}$ background estimation . . . 55

5 QCD BACKGROUND ESTIMATION . . . . . . . . . . 61

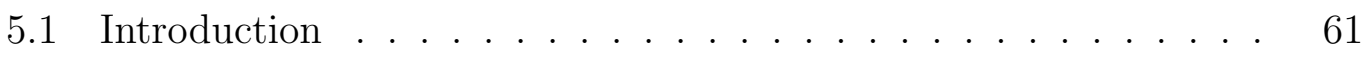

5.2 Jet Energy Resolution Measurements _. . . . . . . . . . . . 63

$5.2 .1 \gamma$ + Jet Measurements . . . . . . . . . . . . . 65

5.2.1.1 Dataset and Event Selection . . . . . . . . . 66

5.2.1.2 Measurement of Resolutions in Data and MC . . 68

5.2.1.3 Estimation of non-Gaussian Component . . . . . 77

5.2 .2 Dijet Measurements . . . . . . . . . . . . . . . 80

5.2.3 Systematic Uncertainty Measurements . . . . . . . . . . 83

5.2.3.1 Uncertainty Due to Extrapolation Fit Range . . 85

5.2.3.2 Uncertainty Due to $\Delta \phi(\gamma$, jet1) Requirement . . 86

5.2.3.3 Uncertainty Due to Jet Energy Corrections . . . 87

5.2.3.4 Uncertainty Due to Imbalance Component . . . 88

5.2.3.5 Uncertainty Due to Flavor Composition in QCD and $\gamma+$ jet Samples: . . . . . . . . . . . . 91

5.2.3.6 Uncertainty Due to Pileup . . . . . . . . . . . . 93

5.3 Results of the Rebalance and Smear Method . . . . . . . . 95

6 RESULTS OF INCLUSIVE SEARCH FOR NEW PHYSICS . . . . 106

6.1 Results and Limits . . . . . . . . . . . . . . . . . 106

6.2 Interpretation within the CMSSM . . . . . . . . . . 108

6.3 Interpretation with Simplified Models . . . . . . . . . . . . 109

6.4 Conclusion . . . . . . . . . . . . . . . . . . . 117

7 SUMMARY AND CONCLUSION . . . . . . . . . . . . . 119

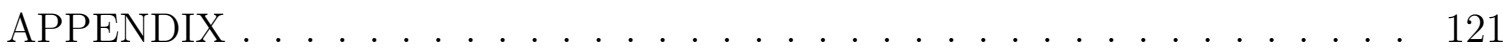

A EFFECT OF LOWER JET P $P_{T}$ LIMIT ON RESOLUTION MEASURE-

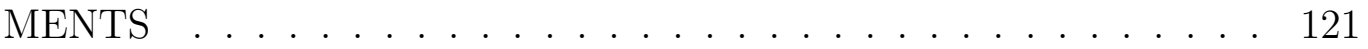

B THE EFFECT OF $\triangle$ R MATCHING ON INTRINSIC RESOLUTION 127

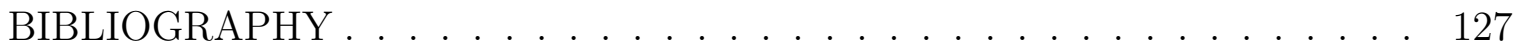




\section{LIST OF TABLES}

Table

4.1 The MSSM chiral and gauge supermultiplets. . . . . . . . . . . . . . 39

4.2 The CMSSM mass points at CMS. . . . . . . . . . . . . . . . 42

4.3 List of the MC datasets and corresponding cross sections. . . . . . . . . . 49

4.4 Event yields in data and MC samples for the largest SM backgrounds. . . 51

4.5 The number of $\gamma+$ jets events in data and estimated number of $\mathrm{Z}(\nu \bar{\nu})+$ jets background. . . . . . . . . . . . . . . . 52

4.6 The estimated number of events for the lost lepton background when the number is extracted from the data or MC. . . . . . . . . . . . . 55

4.7 Estimated number of events for the hadronic $\tau$ background from data and MC simulations with their statistical and systematic uncertainties. . . . .

5.1 Number of events predicted after search selection estimated from PYTHIA multijet sample. . . . . . . . . . . . . . . . . . .

5.2 Photon HLT paths and corresponding photon $\mathrm{p}_{\mathrm{T}}$ bins used in jet resolution measurements. . . . . . . . . . . . . . . .

5.3 Ratio of the resolutions measured in data and MC for different jet $\eta$ ranges using the unbinned likelihood fits. . . . . . . . . . . . . . . .

5.4 Relative changes in the measured Data/MC ratios due to mismeasurement of the MC imbalance component and the assigned systematic uncertainties. 92

5.5 Data/MC ratio of the measured intrinsic resolutions with (withPUcorr) and without pileup (noPUcorr) correction on data and assigned uncertainties for different jet $\eta$ ranges. . . . . . . . . . . . . . . . . . . .

5.6 Data/MC ratio of the measured intrinsic resolutions and assigned uncertainties for different jet $\eta$ ranges. . . . . . . . . . . . . . . . .

5.7 Number of QCD multijet events predicted before and after bias corrections. 96 
6.1 Predicted number of events from different background components. . . . 106 


\section{LIST OF FIGURES}

Figure

2.1 A schematic view of the CERN accelerator complex including the LHC, and LHC experiments. . . . . . . . . . . . . . . . . . . 7

2.2 Three dimensional view of CMS . . . . . . . . . . . . . . . . . 9

2.3 Coil of the superconducting magnet. . . . . . . . . . . . . . . . 11

2.4 The one quarter layout of the CMS muon system. . . . . . . . . . . . . 13

2.5 Transverse view of the Barrel Muon detector. . . . . . . . . . . . . . . . 14

2.6 The tracker layout $(1 / 4$ of the $\mathrm{z}$ view $) . \ldots \ldots$. . . . . . . . 14

2.7 Layout of pixel detectors in the CMS tracker. . . . . . . . . . . . 16

3.1 Total integrated luminosity recorded in CMS during 2010. . . . . . . . . 19

3.2 Hadron endcap calorimeter mounted on the endcap iron yoke. . . . . . . 21

3.3 Fermilab Thin Film Laboratory vacuum chamber (pTp evaporating system). 23

3.4 Fermilab Thin Film Labarotary ZnO:Ga sputtering system and guns. . . 24

3.5 The comparison of single photoelectron level signal distributions from $2 \mu \mathrm{m}$ thickness of pTp (red solid line), and $0.2 \mu \mathrm{m}$ of $\mathrm{ZnO}$ :Ga (black solid line) deposited plates to the plain quartz plate (dashed blue line). . . . . . . . 25

3.6 The WLS deposited quartz plate light output test setup. . . . . . . . . . 26

3.7 Light output from pTp sample after proton irradiation versus proton irradiation level with simple fitted line. . . . . . . . . . . . . . 27

3.8 The calorimeter prototype in the electromagnetic configuration. . . . . . 29

3.9 Longitudinal shower profile for different pion energies. . . . . . . . . . . . 30

$3.10300 \mathrm{GeV}$ pion response of pTp deposited quartz plate calorimeter. . . . . 31 
3.11 Hadronic detector linearity and energy resolution, data (red circle and solid line) and Geant4 simulations (blue square and solid line), for the calorimeter prototype.

3.12 Longitudinal shower profile for different electron energies. . . . . . . . . . 33

$3.13100 \mathrm{GeV}$ electron response of the calorimeter prototype. . . . . . . . . . 34

3.14 Electromagnetic detector linearity and energy resolution, data (red circle and solid line) and Geant4 simulations (blue square and dashed line), for the calorimeter prototype. . . . . . . . . . . . . . .

4.1 Feynman diagrams for $\tilde{q} \tilde{q}(\mathrm{a}), \tilde{q} \tilde{g}(\mathrm{~b})$, and $\tilde{g} \tilde{g}(\mathrm{c})$ productions. . . . . . . 58

4.2 A typical example for $\tilde{g} \rightarrow \tilde{q} q$ decay with multijets (quarks and taus) and large missing transverse energy $\left(\tilde{\chi}_{1}^{0}\right)$ final state for CMS LM1 benchmark point. . . . . . . . . . . . . . . . . .

4.3 $\Delta \phi$ distributions between the first three leading jets and the $H_{\mathrm{T}}$ for the QCD and LM1 samples. All histograms are normalized to unit area. . . .

4.4 $\mathrm{H}_{\mathrm{T}}$ (left) and $\mathrm{H}_{\mathrm{T}}$ (right) distributions for the data and $\mathrm{MC}$ samples after baseline selections except $H_{\mathrm{T}}>150 \mathrm{GeV}$ requirement. . . . . . . . .

4.5 $\mathrm{H}_{\mathrm{T}}$ (left) and $\mathrm{H}_{\mathrm{T}}$ (right) distributions for the data and $\mathrm{MC}$ samples after baseline selections including the $H_{\mathrm{T}}>150 \mathrm{GeV}$ requirement. . . . . . .

5.1 The $H_{\mathrm{T}}$ (left) and $\mathrm{H}_{\mathrm{T}}$ (right) distributions from the rebalance and smear method applied to simulation events and compared to actual MC (MC truth) distributions for events passing multijet, $\mathrm{H}_{\mathrm{T}} \geq 300 \mathrm{GeV}$, and $\Delta \phi\left(\right.$ jet $\left._{1-3}, H_{\mathrm{T}}\right)$

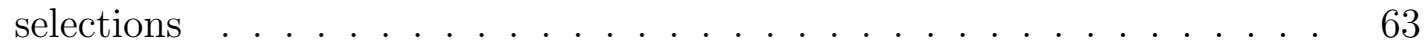

5.2 Photon $\mathrm{p}_{\mathrm{T}}$ distribution collected with different photon HLT paths. . . . . 67

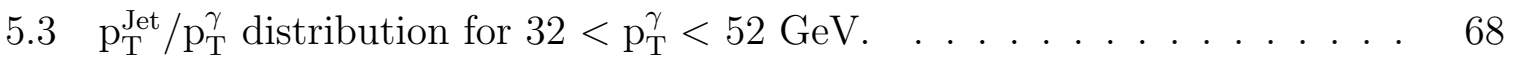

5.4 Imbalance distributions for $140-180 \mathrm{GeV}$ photon $\mathrm{p}_{\mathrm{T}}$ bin. . . . . . . . . . 69

5.5 Jet resolution functions for $140-180 \mathrm{GeV}$ photon $\mathrm{p}_{\mathrm{T}}$ bin. . . . . . . . . . 71

5.6 Measured (left) and intrinsic (right) resolutions in Data (red) and MC (blue) in the $90-110 \mathrm{GeV}$ photon $\mathrm{p}_{\mathrm{T}}$ bin for $0.0<|\eta|<1.1$ region. . . . 72 
5.7 Intrinsic resolutions measured in Data (red) and MC (blue) for the photon $\mathrm{p}_{\mathrm{T}}$ bins $(22-32),(32-52)$, and $(52-72) \mathrm{GeV}$ for the $|\eta|<1.1$ region. Distributions are shown for lower (left) and higher (right) extrapolation

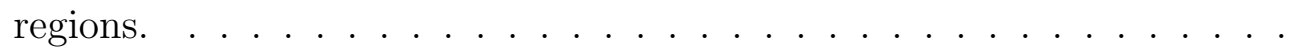

5.8 Intrinsic resolutions measured in Data (red) and MC (blue) for the photon $\mathrm{p}_{\mathrm{T}}$ bins $(72-90),(90-110)$, and $(110-140) \mathrm{GeV}$ for the $|\eta|<1.1$ region. Distributions are shown for lower (left) and higher (right) extrapolation regions. . . . . . . . . . . . . . . . . . . . . . . . .

5.9 Ratio of intrinsic resolutions measured in Data and $\mathrm{MC}$ for the photon $\mathrm{p}_{\mathrm{T}}$ bins $(22-32),(32-52)$, and $(52-72) \mathrm{GeV}$ for the $|\eta|<1.1$ region. Distributions are shown for lower (left) and higher (right) extrapolation

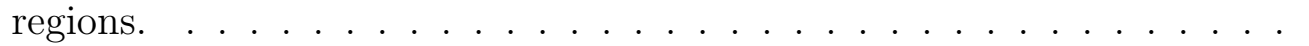

5.10 Ratio of intrinsic resolutions measured in Data and $\mathrm{MC}$ for the photon $\mathrm{p}_{\mathrm{T}}$ bins $(72-90),(90-110)$, and $(110-140) \mathrm{GeV}$ for the $|\eta|<1.1$ region. Distributions are shown for lower (left) and higher (right) extrapolation regions. . . . . . . . . . . . . . .

5.11 Extrapolated ratio of the intrinsic resolution measured in data and MC for the various bins of jet $\eta \ldots \ldots \ldots \ldots . \ldots \ldots$

5.12 Measured jet $\mathrm{p}_{\mathrm{T}}$ resolution functions in $\mathrm{MC}$ and data for different photon $\mathrm{p}_{\mathrm{T}}$ bins for $|\eta|<1.1 \ldots \ldots \ldots \ldots \ldots \ldots \ldots \ldots \ldots$

5.13 Ratio of the number of tail events in data and $\mathrm{MC}$ vs $\mathrm{p}_{\mathrm{T}}^{\gamma} \ldots \ldots \ldots . . .80$

5.14 The ratio of jet $\mathrm{p}_{\mathrm{T}}$ resolutions in data and $\mathrm{MC}$ samples versus jet $\mathrm{p}_{\mathrm{T}}$, in $|\eta|<1.1$, from dijet and $\gamma+$ jet samples. . . . . . . . . . 81

5.15 Results from the combination fits versus jet $\mathrm{p}_{\mathrm{T}}$ in various $\eta$ ranges. . . .

5.16 The Data/MC correction factor for the jet resolution tails observed in dijet samples. . . . . . . . . . . . . . . . . .

5.17 Data/MC ratio for nominal (black), lower extrapolation range (blue), and higher extrapolation range (red) measurements for the $0.0<|\eta|<1.1$ region. . . . . . . . . . . . . . . . .

5.18 Ratio of intrinsic resolutions measured in $\mathrm{MC}$ for two different $\Delta \phi$ requirements for $0.0<|\eta|<1.1$ region. . . . . . . . . . . . 
5.19 Data/MC ratio for nominal (black), JEC up (blue), and JEC down (red) measurements for $0.0<|\eta|<1.1$ region. . . . . . . . . . . .

5.20 Data/MC ratios for intrinsic resolution for different MC generators; PYTHIA (a), MADGRAPH (b), and HERWIG (c). The distributions are shown for pseudorapidity range $0.0<|\eta|<1.1 \ldots \ldots \ldots \ldots$. . . . . . 97

5.21 Measured imbalance resolutions for data and MC . . . . . . . . . . . 98

5.22 Data/MC ratio for intrinsic resolutions measured from PYTHIA with and without hadronization. . . . . . . . . . . . . . .

5.23 Measured imbalance resolutions in the fraction of $\mathrm{p}_{\mathrm{T}}^{\mathrm{Jet} 2} / \mathrm{p}_{\mathrm{T}}^{\gamma}$ for $22-32$, $52-72 \mathrm{GeV}$ photon $\mathrm{p}_{\mathrm{T}}$ bins. . . . . . . . . . . . . . . 100

5.24 Data/MC ratio for intrinsic resolutions measured by using PYTHIA with (right) and without (left) $k_{T}$ smearing. The distributions are shown for pseudorapidity range $0.0<|\eta|<1.1 \ldots \ldots \ldots$. . . . . . . . 101

5.25 Intrinsic resolutions (top) for $\mathrm{QCD}$ and $\gamma+$ jet samples with respect to particle level jet $\mathrm{p}_{\mathrm{T}}$ for the $0.0<|\eta|<1.1$ region and the ratio of $\sigma_{\text {photon }}$ $/ \sigma_{\mathrm{QCD}}$ (bottom). . . . . . . . . . . . . . . . 102

5.26 Measured resolutions in the fraction of $\mathrm{p}_{\mathrm{T}}^{\mathrm{Jet} 2} / \mathrm{p}_{\mathrm{T}}^{\gamma} \ldots \ldots \ldots \ldots \ldots$

5.27 Intrinsic resolutions in the fraction of $\mathrm{p}_{\mathrm{T}}^{\mathrm{Jet} 2} / \mathrm{p}_{\mathrm{T}}^{\gamma}$ for $22-32 \mathrm{GeV}$ (left), $52-72$ GeV (right) photon $\mathrm{p}_{\mathrm{T}}$ bins. . . . . . . . . . . . . . 103

5.28 Data/MC ratio for without (left) and with (right) pileup correction in data

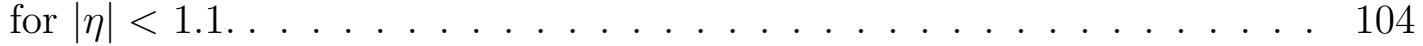

5.29 Extrapolated ratio of the intrinsic resolutions measured in data and Monte Carlo for various bins of jet $\eta$. . . . . . . . . . . . . . . . . . 105

6.1 Signal efficiency for High- $h_{\mathrm{T}}$ (left) and High- $\mathrm{H}_{\mathrm{T}}$ (right) selections $\ldots . .108$

6.2 Measured and expected 95\% CL exclusion contour at NLO in the CMSSM $m_{0}-m_{1 / 2}$ mass planes . . . . . . . . . . . . . 110

6.3 The 95\% CL upper limits in the CMSSM gluino-squark mass planes for LO and NLO cross sections. . . . . . . . . . . . . . . . . . . . . 111

6.4 Simplified model diagrams. Left: gluino pair production; right: squark pair production. . . . . . . . . . . . . . . . . . . . 112 
6.5 High- $\bigsqcup_{\mathrm{T}}$ (left) and High- $\mathrm{H}_{\mathrm{T}}$ (right) selection efficiencies for gluino production as a function of gluino and LSP mass. . . . . . . . . . . . . . . .

6.6 High- $h_{\mathrm{T}}$ (left) and High- $\mathrm{H}_{\mathrm{T}}$ (right) selection efficiencies for squark production as a function of squark and LSP mass. . . . . . . . . . . . . . . 114

6.7 Experimental uncertainty on the High- $h_{\mathrm{T}}$ (left) and High- $\mathrm{H}_{\mathrm{T}}$ (right) selection efficiency for gluino production as a function of the gluino and LSP

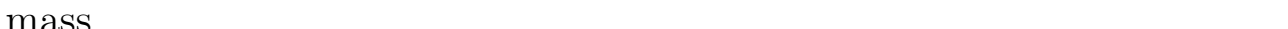

6.8 Experimental uncertainty on the High- $\boldsymbol{H}_{\mathrm{T}}$ (left) and High- $\mathrm{H}_{\mathrm{T}}$ (right) selection efficiency for squark production as a function of squark and LSP mass. . . . . . . . . . . . . . . . . . . .

6.9 Theoretical uncertainty on the High- $\boldsymbol{H}_{\mathrm{T}}$ (left) and High- $\mathrm{H}_{\mathrm{T}}$ (right) selection efficiency for gluino production as a function of the gluino and LSP mass. . . . . . . . . . . . . . . . . . . . 116

6.10 Theoretical uncertainty on the High- $\boldsymbol{H}_{\mathrm{T}}$ (left) and High- $\mathrm{H}_{\mathrm{T}}$ (right) selection efficiency for squark production as a function of squark and LSP mass.116

6.11 The 95\% CL upper limits for gluino pair production cross sections for High- $H_{\mathrm{T}}$ (left) and High- $\mathrm{H}_{\mathrm{T}}$ (right) selections as a function of the gluino and LSP mass. . . . . . . . . . . . . . . . . . . . . 117

6.12 The 95\% CL upper limits for squark pair production cross sections for High- $\breve{A}_{\mathrm{T}}$ (left) and High- $\mathrm{H}_{\mathrm{T}}$ (right) selections as function of squark and LSP mass. . . . . . . . . . . . . . . . . . . . . . . . . .

A.1 Comparison of nominal $H_{\mathrm{T}}$ (black) and estimated $\not_{\mathrm{T}}$ (blue) distributions. Particle jets with $p_{T}<20 \mathrm{GeV}$ are not smeared. Top plot shows distributions whereas the bottom plot shows their ratio. . . . . . . . . . . . . .

A.2 Comparison of nominal $h_{\mathrm{T}}$ (black) and estimated $\boldsymbol{H}_{\mathrm{T}}$ (red) distributions. Particle jets with $p_{T}<42 \mathrm{GeV}$ are not smeared. Top plot shows distributions whereas the bottom plot shows their ratio. . . . . . . . . . .

A.3 Comparison of nominal $h_{\mathrm{T}}$ (black) and estimated $h_{\mathrm{T}}$ (green) distributions. Particle jets with $p_{T}<62 \mathrm{GeV}$ are not smeared. Top plot shows distributions whereas the bottom plot shows their ratio. . . . . . . . . 125

A.4 Comparison of nominal $h_{\mathrm{T}}$ (black) and estimated $\not_{\mathrm{T}}$ (violet) distributions. Particle jets with $p_{T}<87 \mathrm{GeV}$ are not smeared. Top plot shows distributions whereas the bottom plot shows their ratio. 
B.1 Effect of varying the $\Delta \mathrm{R}$ matching between leading reconstructed jet and particle jet on intrinsic resolution as a function of particle jet $\mathrm{p}_{\mathrm{T}}$ for the four different eta bins (a) $0.0<|\eta|<1.1$, (b) $1.1<|\eta|<1.7$, (c) $1.7<$ $|\eta|<2.3$, (d) $2.3<|\eta|<5.0 \ldots \ldots \ldots \ldots \ldots \ldots$ 


\section{CHAPTER 1 INTRODUCTION}

This thesis has two independent parts. After a brief introduction to the LHC and the Compact Muon Solenoid (CMS) Experiment (Chapter 2), in the first part R\&D studies for CMS HE Calorimeters are presented (Chapter 3). In the second part, an inclusive search for new physics at CMS with jets and large missing momentum signature is presented (Chapter 4, 5, and 6).

\subsection{New Physics at Large Hadron Collider}

The Standard Model (SM) is a theory which developed in the early 1970s to describe the observed particles and the fundamental interactions between them. The SM predictions were tested by the previous experiments, and agreement with data is outstanding. The discovery of $\mathrm{W}$ and $\mathrm{Z}$ bosons, the observation of gluons and the precise measurements of SM predictions almost complete the understanding of nature based on the SM. As a result of highly accurate experimental observations, the SM became the most established and well tested physics theory.

Although the SM gives the best description of nature, it does not serve as a complete theory without introducing new physics models beyond it. The SM cannot incorporate gravity and cannot explain the source of dark matter in the universe. The most important missing part in the SM is the source of particle masses. The Higgs boson, via symmetry breaking, can explain the masses of the fundamental particles, but it has not been observed yet. Finding the Higgs boson is a big step, 
but this does not complete the picture. It is believed that the SM is a lower limit of a more complete model as it has shortcomings for describing all the experimental and cosmological data we have collected. With the new generation of collider experiments we are trying to observe signals of new physics and new particles. The Large Hadron Collider (LHC) [1] is built for this purpose, and it will explore the $\mathrm{TeV}$ range physics (physics beyond the SM), and experiments which use collision data to try to address the questions which cannot be answered by the SM.

With $14 \mathrm{TeV}$ center of mass energy collisions and high collision rates, the LHC will be able to discover the Higgs boson (if it exists), solve the mystery of dark matter in the universe and explore the physics beyond the SM. There are many beyond the SM theories, such as supersymmetry, extra dimensions, technicolor, which try to complete the missing pieces in our understanding of the universe. Supersymmetry (SUSY) is one of the many models which is the favorite of many theorists and experimentalists, as it also opens the door for unifying gravity with the other three forces.

The Minimally supersymmetric model (MSSM) is the supersymmetric extension of the SM with minimum particle content and the constrained MSSM (CMSSM) introduces new constraints and simplifies the unification of the forces at a higher mass scale. The LHC will be able to cover some part of this high energy region, and experiments will be able to discover some of the new particles if they have masses $\sim 1$ $\mathrm{TeV}$. The signature of the new physics, such as the case of R parity conserving SUSY, will manifest itself with multijets and a large missing energy final state. Therefore, 
to discover SUSY at the LHC it is important to perform an inclusive search based on a jets and large missing momentum signature. The analysis given in this thesis outlines this effort by using Compact Muon Solenoid (CMS) [2] data collected in 2010 collisions.

The LHC produces collisions for experiments in unprecedented energy (pp collisions at $\sqrt{\mathrm{s}}=7 \mathrm{TeV}$ ), rate, and high pileup (additional large number of soft pp collisions) conditions. Although many factors of the CMS detector are understood during commissioning with cosmic muon data, there are some which have to be studied with the collision data. In addition to that, new or improved analysis methods have to be developed for the new era. The discovery process requires a very good understanding of the detector and Standard Model backgrounds. The detector effects can result in mimicking the real signal, therefore it severely affects discovery potentials. Different procedures were developed to minimize, if not completely remove, these effects. Since most of the Monte Carlo (MC) generators we use to simulate collision events are tested and validated for the Fermilab Tevatron data ( $\mathrm{p} \overline{\mathrm{p}}$ collisions at $\sqrt{\mathrm{s}}=2 \mathrm{TeV}$ ) we need SM background estimations based on data. Many novel methods were developed for this purpose. All of these were achieved with the collaboration of many people, where each individual played a major role for understanding pieces that fit together to form the experimental results from the CMS detector.

The analysis summarized in chapters 4,5 , and 6 of this thesis is an outcome of group's work. The results are derived in a cut-based manner after combining efforts on detector noise cleanup and filtering algorithms, jet resolution measurements, data 
driven background estimations, uncertainty calculations, and finally interpretation of the results.

In this analysis, my contributions were in measuring the jet energy resolution with the $\gamma+$ jet $\mathrm{p}_{\mathrm{T}}$ balance technique and determining the experimental uncertainties in so-called simplified models. The results I produced from jet resolution measurements are combined with the dijet asymmetry method and used as a crucial input in the QCD background estimation method (Rebalance and Smear, R\&S). QCD is the biggest background in the analysis and the $\mathrm{R} \& \mathrm{~S}$ method is based on rebalancing multijet events by using jet resolution functions and then smearing those rebalanced events (seed sample) by using the full jet resolution functions. It is crucial to measure the jet resolution functions for the R\&S method to work. Apart from the background estimation method, the jet resolution measurements were also part of the CMS jet resolution measurement effort and the results have been submitted to JINST and will be published as "Determination of Jet Energy Calibration and Transverse Momentum Resolution in CMS." The combined jet resolutions are also used to estimate systematic uncertainties in the analysis and simplified model searches. These studies are given in detail in this thesis. For the completeness of the study the event selections, results from background estimations, and limit setting procedure are also summarized with the necessary references. 


\subsection{What Is Next After Discovery?}

If/when we discover the SUSY, the LHC will continue running and collecting more events to complete the SUSY particle mass spectrum and/or to study rare events such as the MSSM Higgs boson. With the luminosity conditions expected before a major upgrade it is not possible to collect enough data to observe rare events. The LHC has upgrade plans in two phases to increase luminosity, and during the second phase the detectors located at the LHC will undergo major upgrades due to high radiation damage caused by increased luminosity. The scintillator tiles used in the CMS Hadronic Endcap (HE) Detector will need to be replaced since with high radiation they will lose their light collection efficiency. As a solution to this problem we performed $R \& D$ studies on p-terphenyl ( $\mathrm{pTp}$ ) deposited quartz plates and proposed them to replace the current HE scintillators. The R\&D studies consist of the material selection, simulation, and data collection. Details of these studies and results are described in this thesis. 


\section{CHAPTER 2 \\ THE LARGE HADRON COLLIDER AND THE COMPACT MUON SOLENOID EXPERIMENT}

\subsection{The Large Hadron Collider}

The Large Hadron Collider (LHC) [1] is the world's largest proton-proton (pp) collider and is built by the European Organization for Nuclear Research (CERN), in Geneva, Switzerland. The LHC is designed to operate with $\sqrt{\mathrm{s}}=14 \mathrm{TeV}$ to explore physics beyond the Standard Model $(\mathrm{SM})$ but it currently operates with $\sqrt{\mathrm{s}}=7$ $\mathrm{TeV}$ center of mass energy. The first $\sqrt{\mathrm{s}}=7 \mathrm{TeV}$ pp collisions at the LHC occurred in March 2010 and the collision energy is expected to be increased after the 2013 long shutdown. The LHC also provides heavy ion collisions to study the quark-gluon plasma state of the matter.

There are four different experiments at LHC; A Toroidal LHC Apparatus (ATLAS) [3], Compact Muon Solenoid (CMS), LHC-b [4], and A Large Ion Collider (ALICE) [5]. ATLAS and CMS are the two general purpose detectors designed to discover the Higgs boson and search for new physics. LHC-b is studying b quark physics (by looking for B-mesons produced in pp collisions) and CP violation to understand why matter dominates antimatter in the universe. ALICE is studying quark and gluon plasma created with heavy ion collisions to understand confinement and chiral-symmetry restoration in QCD [5]. These experiments are located at different places along the LHC ring. The schematic view of LHC and the four detectors are shown in Figure 2.1. 


\section{CERN Accelerator Complex}

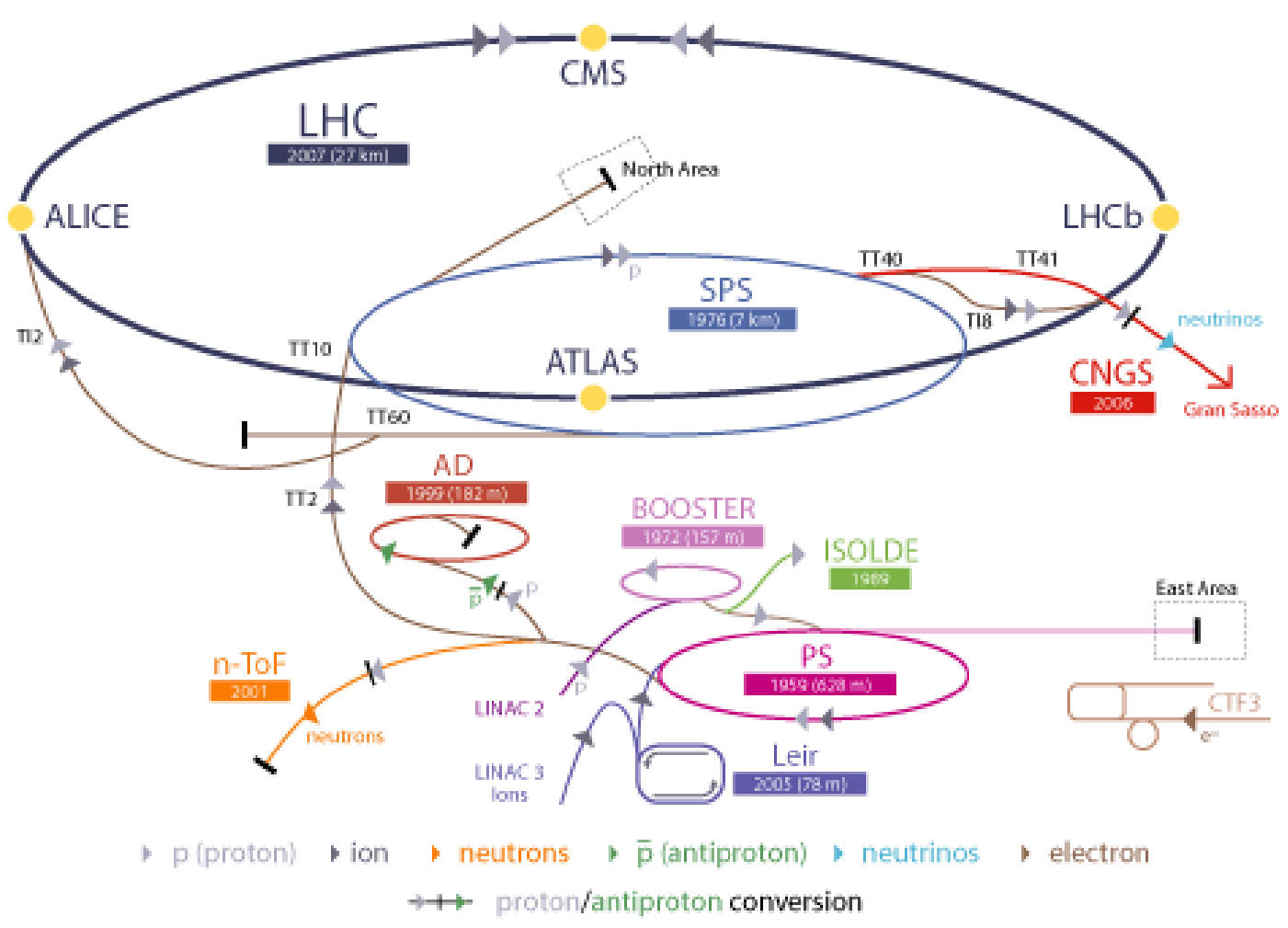

LHC Large Hadron Collider SPS Super Proton Synchrotron PS Proton Synchrotron

CNGS Cern Neutrinos to Gran Sasso ISOLDE Isotope Separator OnLine DEvice

LEIR Low Energy lon Ring LINAC LINear ACcelerator n-ToF Neutrons Time Of Flight

Figure 2.1: A schematic view of the CERN accelerator complex including the LHC, and LHC experiments [6]. 


\subsection{The Compact Muon Solenoid Experiment}

The main physics goals of the CMS experiment are to observe the Higgs particle (a hypothetical particle responsible from electroweak symmetry breaking mechanism), and discover new physics beyond the Standard Model (SM). The CMS experiment has a wide range of capabilities to look for physics processes, among which are various decays of the SM and beyond the SM (BSM) Higgs bosons, inclusive b-production, top quark production, quantum chromodynamics (QCD), diffraction, electroweak physics (W and Z), supersymmetry, extra dimensions, and technicolor.

The design of the CMS experiment is given in detail in several documents $[2$, 7-10], but here I give a brief summary based on the given references.

The Compact Muon Solenoid (Fig. 2.2) is a large detector with a $21.6 \mathrm{~m}$ length, $15 \mathrm{~m}$ diameter, and it weighs more than 14500 tons. Its compactness come from the fact that the calorimeter and the tracker fit inside the $4 \mathrm{~T}$ solenoid magnet. CMS has four main subsystems dedicated to measure the energy of photons, electrons, muons and all the other products of proton-proton collisions: (i) magnet, (ii) muon system, (iii) tracker, and (iv) calorimeters.

The innermost layer of the CMS is a silicon-based tracker which is surrounded by the scintillating crystal electromagnetic calorimeter. The electromagnetic calorimeter itself is surrounded by a sampling hadron calorimeter. The tracker and calorimeters are inside the solenoid magnet and the large muon detectors of CMS are located outside the magnet. 


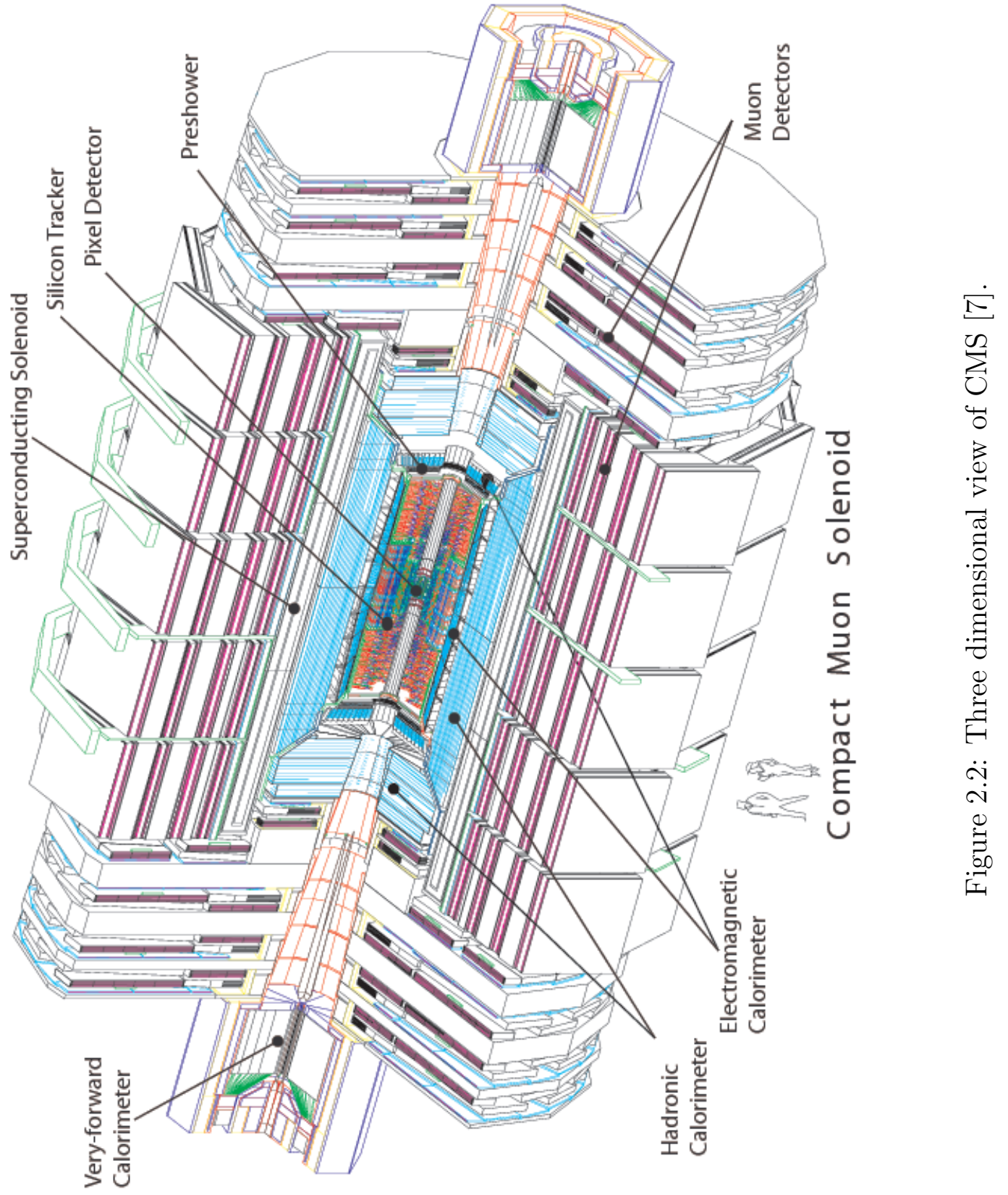




\subsubsection{The Magnet}

The CMS detector has a large superconducting solenoid magnet (Fig. 2.3) with a $13 \mathrm{~m}$ length and $6 \mathrm{~m}$ inner diameter. The bore of the magnet coil is large enough to accommodate the inner tracker and the calorimetry inside [7]. It is originally designed to produce a $4 \mathrm{~T}$ magnetic field. In order to increase the life expectancy, it was decided to operate the magnet with a $3.8 \mathrm{~T}$ magnetic field that provides a 12 Tm bending power. The bending power allows the measurement of the charge/mass ratio for the particles that leave a curved track in the magnetic field. The magnet's length/radius ratio and the high magnetic field provide a good momentum resolution for the whole detector including the forward region.

\subsubsection{The Muon System}

Reconstructing muons, measuring their momentum with a high accuracy and using them for trigger information are crucial for CMS. Since the final states with muons are important signatures for new physics such as the supersymmetry searches with three muon final states and searches for the Higgs, measuring muons with high accuracy is especially important for discovery studies. That is one of the main reasons to design CMS with a muon detector which can give a clean signal for the long lived muons.

The muon detector is the outermost layer of the CMS detector. The muon systems cover both the calorimeters and the superconducting magnet. The pseudorapidity up to $|\eta|=1.2$ in the barrel region and $0.9<|\eta|<2.4$ in the endcap regions 


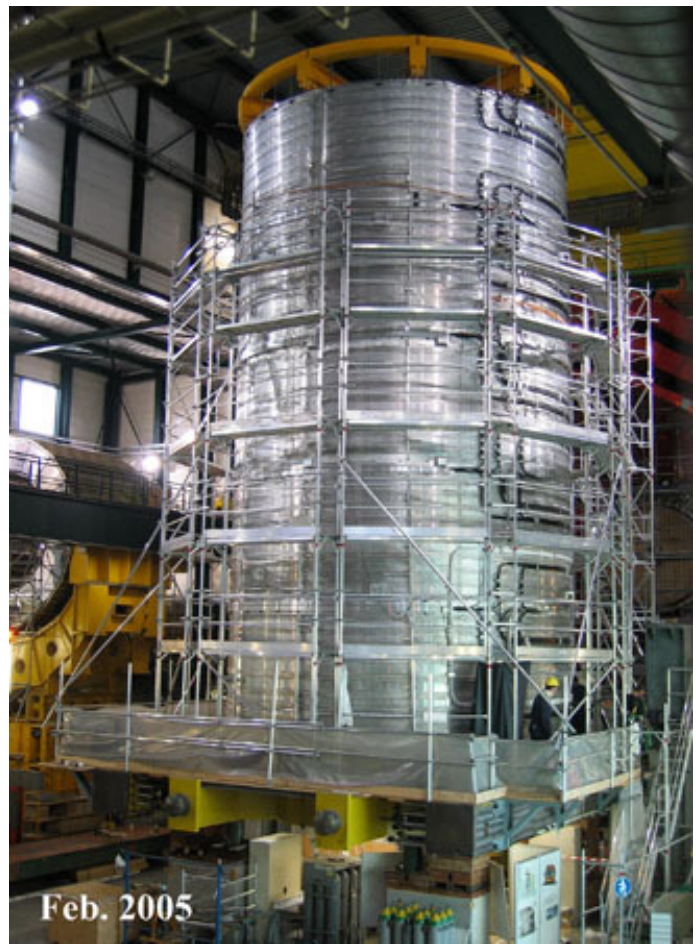

Figure 2.3: Coil of the superconducting magnet [2]. 
are covered by the four layers of muon chambers.

Three different detector technologies are used in the muon system to identify and measure muons in the whole detector. In the barrel region $(|\eta|<1.2)$, where the neutron induced background is small, the muon rate and the residual magnetic field is low, drift tube (DT) chambers are used. The cathode strip chamber (CSC) technology is used in two endcap regions $(|\eta|<2.4)$, where the magnetic field, the muon rate and the neutron induced background rate are high. As the last part of the muon system resistive plate chambers (RPC) are used both in the barrel and endcap regions.

The one quarter layout of the CMS muon system is shown in Figure 2.4. In the muon system there are a total of 1400 muon chambers with 250 DTs, 540 CSCs and 610 RPCs. The DTs and CSCs track the position of the particles and provide the trigger information while RPCs form a trigger system to decide what should be kept from the collected muon data.

\subsubsection{The Tracker}

The CMS Silicon Strip Detector is a very large tracker with total $5.4 \mathrm{~m}$ length and $1.1 \mathrm{~m}$ outer radius. The layout of CMS tracker is shown in Figure 2.6. The CMS tracking system is composed of a pixel detector and a silicon strip tracker which are designed to measure charged particle trajectories with high efficiency and provide precise reconstruction of secondary vertices originating from LHC collisions [2].

The pixel detector (Fig. 2.7) has 3 barrel layers and 2 endcap disks on each 


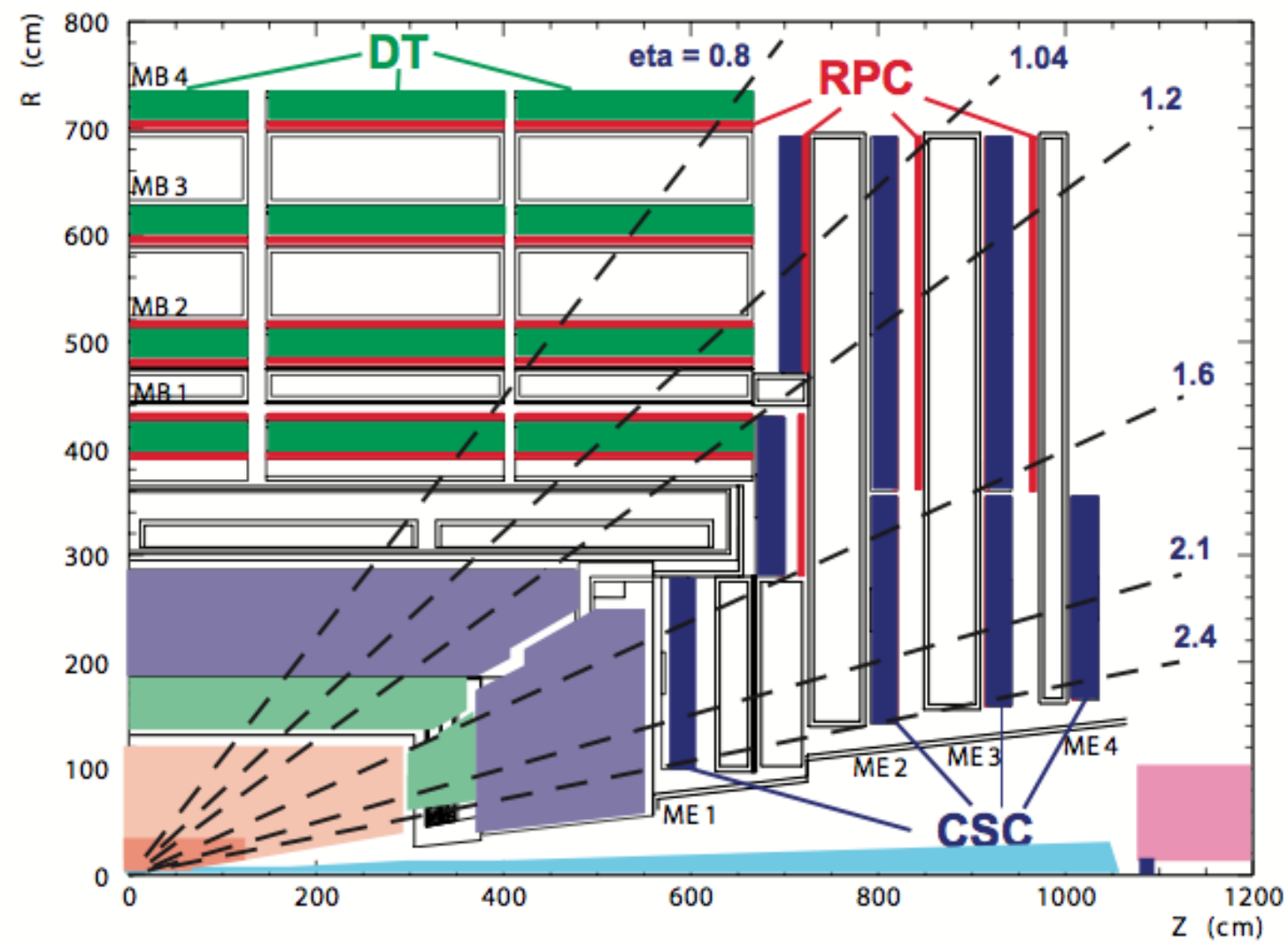

Figure 2.4: The one quarter layout of the CMS muon system [7]. 


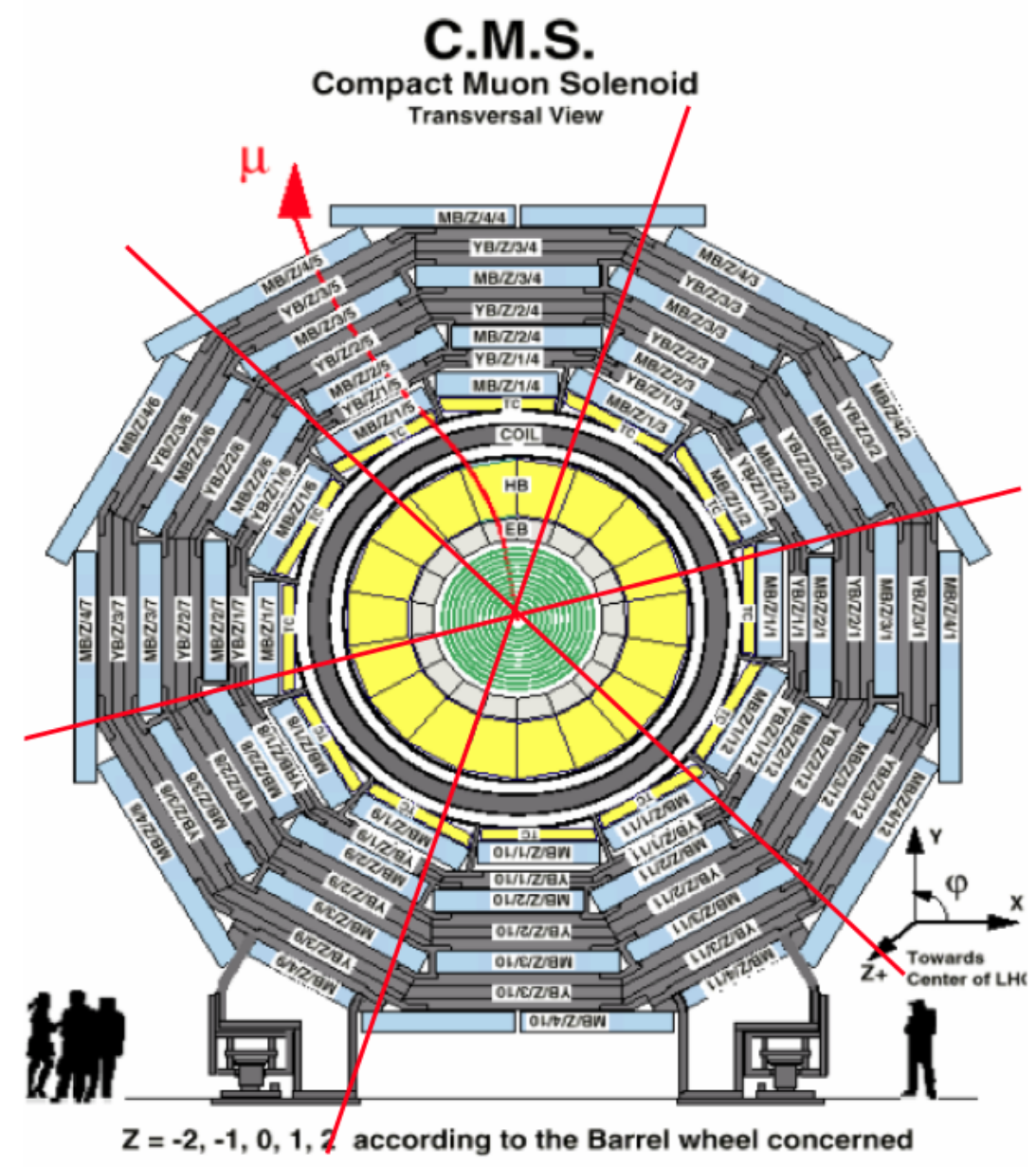

Figure 2.5: Transverse view of the Barrel Muon detector [7].

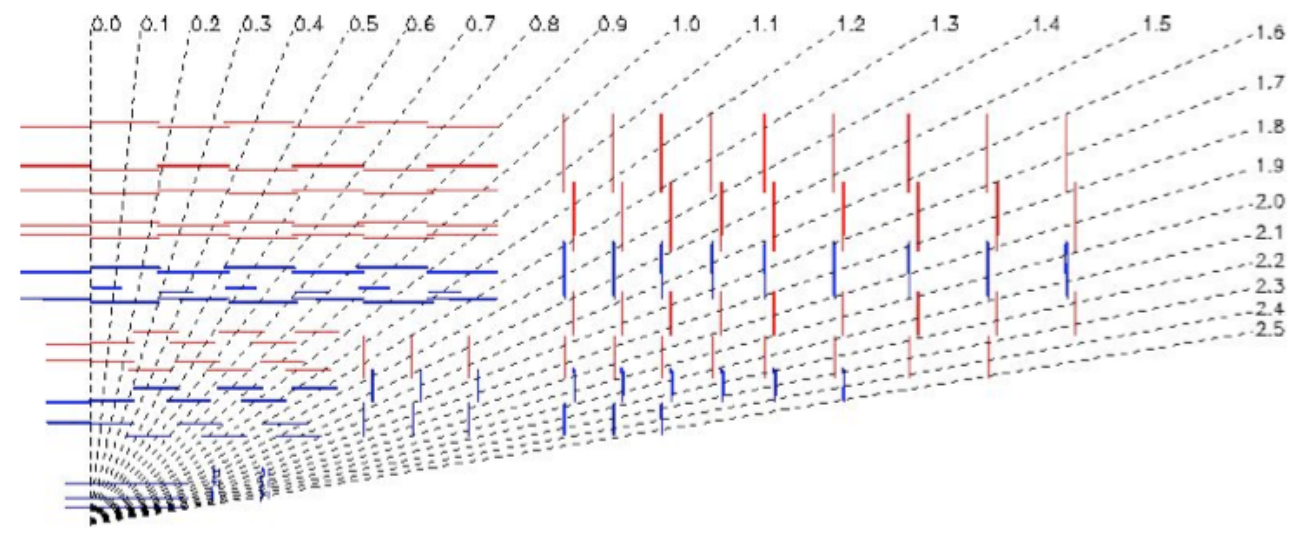

Figure 2.6: The tracker layout (1/4 of the $\mathrm{z}$ view) [7]. 
side of the barrel layers. The mean radii for the barrel layers are $4.4 \mathrm{~cm}, 7.3 \mathrm{~cm}$, and $10.2 \mathrm{~cm}$. The length of the pixel detector is $53 \mathrm{~cm}$. It provides space point information for charged particles with a very high resolution.

The silicon strip tracker is divided into two parts. The inner part consists of four barrel layers and three forward disks, whereas the outer detector consists of six barrel layers and nine forward disks. Operating together with the pixel detector, the silicon strip detector is used to reconstruct tracks and measure momentum for all tracks above $1 \mathrm{GeV}$.

The tracking efficiency for high $\mathrm{p}_{\mathrm{T}}$ tracks is better than $98 \%$ in the pseudorapidity range $|\eta|<2.5$. For the pseudorapidity range $|\eta|<1.6$, the transverse momentum resolution for high $\mathrm{p}_{\mathrm{T}}$ tracks $\left(\mathrm{p}_{\mathrm{T}}>100 \mathrm{GeV}\right)$ is $1-2 \%$. The CMS tracker reconstruction efficiency for single muons is about $99 \%$ for the large $\eta$ region. Due to gaps between the ladders of the pixel detector, it slightly decreases for $|\eta| \approx 0$ and drops drastically for the high $\eta$ region due to the reduced coverage of the pixel forward disks. The efficiency is generally lower for pions and hadrons because of the interactions with the material in the tracker [2].

\subsubsection{The Calorimeters}

The CMS calorimeters are used to measure the energy of electrons, photons and jets with a high precision. The hermetic coverage also provides a high accuracy measurement for the missing transverse energy, which is an important quantity for new physics searches. The CMS calorimeter consists of electromagnetic and hadronic 


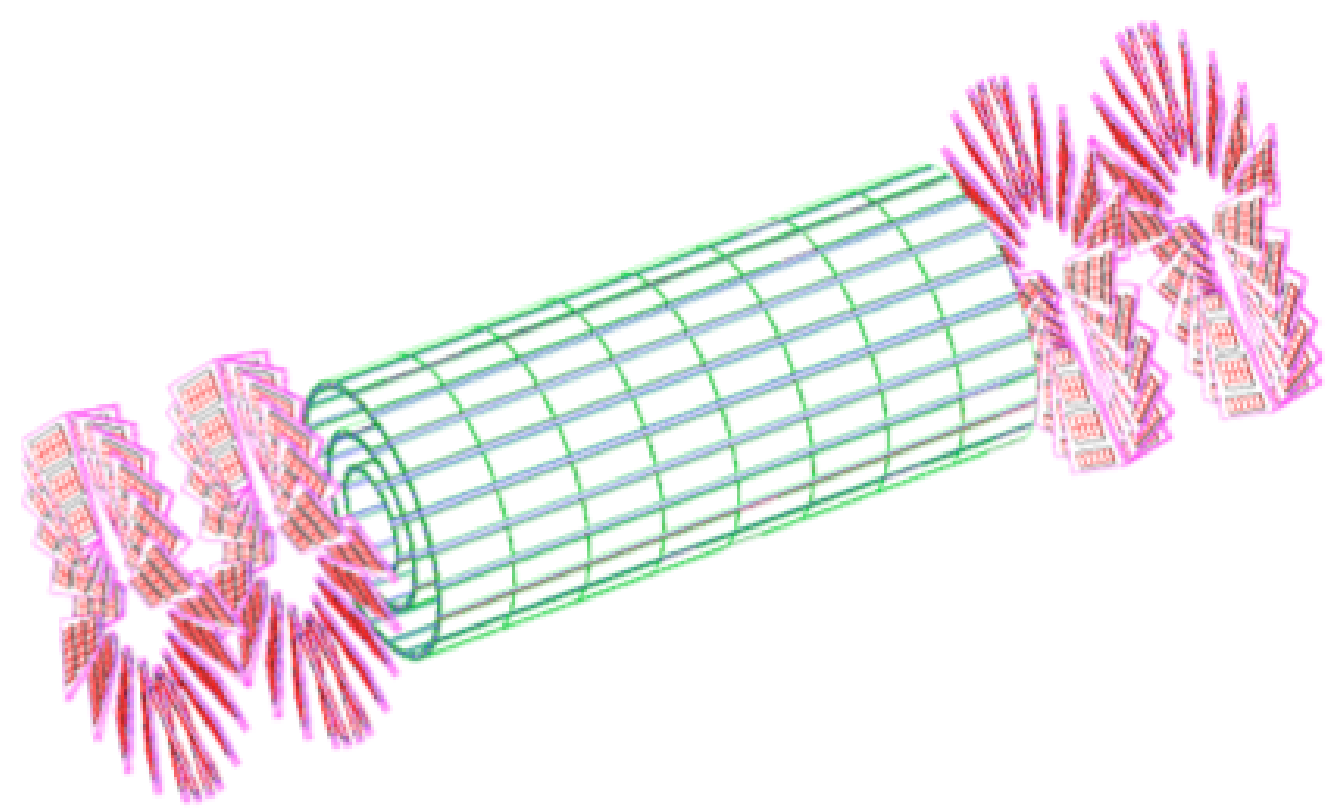

Figure 2.7: Layout of pixel detectors in the CMS tracker [7].

components.

The electromagnetic calorimeter (ECAL) (Fig. 2.2) is made up of a barrel and two endcap sections. It forms a layer between the tracker and the hadronic calorimeter (HCAL). It is made of 61200 high density lead tungstate $\left(\mathrm{PbWO}_{4}\right)$ crystals, which allows the calorimeter to be fast and radiation resistant. The main design purpose of ECAL is to detect the decay of the Higgs boson to two photons, which will be achieved by its excellent energy resolution provided by the homogeneous and fast response crystals.

The HCAL calorimeter (Fig. 2.2) consists of barrel (HB), endcap (HE), and forward $(\mathrm{HF})$ parts. The barrel and endcap parts are sampling calorimeters that 
surround the ECAL and the tracking system and cover the pseudorapidity range of $|\eta|<3.0$. The forward part extends this coverage up to $|\eta|<5$, and it consists of steel absorbers with quartz fibers embedded in it. The HCAL measures energy, position and the arrival time of interacting particles and provides an indirect measurement of non-interacting particles (such as neutrons and neutrino). 


\section{CHAPTER 3 \\ R\&D STUDIES FOR HE UPGRADE AT CMS}

\subsection{Introduction}

The Large Hadron Collider (LHC) started to provide collisions in November 2009 with $450 \mathrm{GeV}$ energy per proton beam. The first $7 \mathrm{TeV}$ (center of mass energy) collisions took place on March 30, 2010 with an instantaneous luminosity of $\sim 2 \times 10^{27}$ $\mathrm{cm}^{-2} \mathrm{~s}^{-1}$. The LHC luminosity improved in time and reached up to $\sim 2 \times 10^{32}$ until the extended technical stop in December 2010. The total integrated luminosity recorded in the CMS detector for the 2010 run period is shown in Figure 3.1. In February 2011, the LHC restarted and will continue running until the end of 2012 with a short technical stop at the end of 2011. It is expected to collect $\mathcal{O}(10) \mathrm{fb}^{-1}$ of data during this run period. It is planned that at the end of 2012, the LHC will have a long shutdown to be ready for running at the design luminosity and energy.

If the LHC experiments discover the Higgs boson or new physics, the current luminosity will be enough to study its mass and some of the expected branching ratios but not much of its other properties. To study rare events such as MSSM Higgs and Higgs coupling to itself, we will need a higher number of events, and this can only be achieved by the higher luminosity conditions. The LHC currently operates with the peak luminosity $\sim 2 \times 10^{33} \mathrm{~cm}^{-2} \mathrm{~s}^{-1}$. This luminosity will be improved in time and is planned to be up to $\sim 10 \times 10^{34} \mathrm{~cm}^{-2} \mathrm{~s}^{-1}$ by 2023 .

The luminosity upgrade plans will be completed in two phases. In the first 


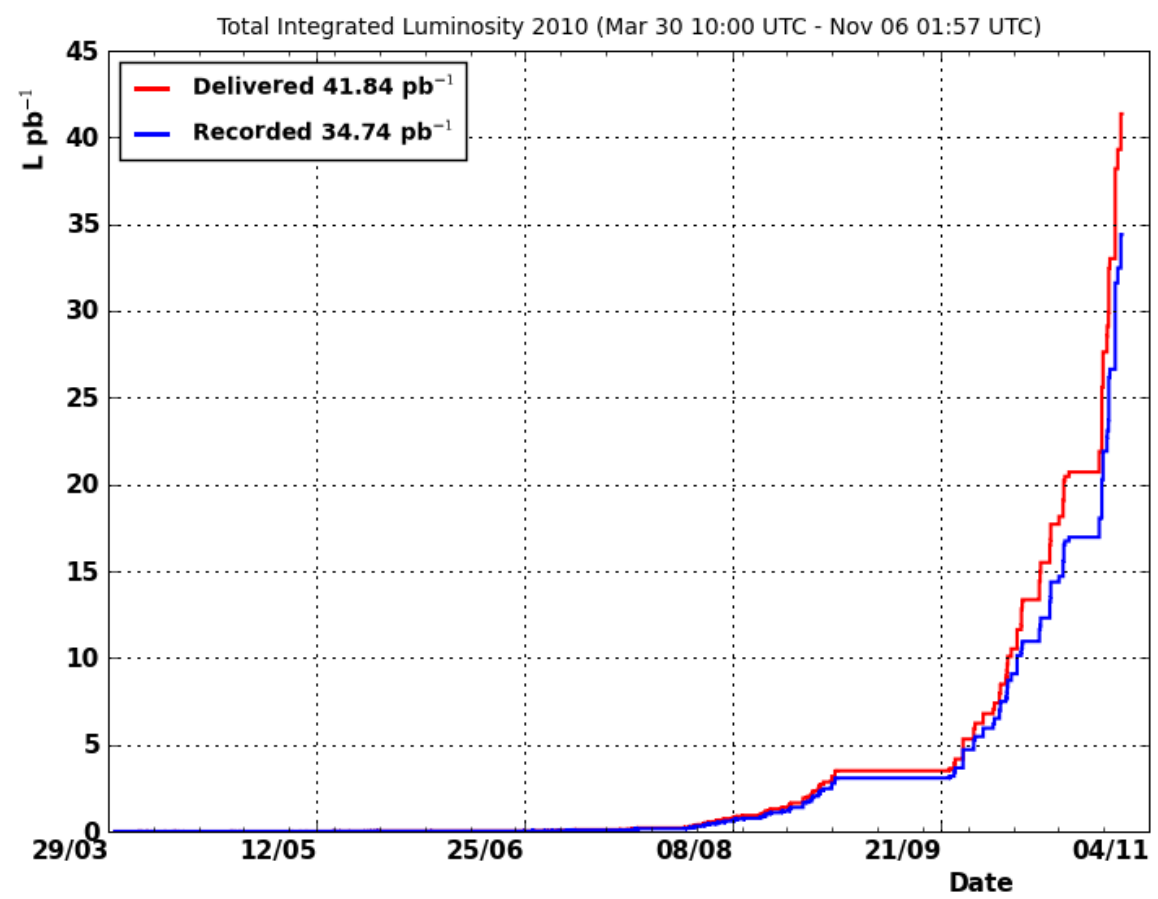

Figure 3.1: Total integrated luminosity recorded in CMS during 2010 [11]. 
phase, no detector upgrade will be necessary and the peak luminosity will be increased to $\sim 2 \times 10^{34} \mathrm{~cm}^{-2} \mathrm{~s}^{-1}$. For the second upgrade phase, the aim is to reach $\sim 10 \times 10^{34}$ $\mathrm{cm}^{-2} \mathrm{~S}^{-1}$ and for that increase it will be necessary to upgrade detectors and the LHC injector.

Before the second phase, the accumulated radiation will damage CMS and the other detectors. The damage caused by the radiation will be severe in the higher pseudorapidity ranges. As a result, the active parts of the CMS detector will lose their efficiencies and eventually stop providing light collection. The scintillator tiles used in the CMS Hadronic Endcap (HE) Calorimeters are one of those parts. To solve this radiation problem for higher luminosity run conditions, we proposed to replace the current HE scintillators with p-terphenyl (pTp) deposited on quartz plates.

In this chapter, the HE calorimeter is introduced, and our R\&D studies based on the proposed quartz plates are presented.

\subsection{Hadronic Endcap Calorimeter and Radiation Damage}

The CMS Hadronic Endcap (Fig. 3.2) together with other components in the calorimeter, is used for jet energy and missing transverse energy measurements, and covers the pseudorapidity of $1.3<|\eta|<3.0$. The current design of the CMS HE calorimeter consists of 19 layers of plastic scintillator tiles which are placed between $78 \mathrm{~mm}$ thick brass absorber plates. The thickness of the scintillators is $3.8 \mathrm{~mm}$. Light produced in the plastic scintillators (Kuraray SCSN81) is carried to hybrid photodiode (HPD) photodetectors by double clad wavelength shifter (WLS) fibers (Kuraray Y- 
11). Scintillators and WLS fibers have been shown to be moderately radiation hard, up to 25 kGy [10]. With increasing luminosity, both scintillator tiles and WSF are expected to face higher radiation problems, and this will cause a severe reduction in the light yield. It will become necessary to replace the current HE scintillators.
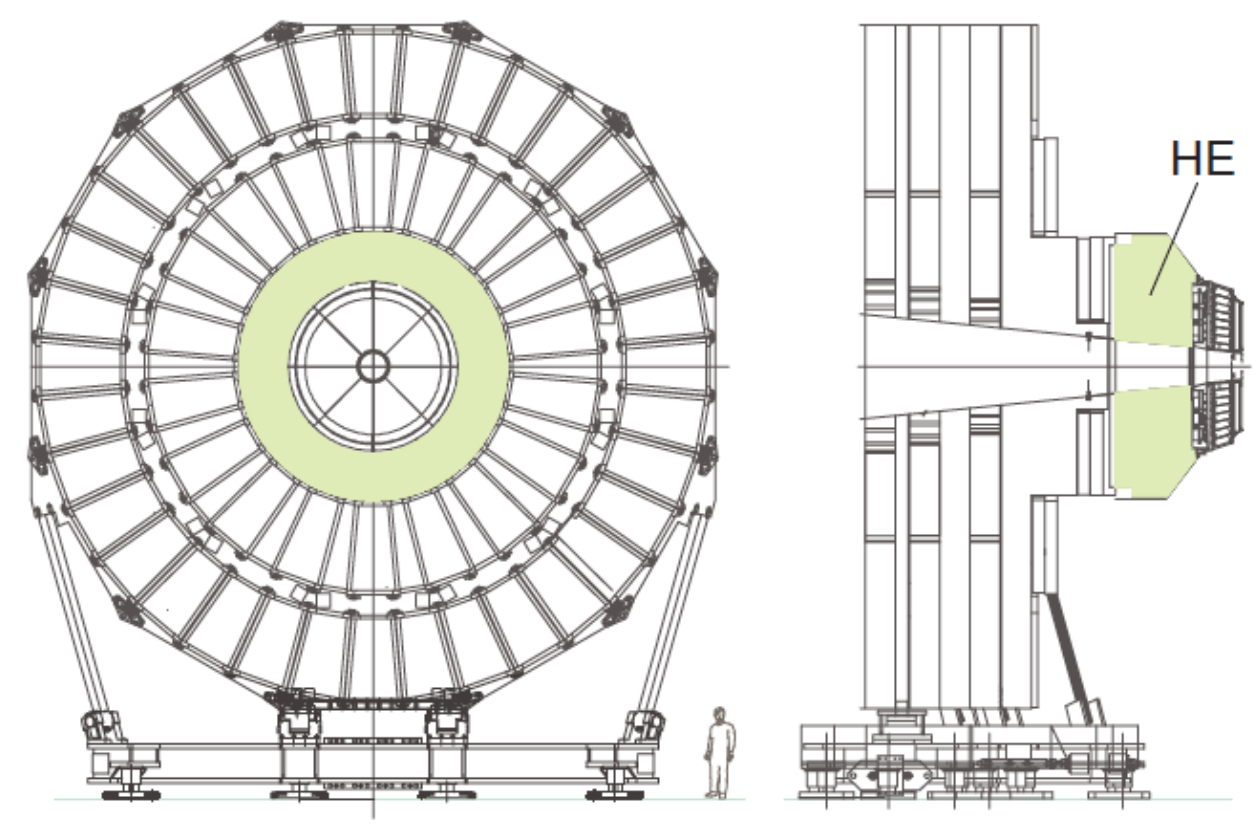

Figure 3.2: Hadron endcap calorimeter mounted on the endcap iron yoke [2].

We propose to replace the original scintillator tiles with quartz plates. Previous studies have shown that quartz plates are radiation hard up to 12.5 MGy [12-14]. One disadvantage of using quartz plates is the source of light production, photons from the C̆erenkov process, which creates acutely fewer photons than the scintillation 
process. Since light collection created with the Cerenkov process increases with $1 / \lambda^{2}$ we can collect more photons if we use a wavelength shifter method with UV absorption spectra. For this purpose different wavelength shifters, including p-terphenyl (pTp), $4 \%$ gallium doped zinc oxide (ZnO:Ga), o-terphenyl (oTp), m-terphenyl (mTp) and p-quarterphenyl (pQp) are tested [15].

The proposed wavelength shifters are tested both for radiation hardness and light collection. The Indiana University Cyclotron Facility (IUCF), University of Mississippi CMS Laboratories, and CERN beam lines are used to test the candidates for different irradiation levels. To test the light collection enhancement, also a 20 layer sampling calorimeter prototype with pTp coated quartz plates is designed and used at the CERN H2 area for beam test.

\subsection{Selection of Wavelength Shifter}

To choose the best light enhancement wavelength shifter (WLS), quartz plates are coated with several different wavelength shifters: pTp, ZnO:Ga, oTp, mTp, and pQp. The proposed wavelength shifters are tested for light yield at Fermilab Meson Test Beam Facility and at the CERN H2 area with proton, pion, and electron beams. Quartz plates are prepared with various wavelength shifters which are deposited at different thicknesses. The coating process for all the proposed materials is done at the University of Iowa CMS laboratories and Fermilab Thin Film Laboratory by evaporating the WLS in a vacuum chamber (Fig. 3.3) and applying to the quartz, except for $\mathrm{ZnO}$ :Ga. For $\mathrm{ZnO}$ :Ga, because of its molecular properties, radio frequency 

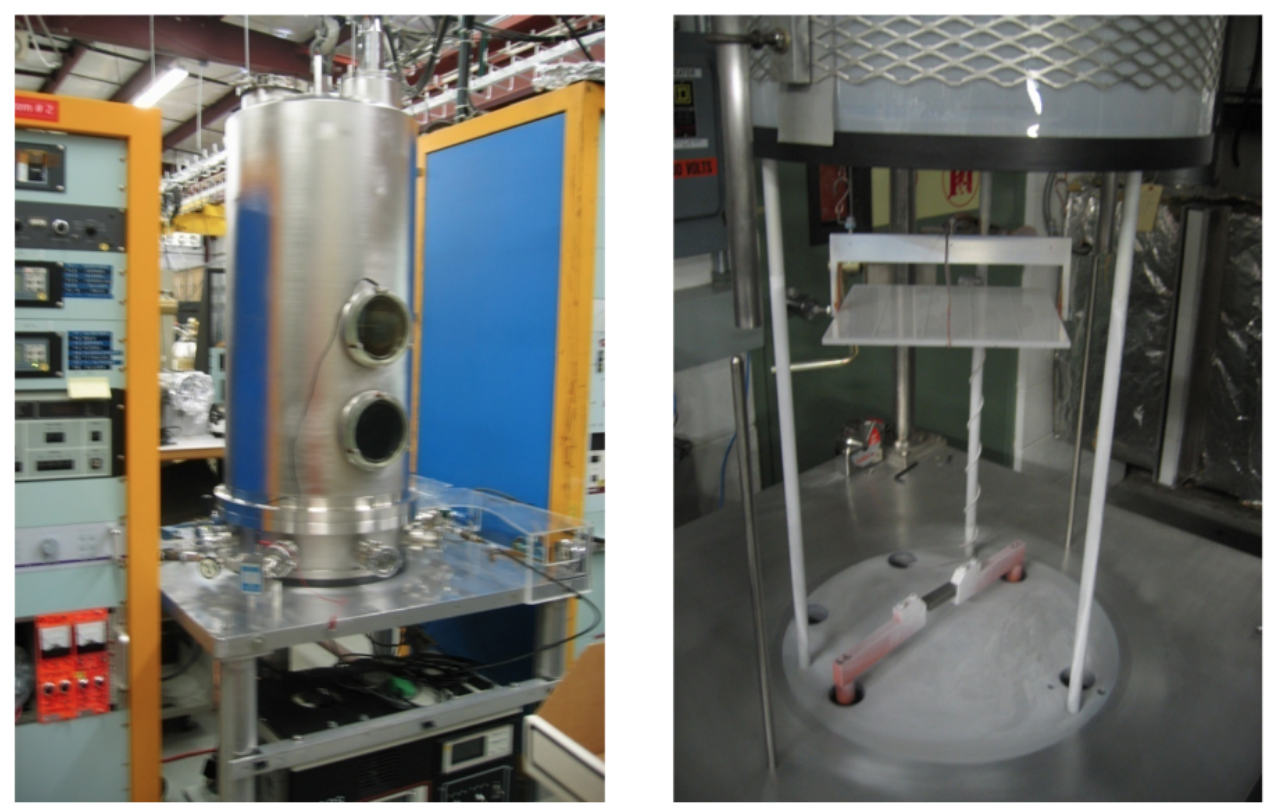

Figure 3.3: Fermilab Thin Film Laboratory vacuum chamber (pTp evaporating system).

(RF) sputtering (Fig. 3.4) is used.

The light collection is performed by Hamamatsu R7525-HA photomultiplier tubes (PMTs) [16-18], from the edge of the plates. Test results show that the one side coated quartz plates with $2 \mu \mathrm{m}$ thickness of pTp and $0.2 \mu \mathrm{m}$ of $\mathrm{ZnO}$ :Ga perform the best light collection for various energy ranges. The comparison of the single photoelectron signal distribution for $2 \mu \mathrm{m}$ pTp and $0.2 \mu \mathrm{m}$ of $\mathrm{ZnO}$ :Ga deposited plates to plain quartz plate is shown in Figure 3.5. The setup that is used at the Fermilab Meson Test Beam Facility to test the light output from single quartz plate (WLS deposited) is shown in Figure 3.6. The test beam results show both pTp and $\mathrm{ZnO}$ :Ga deposited quartz plates increase the light yield by at least a factor of 


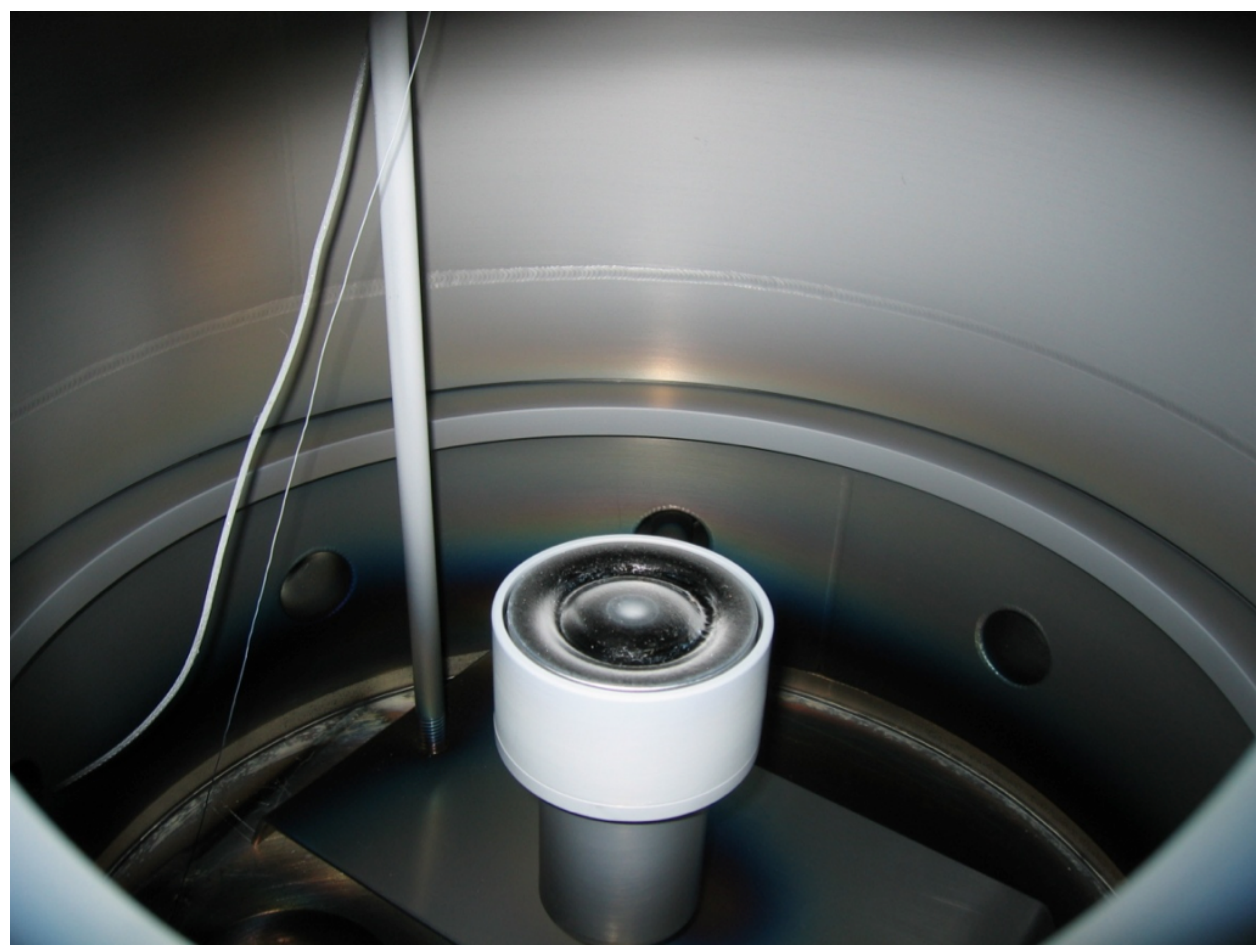

Figure 3.4: Fermilab Thin Film Labarotary ZnO:Ga sputtering system and guns. 
four. Since ZnO:Ga does not show any further light enhancement and the deposition process is more expensive we decided to focus on $\mathrm{pTp}$ deposited quartz plates for our R\&D studies.

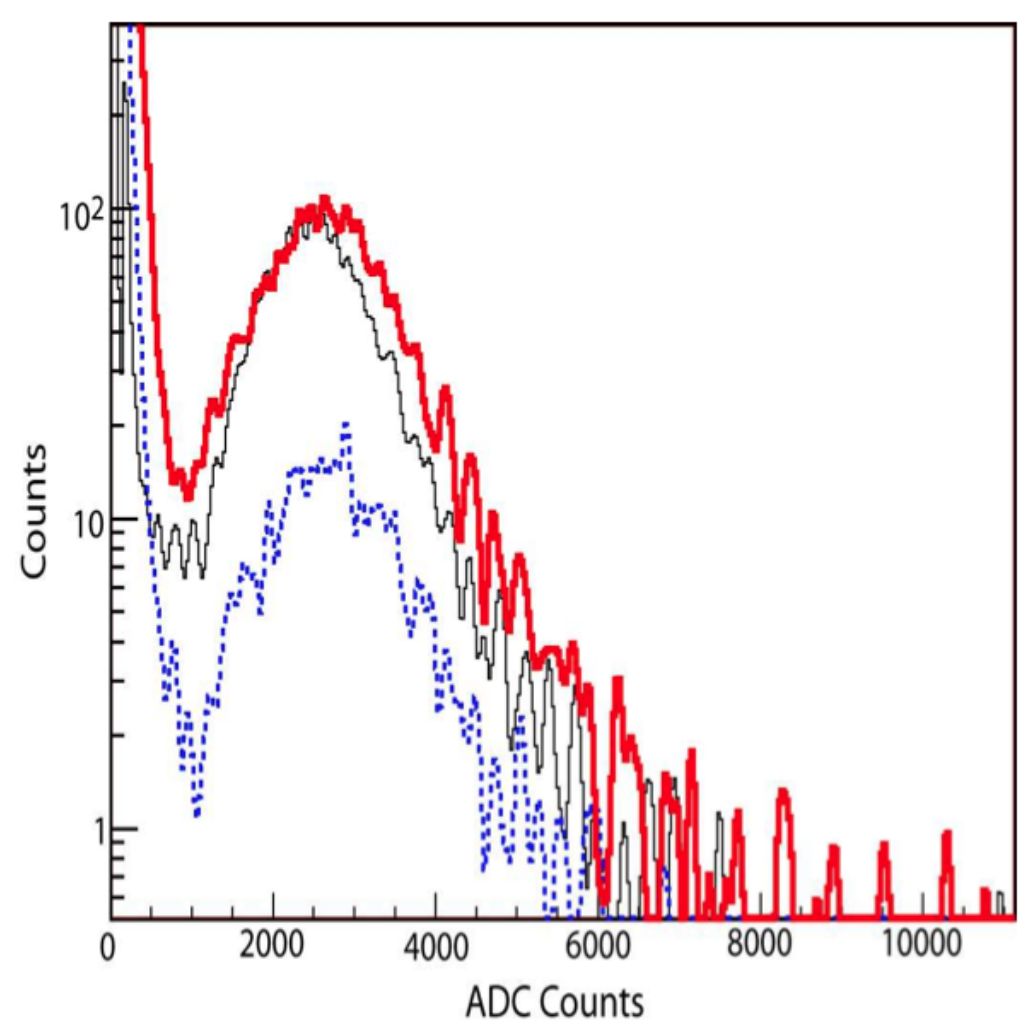

Figure 3.5: The comparison of single photoelectron level signal distributions from $2 \mu \mathrm{m}$ thickness of pTp (red solid line), and $0.2 \mu \mathrm{m}$ of $\mathrm{ZnO}$ :Ga (black solid line) deposited plates to the plain quartz plate (dashed blue line) [15].

The pTp as a fiber coating is also tested for its radiation hardness. The tests are performed with the proton beam at the IUCF and the CERN beam lines and 


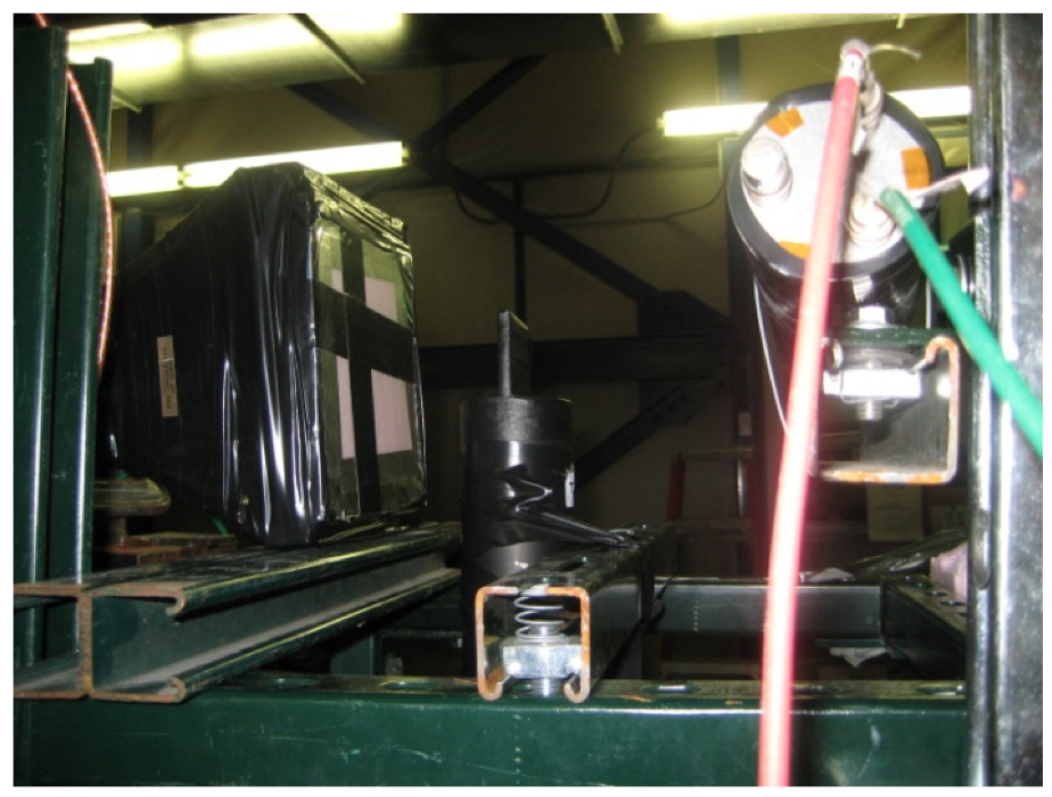

Figure 3.6: The WLS deposited quartz plate light output test setup.

with activated ${ }^{90} \mathrm{Sr}$ at University of Mississippi CMS Laboratories. The light output of pTp samples are compared before and after irradiation. Figure 3.7 shows results of different proton irradiation levels. Studies show that after $200 \mathrm{kGy}$, pTp loses $16 \%$ of the initial light output and the radiation damage rate slows down. After $400 \mathrm{kGy}$ we still have more than $80 \%$ of our initial light production.

\subsection{Calorimetric Properties of Coated Quartz Plates}

The studies summarized in Section 3.3 show that the best candidate quartz plate to replace scintillator tiles in $\mathrm{HE}$ is the one with one side deposited with 2 $\mu \mathrm{m}$ pTp. To test the detector capability of those plates we designed a calorimeter prototype that consists of 20 layers of quartz plates with $7 \mathrm{~cm}$ iron absorbers between 


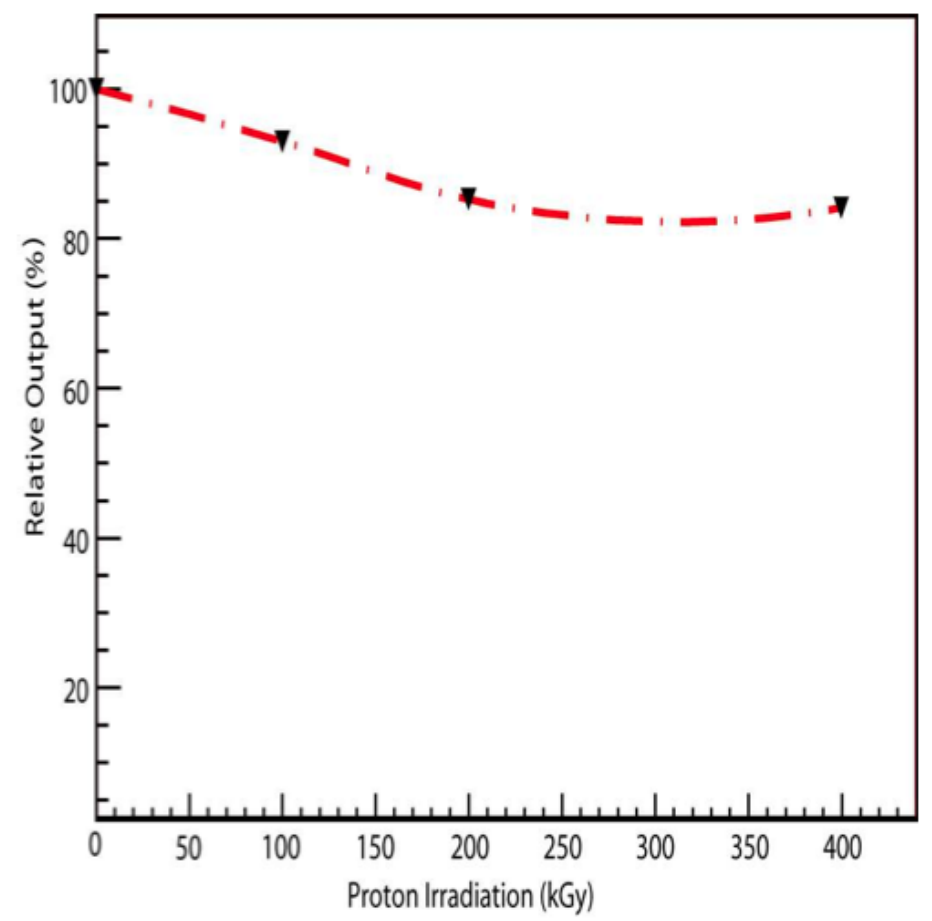

Figure 3.7: Light output from pTp sample after proton irradiation versus proton irradiation level with simple fitted line [15]. 
each layer. One edge of each plate was polished for better PMT optical coupling. The C̆erenkov light generated in the pTp deposited quartz plates was read out by Hamamatsu R7525-HA PMTs from this polished edge.

In order to have flexibility for changing absorber thickness between each plate, the plates were prepared as stand-alone units with PMTs attached to them. Each plate was wrapped with aluminized mylar for good reflectivity and then with Dupont Tyvek for having a light tight setup.

The prototype was tested for its hadronic and electromagnetic shower measurement capabilities at the CERN H2 area. For the hadronic configuration $7 \mathrm{~cm}$ iron absorbers were placed between each individual quartz plates, and the prototype was tested with $30,50,80,130,200,250,300$, and $350 \mathrm{GeV} \pi^{-}$beams. In the electromagnetic configuration (Fig. 3.8) $7 \mathrm{~cm}$ iron absorbers were replaced by $2 \mathrm{~cm}$ absorbers and the configuration was tested with 50, 80, 100, 120, 150 and $175 \mathrm{GeV}$ energy electron beams. The setup was also simulated by using GEANT4 [19,20] with the LHEP physics package.

\subsection{Results}

Figure 3.9 shows the longitudinal shower profile for various $\pi^{-}$energies. Simulation results (solid lines) along with data points are shown together for the corresponding energies. The charge distribution of the calorimeter prototype for 300 $\mathrm{GeV} \pi^{-}$with hadronic configuration is shown in Figure 3.10.

Figure 3.11(a) shows detector linearity tests for hadronic calorimeter setup 


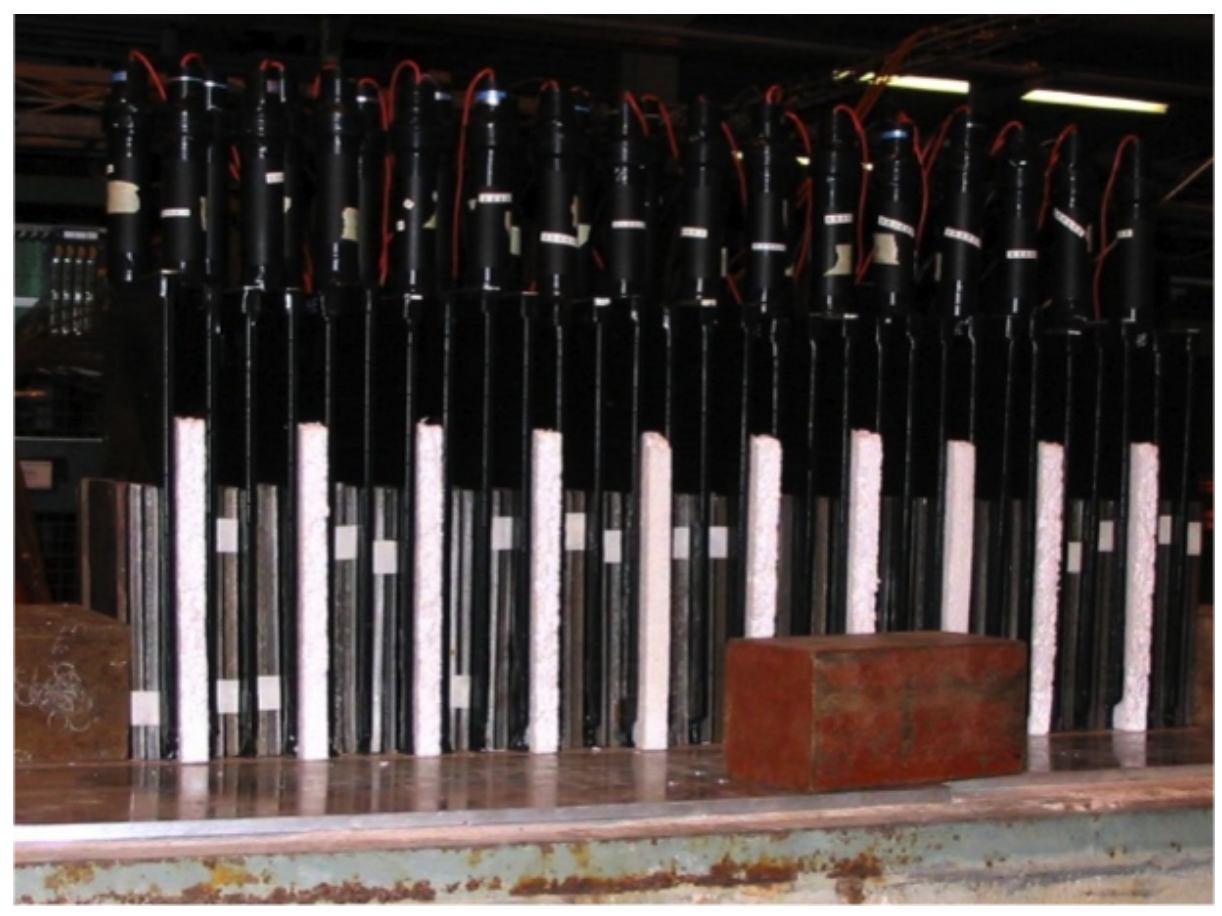

Figure 3.8: The calorimeter prototype in the electromagnetic configuration.

with various $\pi^{-}$beam energies where data points are shown by red circles and simulation points are illustrated by the blue squares. Solid line represents the fit to the data, and the dashed line to the simulation points. Data and simulation results are generally in good agreement. The discrepancies seen in the fits are due to limited statistics in the data. Figure 3.11(b) shows data and simulation results for the hadronic calorimeter energy resolution. Both data and simulations are fitted to the non-compensated energy resolution parametrized as

$$
\frac{\sigma(E)}{E}=\frac{A}{\sqrt{E}} \oplus \frac{B}{E} \oplus C
$$

where $\mathrm{A}$ is the stochastic term, $\mathrm{B}$ is the noise, and $\mathrm{C}$ is the constant term [21]. With a 


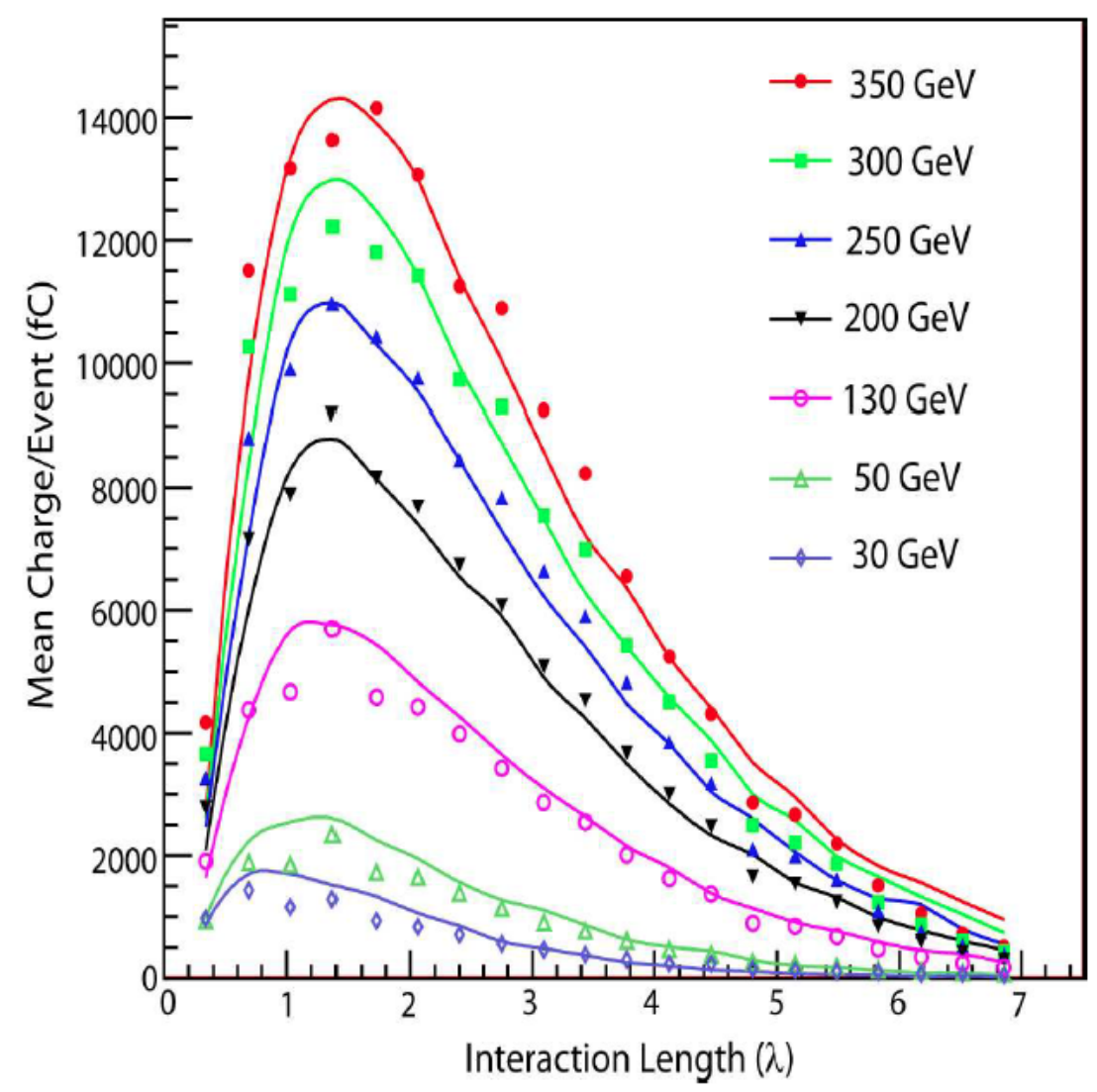

Figure 3.9: Longitudinal shower profile for different pion energies. Simulations are shown with solid line whereas data is represented by the points [15].

negligible noise term, both data and simulation show similar energy resolution, which can be described as

$$
\frac{\sigma(E)}{E}=\frac{210.3 \%}{\sqrt{E}} \oplus 8.8 \%
$$

Although the main purpose of this study was to find a solution to radiation hardness problem of the CMS HE detector, we also tested the radiation hard electromagnetic (em) configuration. The test setup is the same as the hadronic configuration 


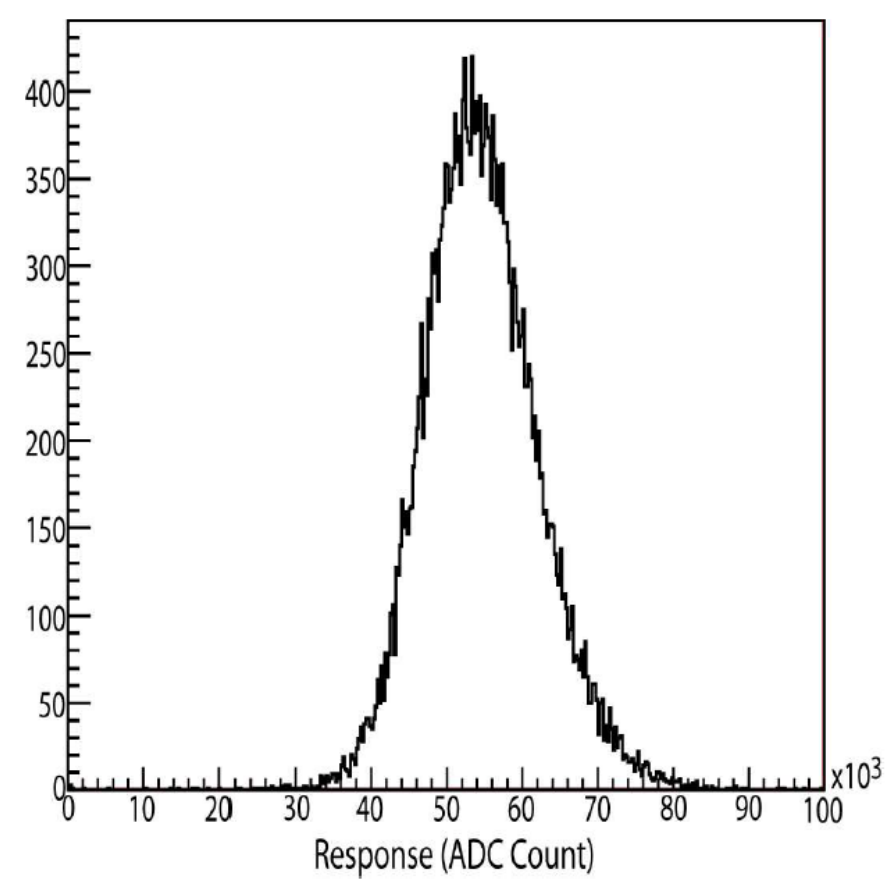

Figure 3.10: $300 \mathrm{GeV}$ pion response of pTp deposited quartz plate calorimeter [15].

except for the absorber thickness.

The longitudinal shower profile for various electron beams is shown in Fig. 3.12. The simulation and data points are in a good agreement. The charge distribution of the calorimeter prototype for the EM configuration with a $100 \mathrm{GeV}$ electron beam is shown in Figure 3.13.

The detector linearity (within 3\%) and the energy response for the EM prototype are shown in Figures 3.14(a) and 3.14(b). The data and the simulations converge to a constant value at $5.6 \%$, but below the $120 \mathrm{GeV}$ the simulations predict a better 


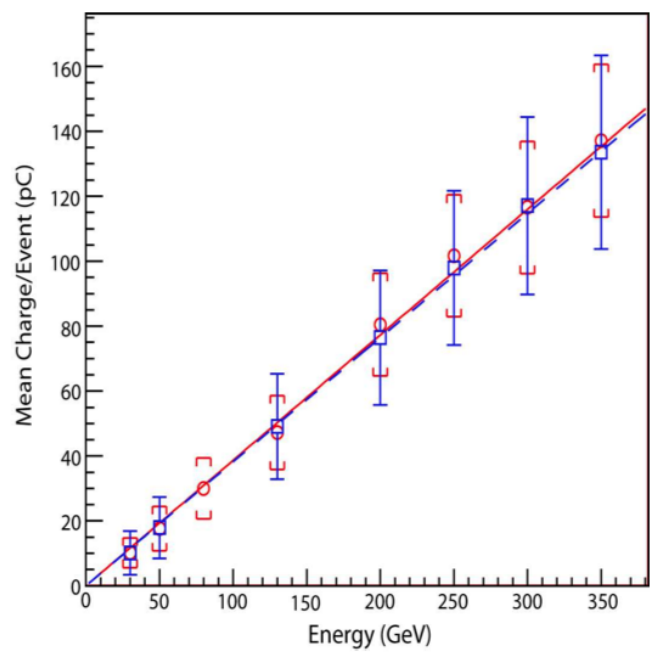

(a) Hadronic Detector Linearity

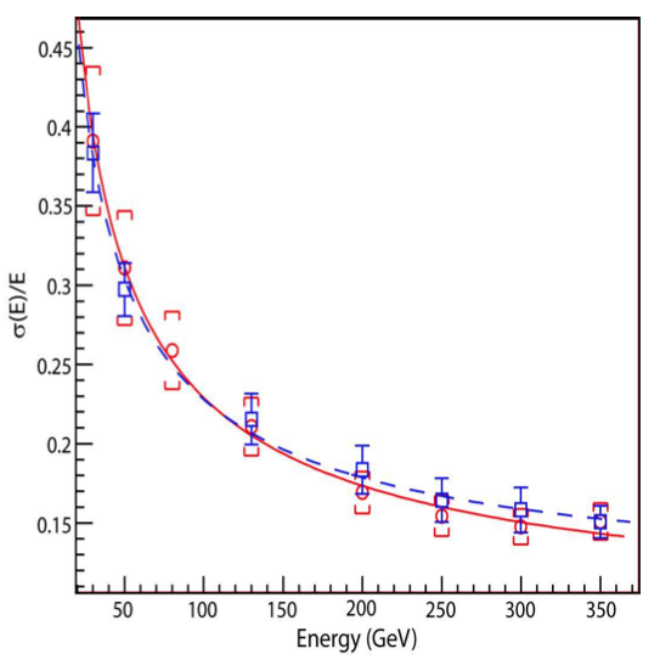

(b) Hadronic Energy Resolution

Figure 3.11: Hadronic detector linearity and energy resolution, data (red circle and solid line) and Geant4 simulations (blue square and solid line), for the calorimeter prototype [15]. 


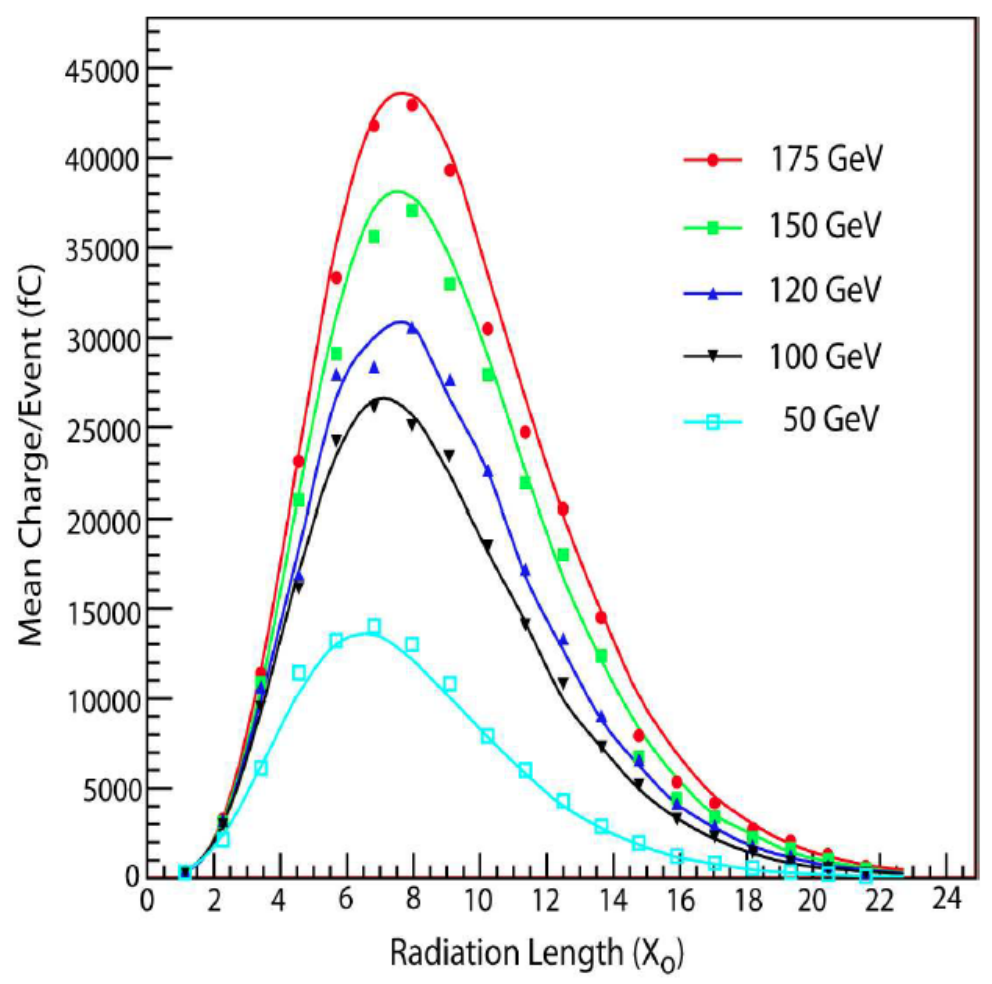

Figure 3.12: Longitudinal shower profile for different electron energies. Simulations are shown with solid line whereas data is represented by the points [15].

energy resolution. The overall energy resolution can be given as

$$
\frac{\sigma(E)}{E}=\frac{26 \%}{\sqrt{E}} \oplus \frac{4.5 \%}{E} \oplus 5.6 \%
$$

\subsection{Conclusion and Future Plans}

We proposed to use quartz plates to replace the current HE scintillator tiles during the higher luminosity upgrade phase. We showed that the quartz plate light yield can be increased with pTp deposition by at least a factor of four. Both pTp and quartz plates are radiation hard and cost efficient. 


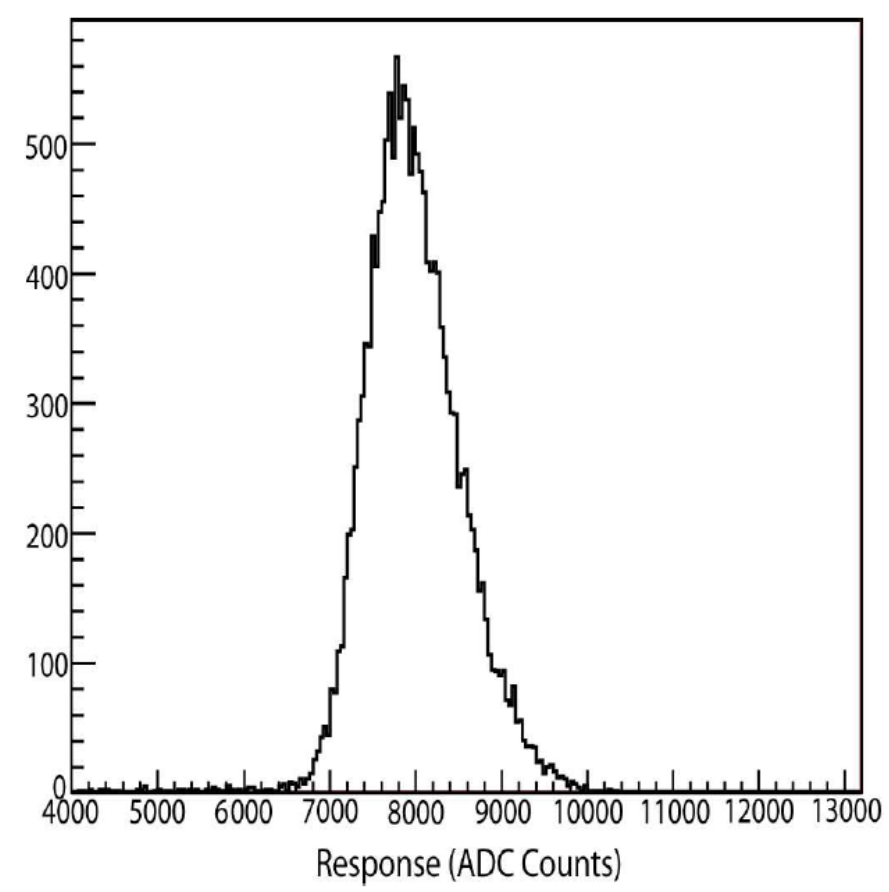

Figure 3.13: $100 \mathrm{GeV}$ electron response of the calorimeter prototype [15].

We also tested pTp deposited quartz plates for their detector capabilities in a calorimeter. The test beam results showed that a 20 layer calorimeter prototype had a $15 \%$ hadronic resolution for a $350 \mathrm{GeV}$ pion beam, which is a promising result considering the possible energy leakage from the undersized prototype. On a bigger scale the proposed calorimeter can reach up to $8 \%$ hadronic resolution, which is the current $\mathrm{HE}$ calorimeter performance for a $300 \mathrm{GeV}$ pion beam energy.

The final phase of the $R \& D$ study proposes alternative readout options for the CMS Hadronic Endcap since the current PMTs cannot be used due to the high radiation level and the design of the HE. One of those options is the use of microchannel PMT, which are fast in response, have high gain, and most importantly are 

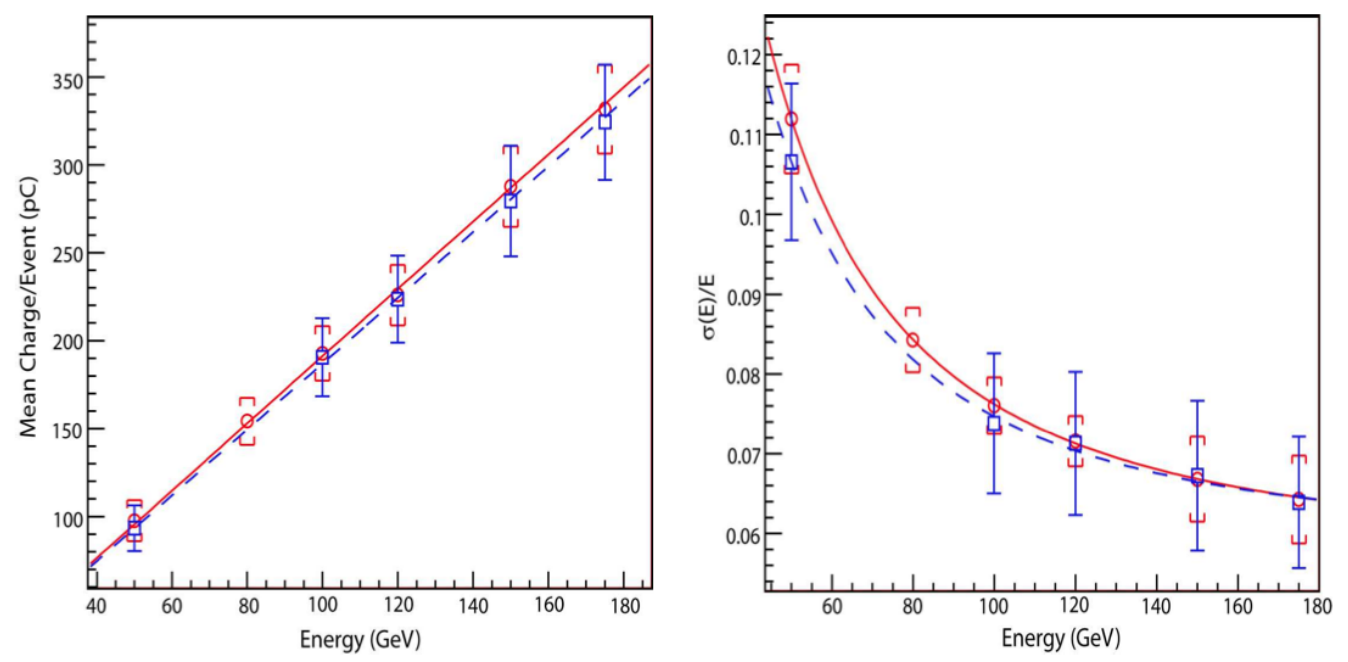

(a) Electromagnetic Detector Linearity (b) Electromagnetic Energy Resolution

Figure 3.14: Electromagnetic detector linearity and energy resolution, data (red circle and solid line) and Geant4 simulations (blue square and dashed line), for the calorimeter prototype [15]. 
radiation hard and cost less. The new readout options, as they become available, will be tested during future test beam studies and the best $\mathrm{HE}$ readout option for the super LHC run conditions will be found. 


\section{CHAPTER 4 \\ INCLUSIVE SEARCH FOR NEW PHYSICS AT CMS WITH JETS AND MISSING MOMENTUM SIGNATURE}

\section{$4.1 \quad$ Introduction}

The Standard Model (SM) is a theoretical model which describes the elementary particles and explains the fundamental interactions between them. It has been tested during the past several decades and the SM predictions are found to be highly consistent with experimental data. All the particles predicted by the SM have been discovered by experiments except the Higgs boson [22].

Although the SM can explain nature's working mechanism with a high accuracy, there are still questions it cannot answer such as:

- Why some force carriers have mass but others do not?

- Why does the SM have 19 parameters to determine particle masses and the interactions?

- How does the electroweak symmetry breaking mechanism work?

- Why does the SM have 3 generations of particles?

- What is the reason behind the observed mass spectrum of particle generations?

- Why are the strengths of forces so different from each other?

- Can gauge couplings be unified at a high mass scale?

- What is the reason behind the apparent matter-antimatter asymmetry in the universe? 
- What is the source of cold dark matter in the universe?

Many beyond the SM physics theories such as Supersymmetry (SUSY) [23], extra dimensions [24], Technicolor [25], and fourth family [26] try to address these questions. Since it can lead to incorporation of gravity to particle physics, SUSY is one of these many new models which is favored by many theorists and experimentalists.

The Minimal Supersymmetric Standard Model (MSSM) is the supersymmetric extension of the SM with minimal particle content [27-29]. The MSSM introduces a spectrum of new particles that are superpartners (sparticles) of SM particles with SM couplings. The superpartners of SM fermions are named with the prefix "s" (sfermion, slepton, squark) whereas the superparners of bosons are named with the suffix "ino" (gaugino, higgsino, wino). The supermultiplets are divided into two categories:

- chiral supermultiplets which are scalar particles and their superpartners

- gauge supermultiplets which are vector bosons and their superpartners

The SM particles and their superpartners with $\mathrm{SU}(3)_{\mathrm{C}} \times \mathrm{SU}(2)_{\mathrm{L}} \times \mathrm{U}(1)_{\mathrm{Y}}$ quantum numbers are tabulated in Table 4.1.

The sparticles have the same masses as their particle partners, however, this is only valid for the theories with unbroken symmetry. They have the same quantum numbers as elementary particles but differ from the SM particles by a half-spin difference. Since we do not observe the sparticles in nature, SUSY should be spontaneously broken not to spoil the cancellation of quadratic divergencies [31].

The MSSM respects the same $\mathrm{SU}(3)_{\mathrm{C}} \times \mathrm{SU}(2)_{\mathrm{L}} \times \mathrm{U}(1)_{\mathrm{Y}}$ gauge symmetries as does the SM [32] and assumes that the interaction between particles conserves R- 
Table 4.1: The MSSM chiral and gauge supermultiplets [30].

\begin{tabular}{|c|c|c|c|c|}
\hline Supermultiplets & Particle Content & $\mathrm{SU}(3)_{C}$ & $\mathrm{SU}(2)_{L}$ & $\mathrm{U}(1)_{Y}$ \\
\hline$\widehat{G}$ & $g, \tilde{g}$ & 8 & 1 & 0 \\
$\widehat{W}$ & $W, \widetilde{W}$ & 1 & 3 & 0 \\
$\widehat{B}$ & $B, \widetilde{B}$ & 1 & 1 & 0 \\
$\widehat{Q}$ & $(u, d)_{L},(\tilde{u}, \tilde{d})_{L}$ & 3 & 2 & $1 / 6$ \\
$\widehat{U}^{c}$ & $\bar{u}_{R}, \tilde{u}_{R}^{*}$ & $\overline{3}$ & 1 & $-2 / 3$ \\
$\widehat{D}^{c}$ & $\bar{d}_{R}, \tilde{d}_{R}^{*}$ & $\overline{3}$ & 1 & $1 / 3$ \\
$\widehat{L}$ & $(\nu, e)_{L},(\tilde{\nu}, \tilde{e})_{L}$ & 1 & 2 & $-1 / 2$ \\
$\widehat{E}^{c}$ & $\bar{e}_{R}, \tilde{e}_{R}^{*}$ & 1 & 1 & 1 \\
$\widehat{H}_{1}$ & $\left(H_{1}, \tilde{h}_{1}\right)$ & 1 & 2 & $-1 / 2$ \\
$\widehat{H}_{2}$ & $\left(H_{2}, \tilde{h}_{2}\right)$ & 1 & 2 & $1 / 2$ \\
\hline
\end{tabular}

parity. The $\mathrm{R}$ parity is a multiplicative quantum number and it is given as $\mathrm{R}=$ $(-1)^{3(B-L)+2 S}$, where $\mathrm{B}$ is the baryon number, $\mathrm{L}$ is the lepton number, and $\mathrm{S}$ is the spin number. The consequences of the $\mathrm{R}$ parity conservation are such that all sparticles are created in pairs, and the lightest supersymmetric particle (LSP) is stable and weakly interacts with particles. Therefore the generic experimental signature for R-parity conserving SUSY is large missing energy from a non-observed LSP and multijets from long cascade decays of sparticles.

The supersymmetry is one of the most studied Beyond the Standard Model (BSM) theories since it can:

- solve the "hierarchy problem". Since bosons and fermions contribute to one loop correction with opposite sign, the contributions to the mass term cancel in each order in perturbation theory and the correction does not increase quadratically.

- explain the source of the cold dark matter in the universe by providing a particle. 
The LSP is the leading candidate for dark matter.

- unify the gauge couplings. The existence of supersymmetric particles in the $\mathrm{TeV}$ mass range forces the coupling constants to unify at a scale of $10^{16} \mathrm{GeV}$. The symmetry breaking mechanism of the MSSM model introduces a large number of free parameters, and having many parameters complicates the phenomenology. Therefore many constrained models were introduced to the literature to describe broken symmetries with fewer parameters [8]. At the Grand Unified Theory (GUT) scale [33], the supersymmetric sector can be described by using only 5 parameters which are

- $\mathrm{m}_{0}$, the universal scalar mass

- $\mathrm{m}_{1 / 2}$, the universal gaugino mass

- $\mathrm{A}_{0}$, the common trilinear soft SUSY breaking parameter

- $\operatorname{sign}(\mu)$, the sign of Higgs mass parameter

- $\tan \beta$, the ratio of the vacuum expectation values of the two Higgs doublets.

The supersymmetric models which are defined by using these sets of parameters are called the constrained MSSM (CMSSM) [34]. For a given value of $\mathrm{A}_{0}, \operatorname{sign}(\mu)$, and $\tan \beta$, the masses of the SUSY particles can be found in terms of $\mathrm{m}_{0}$ and $\mathrm{m}_{1 / 2}$ for the entire low energy spectrum. The approximate results for slepton and squark masses can be formulated as [30]

$$
\begin{gathered}
M_{\tilde{l}}^{2} \sim m_{0}{ }^{2} \\
M_{\tilde{q}}^{2} \sim m_{0}^{2}+4 m_{1 / 2}^{2}
\end{gathered}
$$




\subsection{SUSY Search at the LHC with CMS}

The following decay modes summarize the production of SUSY particles (gluinos, squarks, neutralinos, charginos and sleptons) at hadron colliders.

Production of gluinos can occur with the following processes

$$
\begin{gathered}
g g \rightarrow \tilde{g} \tilde{g} \\
q \bar{q} \rightarrow \tilde{g} \tilde{g} \\
g q \rightarrow \tilde{g} \tilde{q} \\
q \bar{q} \rightarrow \tilde{\chi}_{i}^{0} \tilde{g}
\end{gathered}
$$

On the other hand production of squarks can come from

$$
\begin{gathered}
g g \rightarrow \tilde{q} \tilde{\bar{q}} \\
q \bar{q} \rightarrow \tilde{q} \tilde{\bar{q}} \\
q q \rightarrow \tilde{q} \tilde{q} \\
g q \rightarrow \tilde{\chi}_{i}^{0} \tilde{q}
\end{gathered}
$$

Production of neutralinos and charginos can come from

$$
\begin{gathered}
q \bar{q} \rightarrow W^{*} \rightarrow \tilde{\chi}_{j}^{ \pm} \tilde{\chi}_{i}^{0} \tilde{q} \\
q \bar{q} \rightarrow Z^{*} \rightarrow \tilde{\chi}_{j}^{ \pm} \tilde{\chi}_{j}^{\mp}
\end{gathered}
$$

and production of sleptons from

$$
q \bar{q} \rightarrow \tilde{l}_{L}^{ \pm} \tilde{l}_{L}^{\mp}
$$




$$
\begin{aligned}
q \bar{q} & \rightarrow \tilde{l}_{R}^{ \pm} \tilde{l}_{R}^{\mp} \\
q \bar{q} & \rightarrow \tilde{l}_{L}^{ \pm} \tilde{\nu}_{L} \\
q \bar{q} & \rightarrow \tilde{\nu} \tilde{b} \nu
\end{aligned}
$$

At the LHC, production of gluinos and squarks $(\tilde{q} \tilde{q}, \tilde{g} \tilde{g}, \tilde{q} \tilde{g})$ are the dominant processes and the Feynman diagrams for their productions are given in Figure 4.1.

The CMS Collaboration covers as many experimental signatures as possible for SUSY discovery. To do that, a set of test points [8] is defined by varying the five CMSSM parameters. There are a total of 14 test points where the first ten of them (from LM1 to LM10) are low mass points and the last four are high mass points. A summary of 14 mass points with their corresponding five CMSSM parameters is given in Table 4.2.

Table 4.2: The CMSSM mass points at CMS [8].

\begin{tabular}{|c|c|c|c|c|c|}
\hline Mass Points & $\mathrm{m}_{0}$ & $\mathrm{~m}_{1 / 2}$ & $\mathrm{~A}_{0}$ & $\operatorname{sign}(\mu)$ & $\tan \beta$ \\
\hline LM1 & 60 & 250 & 0 & + & 10 \\
LM2 & 185 & 350 & 0 & + & 35 \\
LM3 & 330 & 240 & 0 & + & 20 \\
LM4 & 210 & 285 & 0 & + & 10 \\
LM5 & 230 & 360 & 0 & + & 10 \\
LM6 & 85 & 400 & 0 & + & 10 \\
LM7 & 3000 & 230 & 0 & + & 10 \\
LM8 & 500 & 300 & -300 & + & 10 \\
LM9 & 1450 & 175 & 0 & + & 50 \\
LM10 & 3000 & 500 & 0 & + & 10 \\
HM1 & 180 & 850 & 0 & + & 10 \\
HM2 & 350 & 800 & 0 & + & 35 \\
HM3 & 700 & 800 & 0 & + & 10 \\
HM4 & 1350 & 600 & 0 & + & 10 \\
\hline
\end{tabular}


The followings are the dominant decay modes and the branching ratios for the 14 CMSSM test points [8].

- LM1:

- $\mathrm{m}(\tilde{g}) \geq \mathrm{m}(\tilde{q}), \tilde{g} \rightarrow \tilde{q} q$ is the dominant mode for producing sparticles.

$-\mathrm{B}\left(\tilde{\chi}_{2}^{0} \rightarrow \tilde{l}_{R} l\right)=11.2 \%, \mathrm{~B}\left(\tilde{\chi}_{2}^{0} \rightarrow \tilde{\tau}_{1} \tau\right)=46 \%, \mathrm{~B}\left(\tilde{\chi}_{1}^{ \pm} \rightarrow \tilde{\nu}_{l} l\right)=36 \%$

- LM2:

- $\mathrm{m}(\tilde{g}) \geq \mathrm{m}(\tilde{q}), \tilde{g} \rightarrow \tilde{q} q$ is the dominant $\left(\tilde{b}_{1} b\right.$ is $\left.25 \%\right)$

$-\mathrm{B}\left(\tilde{\chi}_{2}^{0} \rightarrow \tilde{\tau}_{1} \tau\right)=96 \%, \mathrm{~B}\left(\tilde{\chi}_{1}^{ \pm} \rightarrow \tilde{\tau} \nu\right)=95 \%$

- LM3:

- $\mathrm{m}(\tilde{g})<\mathrm{m}(\tilde{q})$, hence $\tilde{g} \rightarrow \tilde{q} q$ is forbidden except $\mathrm{B}\left(\tilde{g} \rightarrow \tilde{b}_{1,2} b\right)=85 \%$

$-\mathrm{B}\left(\tilde{\chi}_{2}^{0} \rightarrow l l \tilde{\chi}_{1}^{0}\right)=3.3 \%, \mathrm{~B}\left(\tilde{\chi}_{2}^{0} \rightarrow \tau \tau \tilde{\chi}_{1}^{0}\right)=2.2 \%, \mathrm{~B}\left(\tilde{\chi}_{1}^{ \pm} \rightarrow W^{ \pm} \tilde{\chi}_{1}^{0}\right)=100 \%$

- LM4:

$-\mathrm{m}(\tilde{g}) \geq \mathrm{m}(\tilde{q}), \tilde{g} \rightarrow \tilde{q} q$ is the dominant with $\mathrm{B}\left(\tilde{g} \rightarrow \tilde{b}_{1} b\right)=24 \%$

$-\mathrm{B}\left(\tilde{\chi}_{2}^{0} \rightarrow Z^{0} \tilde{\chi}_{1}^{0}\right)=97 \%, \mathrm{~B}\left(\tilde{\chi}_{1}^{ \pm} \rightarrow W^{ \pm} \tilde{\chi}_{1}^{0}\right)=100 \%$

- LM5:

$-\mathrm{m}(\tilde{g}) \geq \mathrm{m}(\tilde{q}), \tilde{g} \rightarrow \tilde{q} q$ is the dominant with $\mathrm{B}\left(\tilde{g} \rightarrow \tilde{b}_{1} b\right)=19.7 \%$ and

$$
\mathrm{B}\left(\tilde{g} \rightarrow \tilde{t}_{1} t\right)=23.4 \%
$$

$-\mathrm{B}\left(\tilde{\chi}_{2}^{0} \rightarrow h^{0} \tilde{\chi}_{1}^{0}\right)=85 \%, \mathrm{~B}\left(\tilde{\chi}_{2}^{0} \rightarrow Z^{0} \tilde{\chi}_{1}^{0}\right)=11.5 \%, \mathrm{~B}\left(\tilde{\chi}_{1}^{ \pm} \rightarrow W^{ \pm} \tilde{\chi}_{1}^{0}\right)=97 \%$

- LM6:

$-\mathrm{m}(\tilde{g}) \geq \mathrm{m}(\tilde{q}), \tilde{g} \rightarrow \tilde{q} q$ is the dominant 
$-\mathrm{B}\left(\tilde{\chi}_{2}^{0} \rightarrow \tilde{l}_{L} l\right)=10.8 \%, \mathrm{~B}\left(\tilde{\chi}_{2}^{0} \rightarrow \tilde{\tau}_{1} \tau\right)=14 \%, \mathrm{~B}\left(\tilde{\chi}_{1}^{ \pm} \rightarrow \tilde{\nu}_{l} l\right)=44 \%$

- LM7:

- $\mathrm{m}(\tilde{g})=678 \mathrm{GeV}$, hence $\tilde{g} \rightarrow 3$-body decay is dominant

$-\mathrm{B}\left(\tilde{\chi}_{2}^{0} \rightarrow l l \tilde{\chi}_{1}^{0}\right)=10 \%, \mathrm{~B}\left(\tilde{\chi}_{1}^{ \pm} \rightarrow \nu l \tilde{\chi}_{1}^{0}\right)=33 \%$

- $73 \%$ of the total cross-section comes from EW chargino-neutralino production

- LM8:

$-\mathrm{m}(\tilde{g})=745 \mathrm{GeV}, \mathrm{M}\left(\tilde{t}_{1}\right)=548 \mathrm{GeV}, \tilde{g} \rightarrow \tilde{t}_{1} t$ is dominant

$-\mathrm{B}\left(\tilde{g} \rightarrow \tilde{t}_{1} t\right)=81 \%, \mathrm{~B}\left(\tilde{g} \rightarrow \tilde{b}_{1} b\right)=14 \%, \mathrm{~B}\left(\tilde{q}_{L} \rightarrow q \tilde{\chi}_{2}^{0}\right)=26-27 \%$

$-\mathrm{B}\left(\tilde{\chi}_{2}^{0} \rightarrow Z^{0} \tilde{\chi}_{1}^{0}\right)=100 \%, \mathrm{~B}\left(\tilde{\chi}^{ \pm} \rightarrow W^{ \pm} \tilde{\chi}_{1}^{0}\right)=100 \%$

- LM9:

- $\mathrm{m}(\tilde{g})=507 \mathrm{GeV}$, hence $\tilde{g} \rightarrow 3$-body decay is dominant

$-\mathrm{B}\left(\tilde{\chi}_{2}^{0} \rightarrow l l \tilde{\chi}_{1}^{0}\right)=6.5 \%, \mathrm{~B}\left(\tilde{\chi}^{ \pm} \rightarrow \nu l \tilde{\chi}_{1}^{0}\right)=22 \%$

- LM10:

- $\mathrm{m}(\tilde{g})=1295 \mathrm{GeV}$, hence $\tilde{g} \rightarrow 3$-body decay is dominant

$-\mathrm{B}\left(\tilde{g} \rightarrow t \bar{t} \tilde{\chi}_{4}^{0}\right)=11 \%, \mathrm{~B}\left(\tilde{g} \rightarrow t b \tilde{\chi}_{2}^{ \pm}\right)=27 \%$

\section{- HM1:}

$-\mathrm{m}(\tilde{g}) \geq \mathrm{m}(\tilde{q}), \tilde{g} \rightarrow \tilde{q} q$ is the dominant

$-\mathrm{B}\left(\tilde{g} \rightarrow \tilde{t}_{1} t\right)=25 \%, \mathrm{~B}\left(\tilde{q}_{L} \rightarrow q \tilde{\chi}_{2}^{0}\right)=32 \%$

$-\mathrm{B}\left(\tilde{\chi}_{2}^{0} \rightarrow \tilde{l}_{L} l\right)=27 \%, \mathrm{~B}\left(\tilde{\chi}_{2}^{0} \rightarrow \tilde{\tau}_{1} \tau\right)=14 \%, \mathrm{~B}\left(\tilde{\chi}_{1}^{ \pm} \rightarrow \tilde{\nu}_{l} l\right)=37 \%$ 
- HM2:

$-\mathrm{m}(\tilde{g}) \geq \mathrm{m}(\tilde{q}), \tilde{g} \rightarrow \tilde{q} q$ is the dominant

$-\mathrm{B}\left(\tilde{g} \rightarrow \tilde{t}_{1} t\right)=25 \%, \mathrm{~B}\left(\tilde{q}_{L} \rightarrow q \tilde{\chi}_{2}^{0}\right)=32 \%$

$-\mathrm{B}\left(\tilde{\chi}_{2}^{0} \rightarrow \tilde{\tau}_{1} \tau\right)=78 \%, \mathrm{~B}\left(\tilde{\chi}_{1}^{ \pm} \rightarrow \tilde{\nu} \tau+\tilde{\tau}_{1} \nu\right)=13+76 \%$

\section{- HM3:}

$-\mathrm{m}(\tilde{g}) \geq \mathrm{m}(\tilde{q}), \tilde{g} \rightarrow \tilde{q} q$ is the dominant

$-\mathrm{B}\left(\tilde{g} \rightarrow \tilde{t}_{1} t\right)=52 \%, \mathrm{~B}\left(\tilde{q}_{L} \rightarrow q \tilde{\chi}_{2}^{0}\right)=32 \%$

$-\mathrm{B}\left(\tilde{\chi}_{2}^{0} \rightarrow h^{0} \tilde{\chi}_{1}^{0}\right)=94 \%, \mathrm{~B}\left(\tilde{\chi}_{1}^{ \pm} \rightarrow W^{ \pm} \tilde{\chi}_{1}^{0}\right)=100 \%$

\section{- HM4:}

$-\mathrm{m}(\tilde{g})<\mathrm{m}(\tilde{q})$, hence $\tilde{q} \rightarrow \tilde{g} q$ is important

$-\mathrm{B}\left(\tilde{q}_{L} \rightarrow \tilde{g} q\right)=43 \%, \mathrm{~B}\left(\tilde{q}_{R} \rightarrow \tilde{g} q\right)=77-93 \%, \mathrm{~B}\left(\tilde{g} \rightarrow \tilde{t}_{1} t\right)=82 \%$

$-\mathrm{B}\left(\tilde{t}_{1} \rightarrow t \tilde{\chi}_{2}^{0}\right)=3 \%, \mathrm{~B}\left(\tilde{t}_{1} \rightarrow t \tilde{\chi}_{3}^{0}\right)=22 \%, \mathrm{~B}\left(\tilde{t}_{1} \rightarrow t \tilde{\chi}_{4}^{0}\right)=16 \%$

$-\mathrm{B}\left(\tilde{\chi}_{2}^{0} \rightarrow h^{0} \tilde{\chi}_{1}^{0}\right)=94 \%, \mathrm{~B}\left(\tilde{\chi}_{4}^{0} \rightarrow h^{0} \tilde{\chi}_{2}^{0}\right)=30 \%, \mathrm{~B}\left(\tilde{\chi}_{1}^{ \pm} \rightarrow W^{ \pm} \tilde{\chi}_{1}^{0}\right)=100 \%$

A typical example with multijets and large missing transverse energy final state for $\tilde{g} \rightarrow \tilde{q} q$ decay is shown in Figure 4.2 .

To discover physics beyond the SM, the CMS experiment is looking for different generic signals, and multijet events (at least 3 jets) with a large missing transverse momentum $\left(h_{\mathrm{T}}\right)$ is one of them where $h_{\mathrm{T}}$ is defined as the magnitude of the negative vectorial sum of the transverse momenta of the jets. In this thesis, a search for multijet events with large $h_{\mathrm{T}}$ is presented for $36 \mathrm{pb}^{-1}$ of $\sqrt{\mathrm{s}}=7 \mathrm{TeV}$ proton-proton data collected with the CMS detector from 2010 March to 2010 November. Results 
are presented in the context of the CMSSM, and in the more general context by using simplified models [35-37].

\subsection{Event Reconstruction in CMS}

All the physics objects used in this analysis are reconstructed by the particle flow (PF) algorithm [38]. The PF algorithm was developed to identify almost all stable particles in the event with a good estimation of their energy, momentum, and type. The PF algorithm uses all the sub-detectors, charged particle tracks, calorimeter clusters and muon tracks, to optimize the particle reconstruction. Then it uses all those reconstructed particles to construct jets, to form missing transverse energy $\left(\mathrm{E}_{\mathrm{T}}\right)$, and to identify $\tau_{\mathrm{s}}$ and their decay products. The jets are reconstructed by using anti- $k_{T}$ algorithm with parameter $\mathrm{D}=0.5$ [39]. The anti- $k_{T}$ algorithm is a sequential clustering algorithm which produces circular cone-shaped jets. The jets used in this analysis are corrected for the non-linear calorimeter response with respect to their transverse momentum $\left(\mathrm{p}_{\mathrm{T}}\right)$ and $\eta$ by the correction factors derived from simulations [40]. In addition to that, jets in data are corrected with the residual correction, which is derived from the data in order to account for jet response difference in data and in simulation samples.

\subsection{Sample Selection}

The aims of the event selection used in this analysis are

1. to be inclusive so that many new physics models with hadronic final states with missing momentum can be observed. 
2. to be efficient for new physics models with small mass particles.

3. to define a search region where SM backgrounds can be estimated by using data-driven methods.

The data used in this analysis are collected with different trigger paths based

on the quantity $\mathrm{H}_{\mathrm{T}}^{\text {trigger }}$, defined as the scalar sum of the transverse momenta of the reconstructed calorimeter jets with $\mathrm{p}_{\mathrm{T}}>20 \mathrm{GeV}$. Because of the instantaneous luminosity increase at $\mathrm{LHC}$, the threshold on the $\mathrm{H}_{\mathrm{T}}^{\text {trigger }}$ was raised from 100, to 140 , and to $150 \mathrm{GeV}$ during the 2010 data-taking period.

Many non-physical effects can mimic the actual final state signatures. These effects can come from real particles, which originate from the beam backgrounds, cosmic rays, muons, and also from the detector noise, which can cause fake energy deposits in the calorimeter. To clean the non-physical effects from real collisions, the possible sources of fake $h_{\mathrm{T}}$, various sources of detector noise, and beam related background events are removed from the data by applying several cleanup and quality requirements [41-43].

The event selection in this analysis starts with loose requirements. Later on in this analysis tighter requirements are applied to define the search selections. The list of event selections after the trigger selection is as follow:

- At least 3 jets with $p_{T}>50 \mathrm{GeV}$ and $|\eta|<2.5$. The selection on pseudorapidity is restricted to 2.5 to have the full jet energy deposit contained in the barrel and endcap regions of the calorimeters.

- $\mathrm{H}_{\mathrm{T}}>300 \mathrm{GeV}$, where $\mathrm{H}_{\mathrm{T}}$ defined as the scalar sum of the transverse momenta 
of the jets with $p_{T}>50 \mathrm{GeV}$ and $|\eta|<2.5$. This requirement is chosen to be above the initial $\mathrm{H}_{\mathrm{T}}$ trigger.

- $H_{\mathrm{T}}>150 \mathrm{GeV}$, where $\mathrm{H}_{\mathrm{T}}$ is defined as the magnitude of the negative vectorial sum of the transverse momenta of the jets where jets are required to satisfy $p_{T}>30 \mathrm{GeV}$ and $|\eta|<5$. This requirement extinguishes most of the QCD multijet events.

- $\mid \Delta \phi\left(\right.$ jet $\left._{1,2}, H_{\mathrm{T}}\right) \mid>0.5 \mathrm{rad}$ and $\mid \Delta \phi\left(\right.$ jet $\left._{3}, H_{\mathrm{T}}\right) \mid>0.3 \mathrm{rad}$. These requirements are introduced to remove most of the QCD multijet events in which a single mismeasured jet causes a high $\not_{\mathrm{T}}$. The $\mid \Delta \phi\left(\right.$ jet $\left._{1,2,3}, \mathbb{H}_{\mathrm{T}}\right) \mid$ distributions for the QCD and LM1 samples are shown in Figure 4.3.

- Vetoes on isolated muons and electrons. This requirement is applied to reject the leptonic final states of $\mathrm{W}+$ jets, $\mathrm{Z}+$ jets, and $\mathrm{t} \overline{\mathrm{t}}$ events. Muons and electrons are required to have $p_{T}>10 \mathrm{GeV}$ and a good quality track matched to the primary vertex. They are required to satisfy also the criteria $\left(\sum^{\Delta R<0.4} p_{T}^{\text {ch }}+\right.$ $\left.\sum^{\Delta R<0.4} p_{T}^{\mathrm{nh}}+\sum^{\Delta R<0.4} p_{T}^{\gamma}\right) / p_{T}^{\text {lepton }}<0.2$, where $p_{T}^{\mathrm{ch}}, p_{T}^{\mathrm{nh}}, p_{T}^{\gamma}$ and $p_{T}^{\text {lepton }}$ are the momenta of charged hadrons, neutral hadrons and photons. The pseudorapidity condition for muons and electrons, respectively, is $|\eta|<2.4$ and 2.5, excluding the transition region $1.44<|\eta|<1.57$.

Two search regions are chosen based on the observables central to this inclusive multijet and missing momentum search [44]:

1. High $\bigsqcup_{\mathrm{T}}$ search region: $\bigsqcup_{\mathrm{T}}>250 \mathrm{GeV}$, motivated by the search for generic dark matter candidates coupled with high background rejection. 
Table 4.3: List of the MC

datasets and corresponding cross

sections.

\begin{tabular}{|l|cc|}
\hline Dataset & $\sigma(\mathrm{pb})$ & \\
\hline $\mathrm{t} \overline{\mathrm{t}}$ & 165 & $(\mathrm{NNLO})$ \\
$\mathrm{W}(l \nu)+$ jets & 31300 & $(\mathrm{NNLO})$ \\
$\mathrm{Z}(\nu \bar{\nu})+$ jets & 5768 & $(\mathrm{NNLO})$ \\
$\mathrm{LM} 1$ & 6.5 & $(\mathrm{NLO})$ \\
\hline
\end{tabular}

2. High $\mathrm{H}_{\mathrm{T}}$ search region: $\mathrm{H}_{\mathrm{T}}>500 \mathrm{GeV}$, sensitive to higher object multiplicities like SUSY cascade decays.

\subsection{Monte Carlo Production}

To compare our results with SM backgrounds we used several Monte Carlo (MC) simulated signal and background samples that are produced with detailed Geant4 [45] CMS detector simulation. The QCD multijet, $\mathrm{t} \overline{\mathrm{t}}, \mathrm{W}, \mathrm{Z}, \gamma+$ jets, dibosons and single top samples are generated with PYTHIA [46] and MADGRAPH [47] generators by using the CTEQ6.1L [48] parton distribution functions. The LM1 CMSSM point [8], with mSUGRA parameters $m_{0}=60 \mathrm{GeV}, m_{1 / 2}=250 \mathrm{GeV}, A_{0}=0, \tan \beta=10$, and $\operatorname{sign}(\mu)>0$, is used as our benchmark point.

The predicted cross sections for MC samples are normalized to next-to-leading (NLO) or next-to-next-to-leading-order (NNLO) cross sections when available [49-51] and the event yield is normalized to the total integrated luminosity of $36 \mathrm{pb}^{-1}$. The samples with NLO and NNLO corrected cross sections are summarized in Table 4.3. 


\subsection{The SM Backgrounds}

The largest SM backgrounds for the multijet and large $H_{\mathrm{T}}$ analysis come from $\mathrm{QCD}, \mathrm{Z}(\rightarrow \nu \nu)+$ jets, $\mathrm{W}+$ jets and $t \bar{t}$, and multijet events with large missing momentum from leptonic decays of quarks. There are also contributions due to jet mismeasurements, and noise or dead components from the detector. Since QCD background is the biggest for this analysis, the next chapter (Chapter 5) is devoted to the QCD background estimation method and jet resolution measurements.

The event yields for the data and MC samples after the trigger, cleaning and event selections are tabulated in Table 4.4. The $\mathrm{H}_{\mathrm{T}}$ and $\mathrm{H}_{\mathrm{T}}$ distributions after the baseline selection are shown in Figures 4.4 and 4.5. The simulations are only used for verification, since all of the SM backgrounds in this analysis are estimated by using data-driven methods.

\subsubsection{Z $(\nu \bar{\nu})+$ jets Background Estimation}

The $\mathrm{Z}+$ jets events followed by neutrino decay of the $\mathrm{Z}$ boson are an irreducible background for multijet and large missing transverse momentum events. We estimate the number of $Z+$ jets events by using $\gamma+$ jets sample since the electroweak behavior of the $\mathrm{Z}$ boson and the photon are similar for high $\mathrm{p}_{\mathrm{T}}$ region. The differences that should be taken into account come from the electroweak couplings and the asymptotically vanishing residual mass effects [52]. The ratio of the $\mathrm{Z}$ boson and $\gamma+$ jets production cross section provides a good handle for estimating the missing momentum spectrum for $\mathrm{Z}+$ jets events for the higher $\mathrm{p}_{\mathrm{T}}$ region. 
Table 4.4: Event yields in data and MC samples for the largest SM backgrounds.

\begin{tabular}{|l|c|c|c|}
\hline & Baseline Sel. & High- $\boldsymbol{h}_{\mathrm{T}}$ Sel. & High- $\mathrm{H}_{\mathrm{T}}$ Sel. \\
\hline Data & 111 & 15 & 40 \\
SM Prediction & 81.2 & 12.9 & 26.4 \\
\hline $\mathrm{QCD}$ & 16.9 & 0.5 & 9.3 \\
$\mathrm{Z} \rightarrow \nu \bar{\nu}$ & 21.1 & 6.3 & 5.7 \\
$\mathrm{~W}$ & 19.7 & 3.5 & 5.6 \\
$t \bar{t}$ & 23.1 & 2.7 & 6 \\
otherEWK & 1.6 & 0 & 0 \\
\hline LM1 (benchmark) & 44.4 & 30.9 & 33.2 \\
\hline
\end{tabular}

All the MC samples are generated with the MADGRAPH, except the QCD multijets and signal sample which are generated by PYTHIA [44].

The $\gamma+$ jets control sample we used in the $\mathrm{Z}(\nu \bar{\nu})+$ jets background estimation is collected using single photon triggers. To discriminate photons from electrons, we veto events with a track seed in the pixel detector. Photons coming from QCD events are suppressed by requiring isolated photons. Since only direct photons are related to the $\mathrm{Z}$ production, the $5 \%$ contribution from fragmentation photons [53], and from isolated neutral pions and $\eta$ mesons decaying to pairs of secondary photons (the purity of the prompt photon is found to be $\sim 94 \%$ ) are treated as background and subtracted from the $\gamma+$ jets control sample.

We obtain the $\mathrm{Z} / \gamma$ cross section correction factor using simulated $\gamma+$ jets and $\mathrm{Z}(\rightarrow \nu \bar{\nu})$ MADGRAPH samples. The following corrections are applied to the background subtracted $\gamma+$ jets control sample before the number of events passing the 
search selections are predicted for $\mathrm{Z}(\nu \bar{\nu})+$ jets events:

- cross section ratio between the $\mathrm{Z}(\nu \bar{\nu})+$ jets and $\gamma+$ jets processes

- photon selection and isolation cuts applied to the MC sample

- photon reconstruction inefficiency

- photon identification and isolation efficiencies

The total $\mathrm{Z} / \gamma$ correction factors with systematic uncertainties are $0.37 \pm 14 \%$ for the baseline, $0.45 \pm 18 \%$ for High- $\mathrm{H}_{\mathrm{T}}$, and $0.38 \pm 17 \%$ for High- $\mathrm{H}_{\mathrm{T}}$ selection [44]. The results of the $\mathrm{Z}(\nu \bar{\nu})+$ jets background estimation for different search regions are summarized in Table 4.5. We determine predictions by multiplying the numbers of $\gamma+$ jets events with the $\mathrm{Z} / \gamma$ correction factors. The predictions are in good agreement with the estimations derived from MC simulations.

Table 4.5: The number of $\gamma+$ jets events in data and estimated number of $\mathrm{Z}(\nu \bar{\nu})+$ jets background (with systematic and statistical errors).

\begin{tabular}{|l|c|c|c|}
\hline & Baseline Sel. & High- H $_{\mathrm{T}}$ Sel. & High- $\mathrm{H}_{\mathrm{T}}$ Sel. \\
\hline$\gamma+$ jets data sample & 72 & 16 & 22 \\
$\mathrm{Z} \rightarrow \nu \bar{\nu}$ (data) & $26.3 \pm 3.2 \pm 3.6$ & $7.1 \pm 1.8 \pm 1.3$ & $8.4 \pm 1.8 \pm 1.4$ \\
$\mathrm{Z} \rightarrow \nu \bar{\nu}(\mathrm{MC})$ & $21.1 \pm 1.4$ & $6.3 \pm 0.8$ & $5.7 \pm 0.7$ \\
\hline
\end{tabular}

The direct prediction from MC sample with statistical errors is also given. The data driven estimation is calculated by multiplying the number of $\gamma+$ jets events with $\mathrm{Z} / \gamma$ correction [44]. 


\subsection{2 $\mathrm{W}+$ jets and $t \bar{t}$ Background Estimation}

To eliminate SM events which come from the leptonic final state of $\mathrm{W}+$ jets, $\mathrm{Z}+$ jets, and $t \bar{t}$ events, a veto is applied on the isolated muons and electrons with $\mathrm{p}_{\mathrm{T}}$ $>10 \mathrm{GeV}$. However this requirement does not reject the events when:

- a lepton coming from a W or a top quark decay is outside the geometric or kinematic acceptance

- a lepton is not reconstructed

- a lepton is not isolated

- a tau lepton decays hadronically

The first three items are denoted as lost leptons, whereas the last item is denoted as a hadronic $\tau\left(\tau_{h}\right)$. We use two separate data-driven methods to estimate backgrounds from $\mathrm{W}+$ jets and $t \bar{t}$ processes. Both methods use $\mu+$ jet events as the control sample.

The first method estimates the number of events that fail the isolated lepton requirement, and the second method predicts the background which comes from hadronic $\tau$ decay by substituting the muon in the $\mu+$ jet sample with a $\tau$ jet.

The total number of events which come from the lost lepton and hadronic $\tau$ background predictions is considered as the sum of the $\mathrm{W}+$ jets and $t \bar{t}$ backgrounds. The procedures are summarized below.

\subsubsection{The $\mathbf{W} / t \bar{t} \rightarrow e, \mu+\mathbf{X}$ background estimation}

The estimate of $\mathrm{W}+$ jets and $t \bar{t}$ events (where $\mathrm{W}$ decays leptonically and the leptons are not vetoed by the isolated lepton requirement) is measured by using a 
muon control sample. The control sample consists of events with exactly one muon that passes all the isolation and quality cuts given in Section 4.4. We applied three corrections to the control sample in order to estimate the number of events in the signal region. The control sample is first corrected for non-isolated but identified electrons and muons, the second for non-identified electrons and muons. The last correction is to correct the control sample by $R_{\text {accept }}$, estimated by simulations, which is the ratio of the events without acceptance leptons to those within the acceptance. The corrections are obtained from data using Tag \& Probe method.

A total $18 \%$ systematical uncertainty is assigned to the background estimation. The sources of this uncertainty come from isolation and identification efficiencies, the kinematic difference between the signal and the control sample, the SM background contamination in the control sample, and the use of simulations for acceptance calculation [44].

The predictions are obtained from the muon samples, selected using the same $\mathrm{H}_{\mathrm{T}}$ triggers we use for the signal searches and compared to the predictions from the simulated $\mathrm{W}+$ jets and $t \bar{t}$ events by using the same method, and to the direct prediction from MC PYHTIA sample. The results with statistical and systematical uncertainties are summarized in Table 4.6. This background estimation method reproduces the MC expectations (MC PYHTIA) when it is applied to the simulation (Estimate from MC); however, the numbers from the data-driven estimation (Estimate from data) are $50 \%$ higher than the MC expectations. This difference is attributed to the MC generator parameter tunes in the MC samples used in this background estimation 
method [44].

Table 4.6: The estimated number of events for the lost lepton background when the number is extracted from the data (Estimate from data) or MC (Estimate from MC) with the statistical and systematical uncertainties [44].

\begin{tabular}{|l|l|l|l|}
\hline & Baseline Sel. & High- $\bigsqcup_{\mathrm{T}}$ Sel. & High- $\mathrm{H}_{\mathrm{T}}$ Sel. \\
\hline Estimate from data & $33.0 \pm 5.5_{-5.7}^{+6.0}$ & $4.8 \pm 1.8_{-0.6}^{+0.8}$ & $10.9 \pm 3.0_{-1.7}^{+1.7}$ \\
Estimate from MC & $22.9 \pm 1.3_{-2.6}^{+2.7}$ & $3.2 \pm 0.4_{-0.5}^{+0.5}$ & $7.2 \pm 0.7_{-1.1}^{+1.1}$ \\
MC PYHTIA & $23.6 \pm 1.0$ & $3.6 \pm 0.3$ & $7.8 \pm 0.5$ \\
\hline
\end{tabular}

\subsubsection{The $\mathrm{W} / t \bar{t} \rightarrow \tau_{h}+\mathrm{X}$ background estimation}

We use a muon+jet control sample to estimate the hadronic $\tau$ background. The control sample is chosen by single muon triggers. Events with exactly one muon with $\mathrm{p}_{\mathrm{T}}>20 \mathrm{GeV}$ and $|\eta|<2.1$ are used in the background estimation method. Muons are also required to pass the isolation and quality cuts described in Section 4.4.

The event kinematics of hadronic $\tau$ events are similar to those in the muon control sample except for the energy of the $\tau$ jets. This energy difference is corrected by replacing each muon in the control sample with a $\tau$ jet. The momentum of $\tau$ jets are obtained by scaling the muon momentum with a correction factor that is derived using a simulated energy response that models the fraction of visible momentum as a function of true lepton momentum $[54,55]$. 
The following criteria are applied to the control sample to estimate background:

- kinematic and geometrical acceptance for the muons; $0.84 \pm 0.05$ for the High$H_{\mathrm{T}}$ and baseline selections, $0.89 \pm 0.05$ for the High- $\mathrm{H}_{\mathrm{T}}$ selection,

- correction for the muon triggers, reconstruction and isolation efficiencies,

- correction for the relative branching ratio of $\mathrm{W}$ decays to muons or hadronic $\tau$ jets; a factor of 0.65 is applied for the simulated events whereas the relative fraction of 0.69 is applied when the results are extracted from the data [22].

The method is tested on both data and simulated $\mathrm{W} / t \bar{t}$ events. Within the uncertainties the data and simulation predict similar numbers of events. The systematic uncertainties come from the possible difference in data and MC for the $\tau$ energy response $(2 \%)$, the SM background for the muon control sample (5\%), the muon acceptance $(+6 \%,-5 \%)$, and muon efficiency measurements in the data (1\%) [44]. The predicted numbers of events for the hadronic $\tau$ background with the systematic uncertainties are summarized in Table 4.7 .

Table 4.7: Estimated number of events for the hadronic $\tau$ background from data and MC simulations with their statistical and systematic uncertainties [44].

\begin{tabular}{|c|c|c|c|}
\hline & Baseline Sel. & High- $_{\mathrm{T}}$ Sel. & High- $_{\mathrm{T}}$ Sel. \\
\hline $\mathrm{W} / t \bar{t} \rightarrow \tau_{h}$ (data) & $22.3 \pm 4.0 \pm 2.2$ & $6.7 \pm 2.1 \pm 0.5$ & $8.5 \pm 2.5 \pm 0.7$ \\
$\mathrm{~W} / t \bar{t} \rightarrow \tau_{h}$ (MC) & $19.9 \pm 0.9$ & $3.0 \pm 0.4$ & $5.5 \pm 0.5$ \\
\hline
\end{tabular}


Since QCD is one of the main backgrounds and most of my efforts are directed toward to the jet resolution measurements for the QCD background estimation method, the next chapter is devoted to the QCD background estimation. 


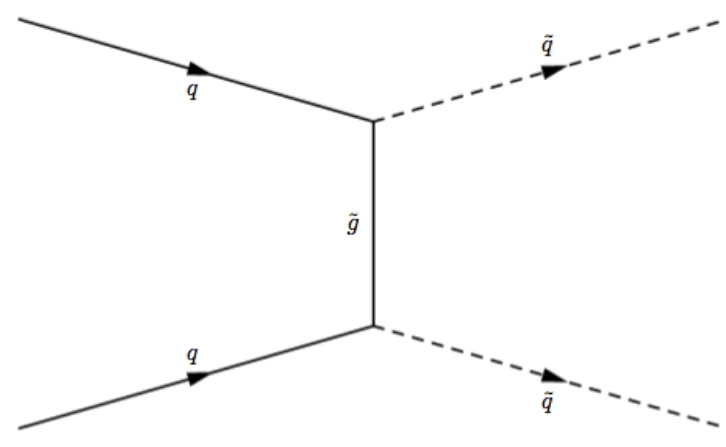

(a) $q q \rightarrow \tilde{q} \tilde{q}$

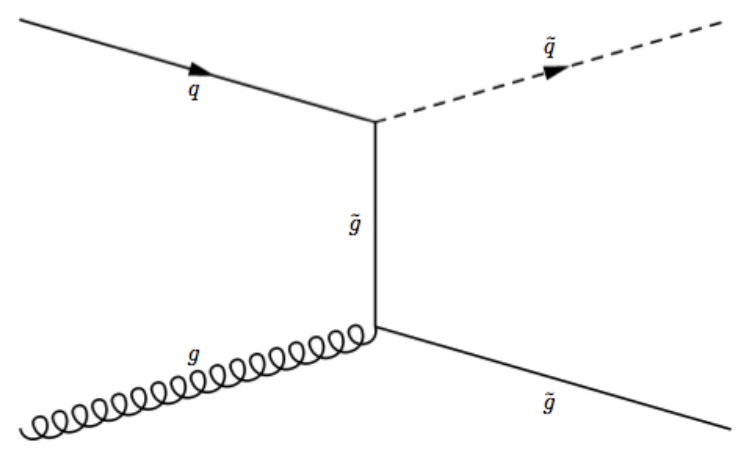

(b) $q g \rightarrow \tilde{q} \tilde{g}$

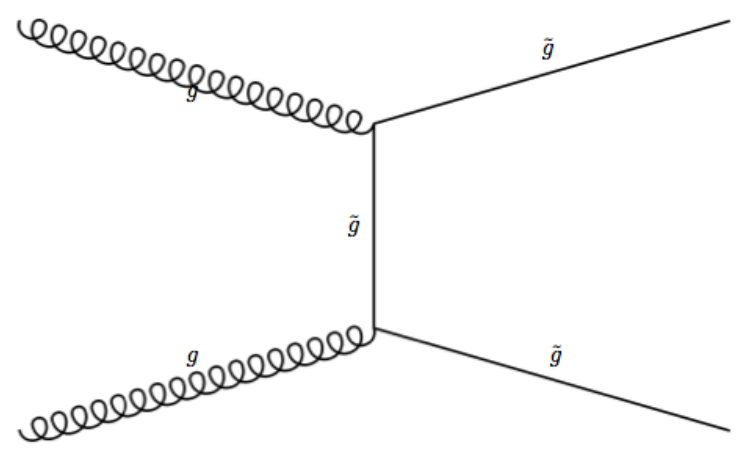

(c) $g g \rightarrow \tilde{g} \tilde{g}$

Figure 4.1: Feynman diagrams for $\tilde{q} \tilde{q}(\mathrm{a}), \tilde{q} \tilde{g}(\mathrm{~b})$, and $\tilde{g} \tilde{g}$ (c) productions. 


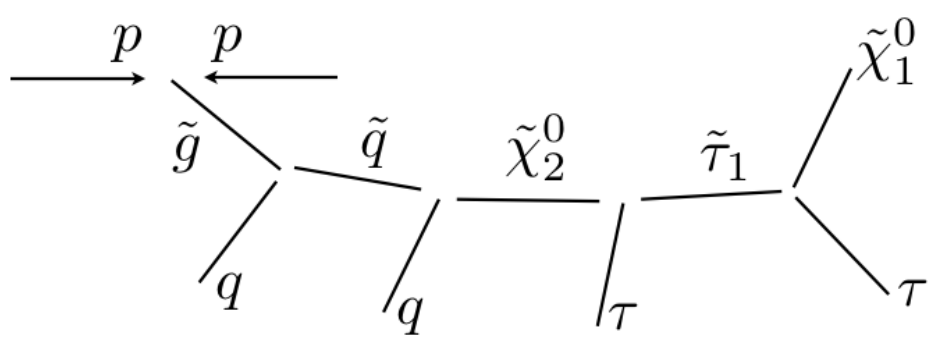

Figure 4.2: A typical example for $\tilde{g} \rightarrow \tilde{q} q$ decay with multijets (quarks and taus) and large missing transverse energy $\left(\tilde{\chi}_{1}^{0}\right)$ final state for CMS LM1 benchmark point.
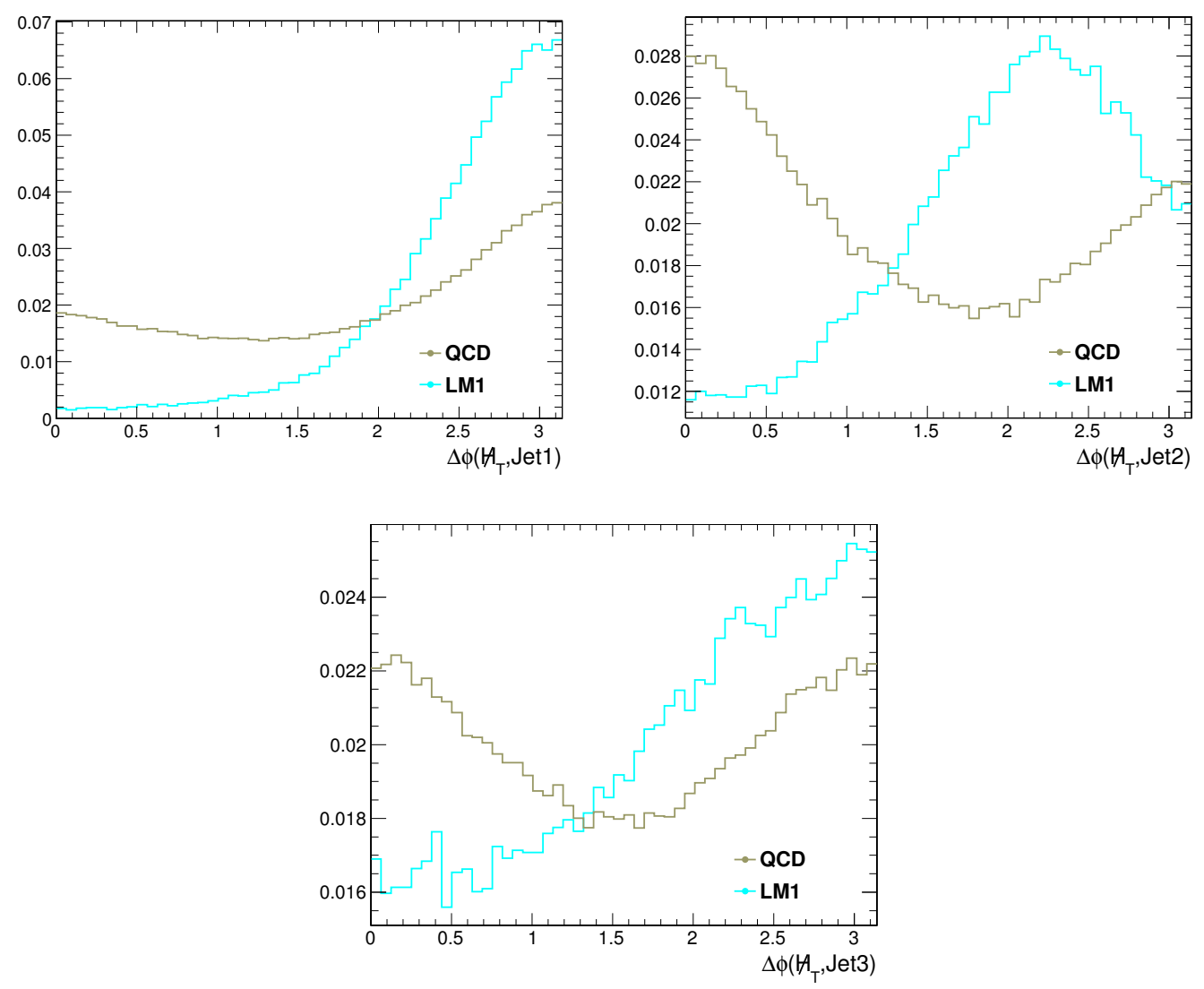

Figure 4.3: $\Delta \phi$ distributions between the first three leading jets and the $\not_{\mathrm{T}}$ for the QCD and LM1 samples. All histograms are normalized to unit area. 

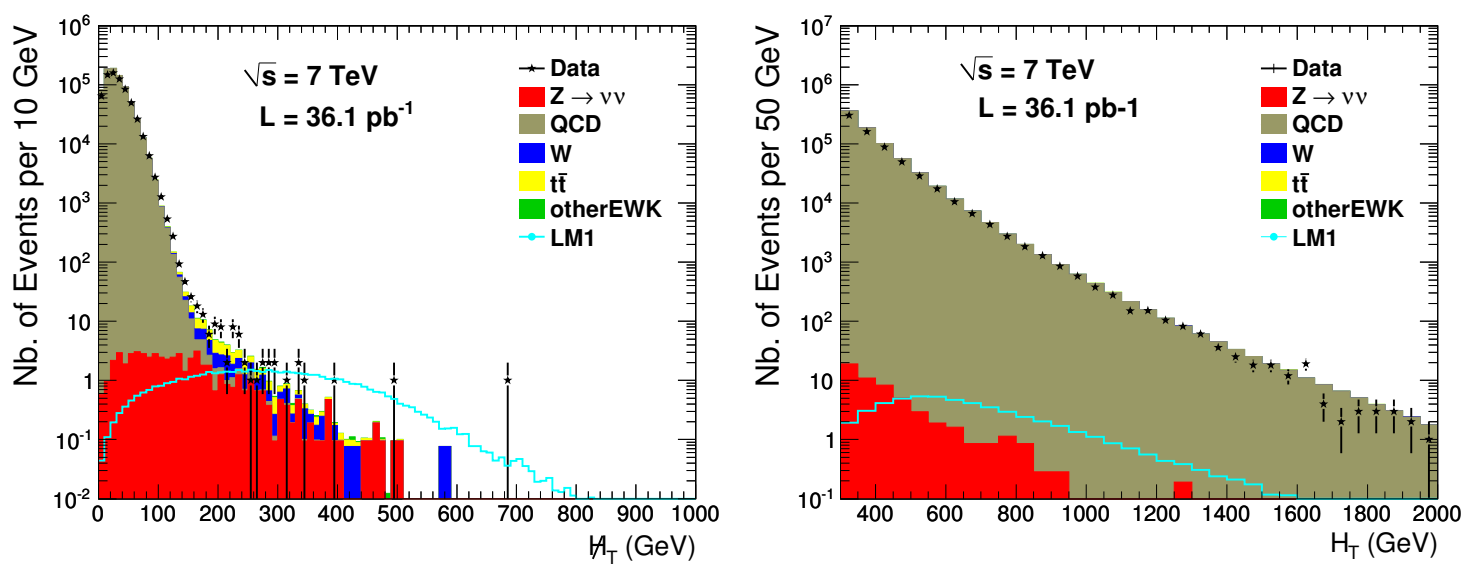

Figure 4.4: $H_{\mathrm{T}}$ (left) and $\mathrm{H}_{\mathrm{T}}$ (right) distributions for the data and $\mathrm{MC}$ samples after baseline selections except $H_{\mathrm{T}}>150 \mathrm{GeV}$ requirement.
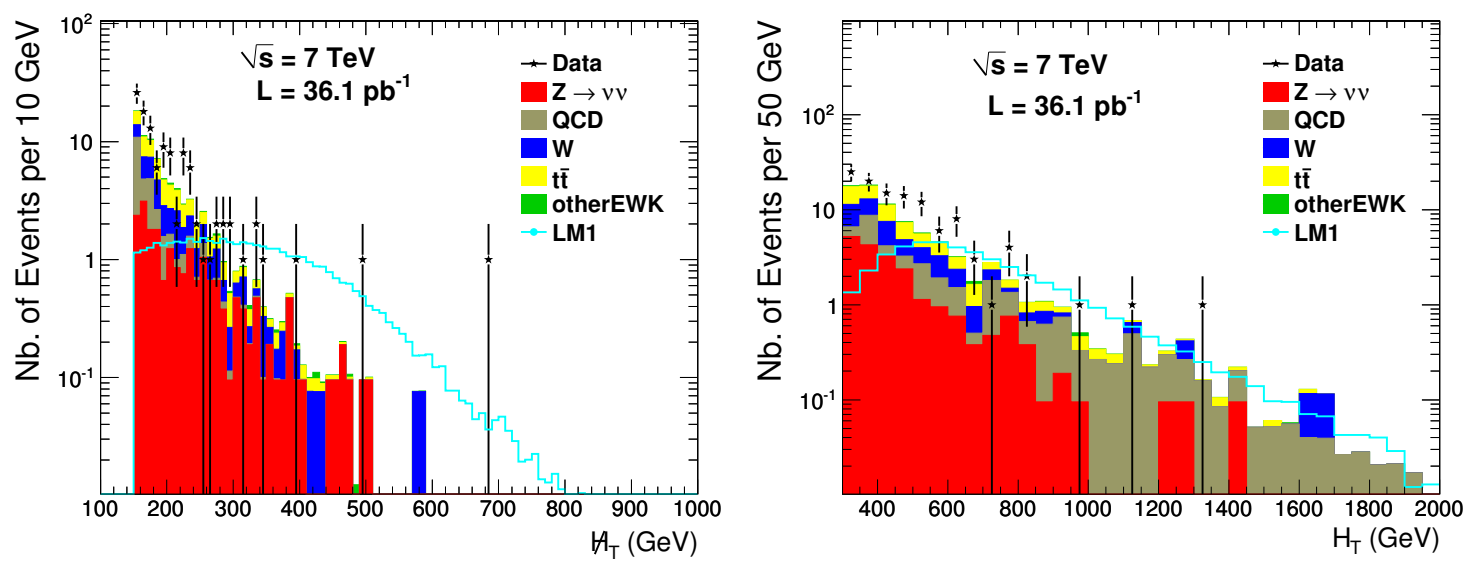

Figure 4.5: $\mathrm{H}_{\mathrm{T}}$ (left) and $\mathrm{H}_{\mathrm{T}}$ (right) distributions for the data and $\mathrm{MC}$ samples after baseline selections including the $H_{\mathrm{T}}>150 \mathrm{GeV}$ requirement. 


\section{CHAPTER 5 \\ QCD BACKGROUND ESTIMATION}

\subsection{Introduction}

Due to their large production cross-section, QCD multijet events constitute a large background to the jets $+H_{\mathrm{T}}$ final state. Large fluctuations in the jet energy response result in large $H_{T}$ in well-balanced QCD events. In addition to this, the semi-leptonic decays of heavy flavor quarks and dead/malfunctioning channels can also contribute to this imbalance in QCD events. These effects manifest themselves as deviations from the Gaussian nature of jet resolutions. Therefore it is very important to measure the complete jet resolution functions (Gaussian core + non-Gaussian tails) to be able to estimate the QCD background to jets $+H_{\mathrm{T}}$ final states from the collision data.

In this analysis, we use the Rebalance and Smear (R\&S) method [44] to estimate the QCD background directly from the data. In addition to predicting event counts, this method also predicts the full kinematics for multijet events such as the $H_{\mathrm{T}}$ spectrum while being unaffected by the presence of signal events in the sample that is used to predict the background.

The R\&S method starts with rebalancing the reconstructed multijet events with at least two jets with $\mathrm{p}_{\mathrm{T}}>10 \mathrm{GeV}$ to produce "seed events" which are a good estimation for the true well-balanced (no missing momentum) QCD events. The multijet sample is collected by requiring the triggers listed in Section 4.4. Then we 
smear the seed events to estimate the QCD background for multijet $+H_{\mathrm{T}}$ events. We use jet energy resolutions both in rebalancing (Gaussian resolutions) and smearing (full resolutions) procedures.

The rebalancing procedure, the four-momenta of jets are adjusted by using jet resolution functions measured from data and the events are brought into transverse momentum balance. The rebalancing procedure converts even high missing momentum events from neutrinos and other undetected particles to the well-balanced QCD events [44]. Other SM backgrounds, $\mathrm{Z}(\rightarrow \nu \nu)+$ jets, $\mathrm{W}+$ jets and $t \bar{t}$, have negligible effect on the rebalancing step since their production rates are very small compared to the QCD multijet production rates.

As a next step, the momentum of each jet in the seed event sample is smeared using a random number from measured jet resolution distributions. The smeared events are used to predict the kinematic properties such as $H_{\mathrm{T}}$ and $H_{\mathrm{T}}$ distributions after the search requirements are applied. The R\&S method is applied on simulated events, and predicted distributions are compared with the original reconstructed MC distributions as shown in Figure 5.1. We can predict the kinematic distributions $\left(\not_{\mathrm{T}}\right.$ and $\mathrm{H}_{\mathrm{T}}$ ) within $10 \%$ of the original $\mathrm{MC}$ distributions for the baseline and high $\mathrm{H}_{\mathrm{T}}$ selections. The disagreement between the predictions and actual MC distribution goes up to $40 \%$ for the high $\not_{\mathrm{T}}$ region; however, this is the region where the QCD multijet contribution is negligible compared to the other SM backgrounds. The number of predicted events in the simulation and smeared seed events after the search selections are given in Table 5.1. 
Jet energy resolutions provide crucial inputs to the R\&S QCD background estimation method, and in the following section the measurement of jet energy resolutions from the $\gamma+$ jet and dijet data is given in detail.
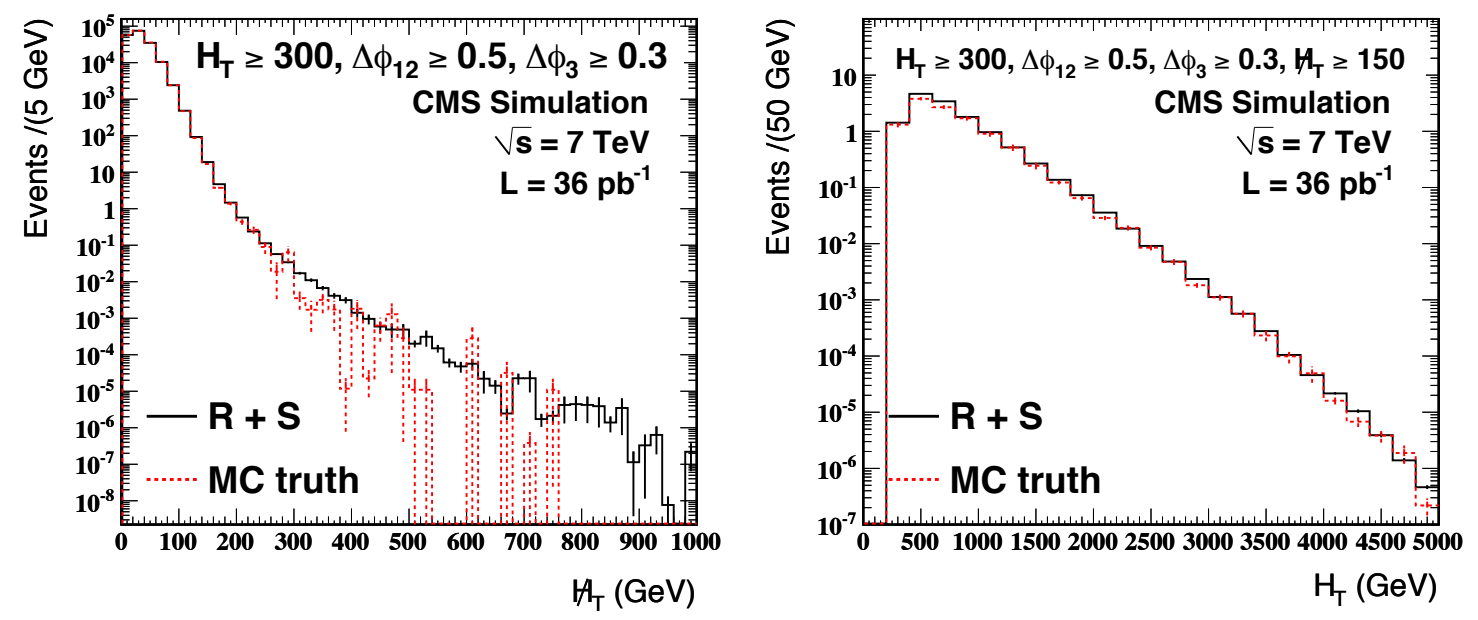

Figure 5.1: The $H_{\mathrm{T}}$ (left) and $\mathrm{H}_{\mathrm{T}}$ (right) distributions from the rebalance and smear method applied to simulation events and compared to actual MC (MC truth) distributions for events passing multijet, $\mathrm{H}_{\mathrm{T}} \geq 300 \mathrm{GeV}$, and $\Delta \phi\left(\right.$ jet $\left._{1-3}, H_{\mathrm{T}}\right)$ selections. Additionally the $\not_{\mathrm{T}}>150 \mathrm{GeV}$ requirement is applied for the right plot [44].

\subsection{Jet Energy Resolution Measurements}

There are several methods which use collider data to derive the full jet energy resolution functions, including the non-Gaussian tails. Although these methods rely on momentum conservation in the transverse plane, they differ in techniques such as a 
Table 5.1: Number of events predicted after search selection estimated from PYTHIA multijet sample.

\begin{tabular}{|l|c|c|c|}
\hline & Baseline Sel. & High- $\boldsymbol{H}_{\mathrm{T}}$ Sel. & High- $\mathrm{H}_{\mathrm{T}}$ Sel. \\
\hline $\mathrm{N}(\mathrm{PYTHIA})$ & $11.4 \pm 0.4$ & $0.13 \pm 0.04$ & $8.46 \pm 0.32$ \\
\hline $\mathrm{N}(\mathrm{R} \& \mathrm{~S})$ & $13.2 \pm 0.1$ & $0.177 \pm 0.004$ & $9.57 \pm 0.04$ \\
\hline $\mathrm{N}(\mathrm{R} \& \mathrm{~S}) / \mathrm{N}(\mathrm{PYTHI})$ & $1.15 \pm 0.04$ & $1.4 \pm 0.4$ & $1.13 \pm 0.05$ \\
\hline
\end{tabular}

The number of events predicted after search selection based on applying R\&S method to PYTHIA multijet sample. Only statistical uncertainties are given [44].

maximum likelihood approach in a global fit to dijet events [56], $\mathrm{p}_{\mathrm{T}}$ balance in $\gamma+$ jets events [57] or missing $\mathrm{p}_{\mathrm{T}}$ fraction in the direction of the photon (MPF) [58]. In this study, I focus on measuring jet energy resolutions from $\gamma+$ jets and dijet events using the $\mathrm{p}_{\mathrm{T}}$ balance technique and dijet asymmetry method.

The jet energy resolutions are measured both in data and simulated samples $(\mathrm{MC})$. The $\gamma+$ jet samples are used to measure the resolutions for the lower $\mathrm{p}_{\mathrm{T}}$ region, whereas the dijet samples are used for the higher $\mathrm{p}_{\mathrm{T}}$ region. The disagreement between data and MC is quantified as the ratio of measured resolutions (Data/MC) and used to modify $\mathrm{MC}$ truth resolutions according to the difference between the data and MC, and then adjusted resolutions are used in the R\&S method. The MC truth resolution is measured as $\mathrm{p}_{\mathrm{T}}^{\mathrm{Jet}} / \mathrm{p}_{\mathrm{T}}^{\text {ParticleJet }}$, where particle jet is the generator-level jet, and its transverse momentum is calculated by perfectly measuring the momentum of all the particles in the jet. The resolution functions are also used to estimate sys- 
tematic uncertainties due to the jet resolution measurements in the QCD background estimation, and for signal efficiency calculations for discovery or exclusion limits.

I performed another study to estimate the desired lower limit on the jet $\mathrm{p}_{\mathrm{T}}$ that can be used in jet energy resolution measurements. I found that we need to go as low as $20 \mathrm{GeV}$ on jet $\mathrm{p}_{\mathrm{T}}$ to minimize our error in estimating the $\mathrm{QCD} \mathrm{H}_{\mathrm{T}}$ distribution. The details are described in Appendix A.

\subsection{1 $\gamma+$ Jet Measurements}

Since the photons are reconstructed with a excellent energy resolution $(\sim$ 1\%) [59], the width of a $\mathrm{p}_{\mathrm{T}}^{\mathrm{Jet}} / \mathrm{p}_{\mathrm{T}}^{\gamma}$ distribution in events with a $\gamma+$ jet final state, which is denoted by $\sigma\left(\mathrm{p}_{\mathrm{T}}^{\mathrm{J}} / \mathrm{p}_{\mathrm{T}}^{\gamma}\right)$, can be used as a good estimator of the jet fractional $\mathrm{p}_{\mathrm{T}}$ resolution. In real events, the momentum balance in the transverse plane is modified by the presence of additional jets originated from initial or final state radiation. The measurement can be corrected for this effect by extrapolating the measured resolution, in the fractions of photon $\mathrm{p}_{\mathrm{T}}$ carried by the second jet $\mathrm{p}_{\mathrm{T}}$, to the limit of zero secondary jet activity.

To measure the jet energy resolution in a data-driven way, the observable $\sigma\left(\mathrm{p}_{\mathrm{T}}^{\mathrm{Jet}} / \mathrm{p}_{\mathrm{T}}^{\gamma}\right)$ is factorized as

$$
\sigma\left(\mathrm{p}_{\mathrm{T}}^{\mathrm{Jet}} / \mathrm{p}_{\mathrm{T}}^{\gamma}\right)=\sigma\left(\mathrm{p}_{\mathrm{T}}^{\text {Jet }} / \mathrm{p}_{\mathrm{T}}^{\text {ParticleJet }}\right) \oplus \sigma\left(\mathrm{p}_{\mathrm{T}}^{\text {ParticleJet }} / \mathrm{p}_{\mathrm{T}}^{\gamma}\right)
$$

which can be also written as

$$
\sigma(\text { meas })=\sigma(\text { int }) \oplus \sigma(\text { imb })
$$


The first term on the right side of the equation (5.1) is the intrinsic (MC truth) resolution of interest and the second term is the imbalance term arising because of the presence of the secondary jet activities in an event.

\subsubsection{Dataset and Event Selection}

The results I present for the $\gamma+$ jet resolution measurements are based on 36.1 $\mathrm{pb}^{-1}$ of proton-proton collision data collected during 2010 and reconstructed with CMS software. The MC $\gamma+$ jets event sample is generated using PYTHIA6 [46] and processed through the GEANT4 [45] based on a detailed simulation of the CMS detector, which includes the detector response and material.

The $\gamma+$ jet data used in this analysis are collected with different trigger paths based on the $\mathrm{p}_{\mathrm{T}}$ of $\gamma$. The jet resolution is measured in bins of $\gamma \mathrm{p}_{\mathrm{T}}$ that are defined in a such way that each $\mathrm{p}_{\mathrm{T}}$ bin only contains events from a single trigger path. The events with selected trigger paths, summarized in Table 5.2, are used in jet resolution measurements. The $\mathrm{p}_{\mathrm{T}}$ distributions for the leading photons collected with different HLT paths are shown in Figure 5.2.

The $\gamma+$ jet data sample is dominated by a QCD dijet background, in which a jet mimics the photon. To suppress this background, HCAL, ECAL, tracker isolations, and shower shape requirements are applied to the data [60]. To discriminate photons from electrons, events with a track seed in the pixel detector are vetoed.

Events with at least one identified photon (selected with the above isolation parameters) with $\mathrm{p}_{\mathrm{T}}>10 \mathrm{GeV}$ and $|\eta|<1.1$, where the photon and the leading jet 


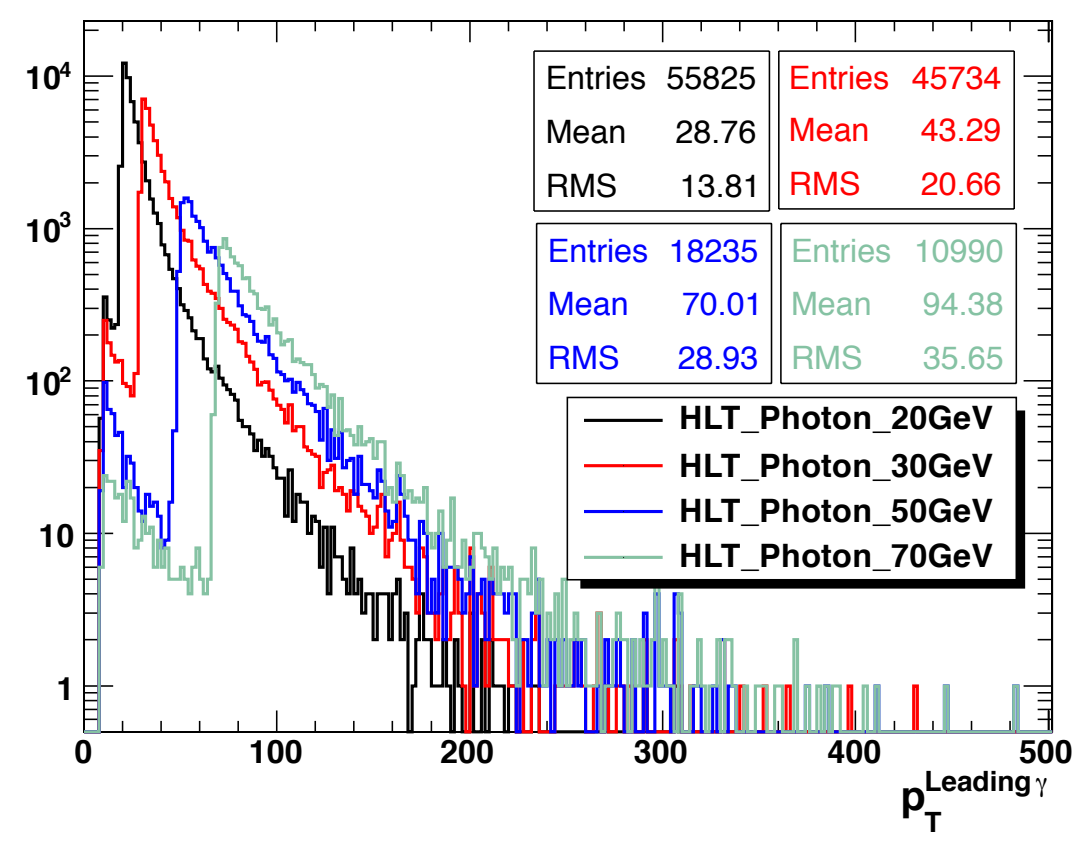

Figure 5.2: Photon $\mathrm{p}_{\mathrm{T}}$ distribution collected with different photon HLT paths.

Table 5.2: Photon HLT paths and corresponding photon $\mathrm{p}_{\mathrm{T}}$

bins used in jet resolution measurements.

\begin{tabular}{|l|l|}
\hline Photon $\mathrm{p}_{\mathrm{T}}(\mathrm{GeV})$ & HLT Paths \\
\hline $22-32$ & HLT_Photon20_L1R \\
$32-52$ & HLT_Photon20_Cleaned_L1R \\
& HLT_Photon30_L1R \\
& HLT_Photon30_L1R_8E29 \\
$52-72$ & HLT_Photon30_Cleaned_L1R \\
& HLT_Photon50_L1R \\
& HLT_Photon50_NoHE_Cleaned_L1R \\
$>72$ & HLT_Photon50_Cleaned_L1R_v1 \\
& HLT_Photon70_NoHE_Cleaned_L1R_v1 \\
& HLT_Photon70_Cleaned_L1R_v1 \\
\hline
\end{tabular}




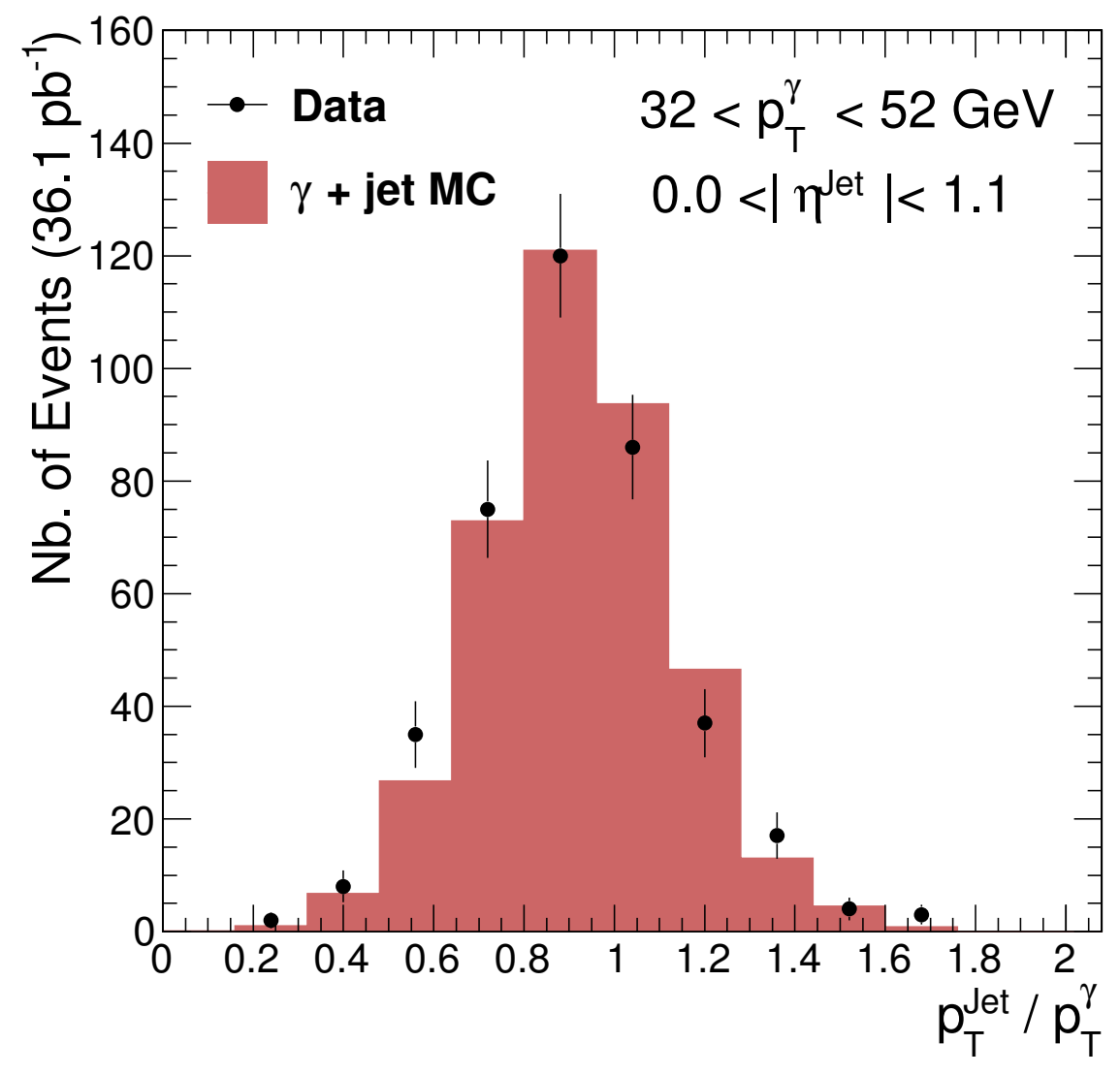

Figure 5.3: $\mathrm{p}_{\mathrm{T}}^{\mathrm{Jet}} / \mathrm{p}_{\mathrm{T}}^{\gamma}$ distribution for $32<\mathrm{p}_{\mathrm{T}}^{\gamma}<52 \mathrm{GeV}$.

are back-to-back $(\mid \Delta \phi(\gamma$, jet1) $\mid>2.7 \mathrm{rad})$ are used to measure jet resolutions.

\subsubsection{Measurement of Resolutions in Data and MC}

After the event selections listed in Section 5.2.1.1, $\mathrm{p}_{\mathrm{T}}^{\mathrm{Jet}} / \mathrm{p}_{\mathrm{T}}^{\gamma}$ distributions are measured in data and MC. The MC and data comparison for the $\mathrm{p}_{\mathrm{T}}^{\mathrm{Jet}} / \mathrm{p}_{\mathrm{T}}^{\gamma}$ measurement for the 32-52 GeV $\gamma$ photon $\mathrm{p}_{\mathrm{T}}$ bin is shown in Figure 5.3. Distributions measured in the $\mathrm{MC}$ well predict data.

To study the additional jet activity in $\gamma+$ jet events I measure the resolutions 


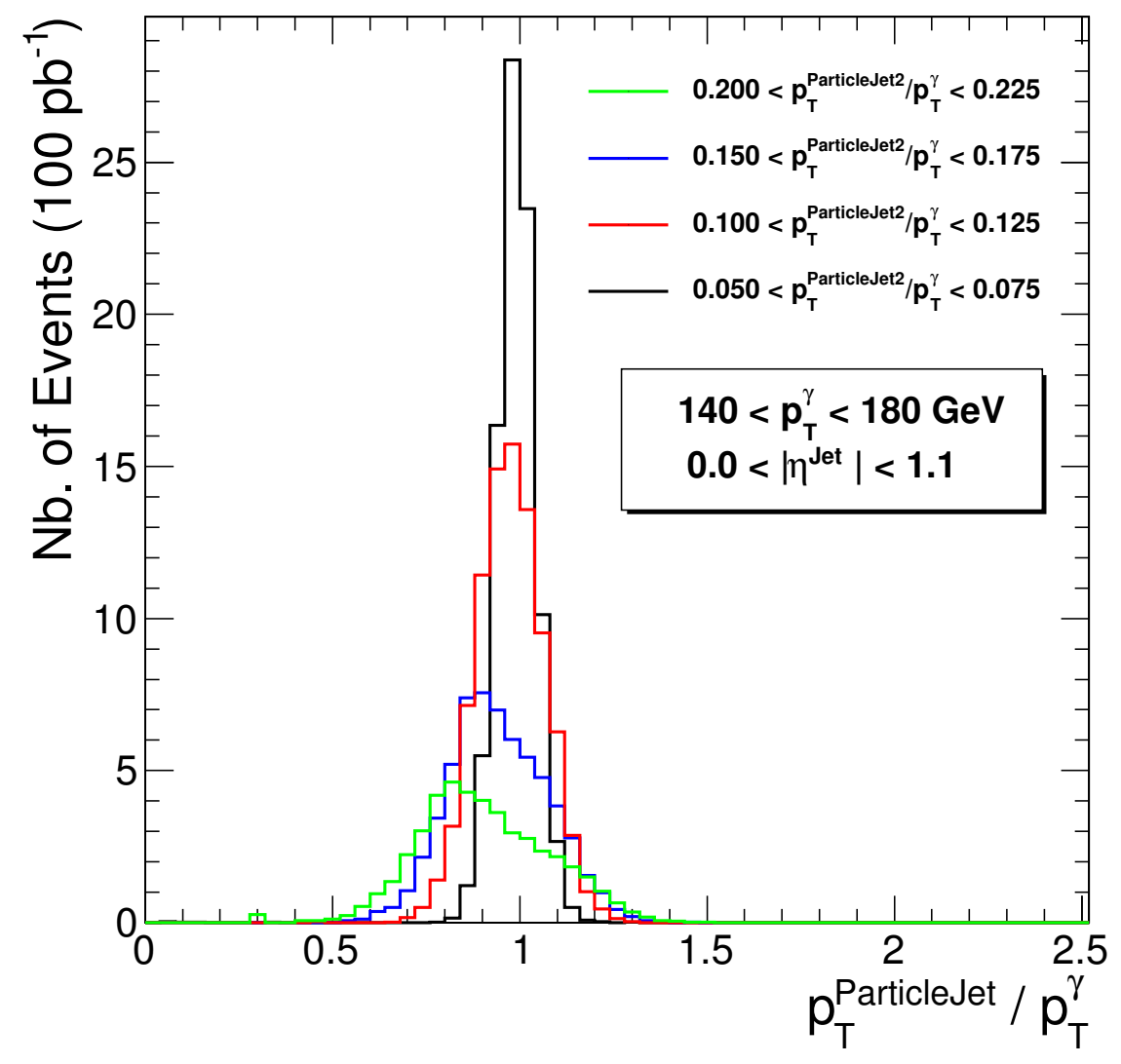

Figure 5.4: Left: Imbalance $\left(\mathrm{p}_{\mathrm{T}}^{\text {ParticleJet }} / \mathrm{p}_{\mathrm{T}}^{\gamma}\right)$ distributions for different bins of $\mathrm{p}_{\mathrm{T}}^{\text {ParticleJet2 }} / \mathrm{p}_{\mathrm{T}}^{\gamma}$ and for $140<\mathrm{p}_{\mathrm{T}}<180 \mathrm{GeV}$.

(measured, intrinsic, imbalance) in bins of $\mathrm{p}_{\mathrm{T}}^{\text {Jet2 }} / \mathrm{p}_{\mathrm{T}}^{\gamma}$. Figure 5.4 shows the imbalance distributions for different bins of $\mathrm{p}_{\mathrm{T}}^{\text {ParticleJet } 2} / \mathrm{p}_{\mathrm{T}}^{\gamma}$ in $\mathrm{MC}$. The effect of reducing the second jet activity is clearly visible as a narrowing of the spread of the distributions.

The resolutions I measure for the $52-72 \mathrm{GeV} \gamma \mathrm{p}_{\mathrm{T}}$ bin in the bins of $\mathrm{p}_{\mathrm{T}}^{\mathrm{Jet} 2} / \mathrm{p}_{\mathrm{T}}^{\gamma}$ are shown in Figure 5.5. Blue filled circles represent the intrinsic resolutions of reconstructed jets measured as the width of $\mathrm{p}_{\mathrm{T}}^{\mathrm{Jet}} / \mathrm{p}_{\mathrm{T}}^{\text {ParticleJet }}$ for which reconstructed jets are 
matched to particle jets within a radius of $\Delta \mathrm{R}=0.1\left(\Delta \mathrm{R} \equiv \sqrt{(\Delta \eta)^{2}+(\Delta \phi)^{2}}\right)$. The variation of $\Delta \mathrm{R}$ matching can affect resolution measurements. I performed a study to evaluate this effect (Appendix $\mathrm{B}$ ). The study showed that varying $\Delta \mathrm{R}$ matching from 0.10 to $0.15,0.20,0.25$, and 0.30 does not have a significant effect on measured resolutions. The measured resolution (width of $\mathrm{p}_{\mathrm{T}}^{\mathrm{Jet}} / \mathrm{p}_{\mathrm{T}}^{\gamma}$ ) is shown with red open circles for data and with violet filled circles for MC. The distribution shown with black filled circles is the MC imbalance resolution (width of $\mathrm{p}_{\mathrm{T}}^{\text {ParticleJet }} / \mathrm{p}_{\mathrm{T}}^{\gamma}$ ). Figure 5.5 shows that measured and imbalance resolutions are affected by the additional jet activities in the events, whereas the intrinsic resolution is independent of any other activity in an event.

To obtain the Data/MC ratio, first the ratio of the intrinsic resolutions measured in data and MC is obtained, then it is extrapolated to zero as a function of $\mathrm{p}_{\mathrm{T}}^{\mathrm{jet} 2} / \mathrm{p}_{\mathrm{T}}^{\gamma}$. The $\sigma(\mathrm{imb})$ component measured from MC is assumed to be the same in the data. Intrinsic resolutions in data and MC are calculated by subtracting the MC imbalance component from $\sigma\left(\mathrm{p}_{\mathrm{T}}^{\mathrm{Jet}} / \mathrm{p}_{\mathrm{T}}^{\gamma}\right)$ in quadrature for various bins of $\mathrm{p}_{\mathrm{T}}^{\mathrm{jet} 2} / \mathrm{p}_{\mathrm{T}}^{\gamma}$. By following equation (5.2), intrinsic resolutions for data and MC can be written as

$$
\begin{aligned}
\left(\sigma_{\text {Data }}(\text { int })\right)^{2} & =\left(\sigma_{\text {Data }}(\text { meas })\right)^{2}-\left(\sigma_{\mathrm{MC}}(\text { imb })\right)^{2} \\
\left(\sigma_{\mathrm{MC}}(\text { int })\right)^{2} & =\left(\sigma_{\mathrm{MC}}(\text { meas })\right)^{2}-\left(\sigma_{\mathrm{MC}}(\text { imb })\right)^{2}
\end{aligned}
$$

The imbalance component subtracted measured resolution $(\sigma(\mathrm{int}))$ is expected to be independent of $\mathrm{p}_{\mathrm{T}}^{\mathrm{Jet} 2}$ in simulations, and therefore it is fitted with a zero degree polynomial before the Data/MC ratio is calculated. The measured (left) and intrinsic 


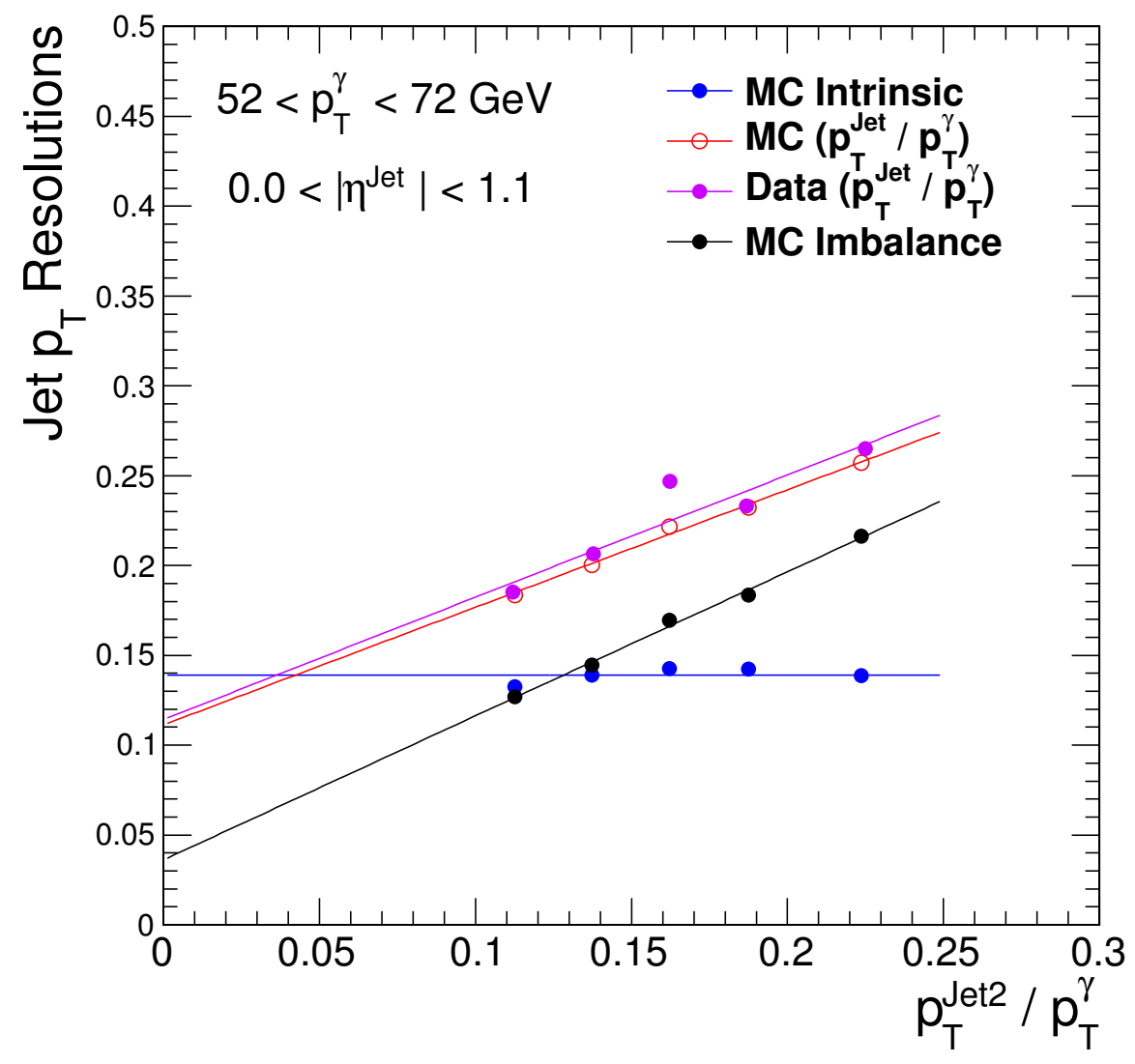

Figure 5.5: Components of jet resolution functions in $140-180 \mathrm{GeV}$ photon $\mathrm{p}_{\mathrm{T}}$ bin as a function of $\mathrm{p}_{\mathrm{T}}^{\mathrm{Jet} 2} / \mathrm{p}_{\mathrm{T}}^{\gamma}$ are shown for data and $\mathrm{MC}$.

(right) resolutions for data and MC are shown in Figure 5.6 for the $90-110 \mathrm{GeV} \gamma$ $\mathrm{p}_{\mathrm{T}}$ bin.

One of the main contribution to the uncertainty in measuring resolutions comes from the extrapolation procedure used to obtain the limit in the case of zero secondary jet activity. To address this issue, two fit ranges are used for extrapolation depending on the photon $\mathrm{p}_{\mathrm{T}}$ bin used. For low momentum $\mathrm{p}_{\mathrm{T}}$ bins like $22-32,32-52$ or 

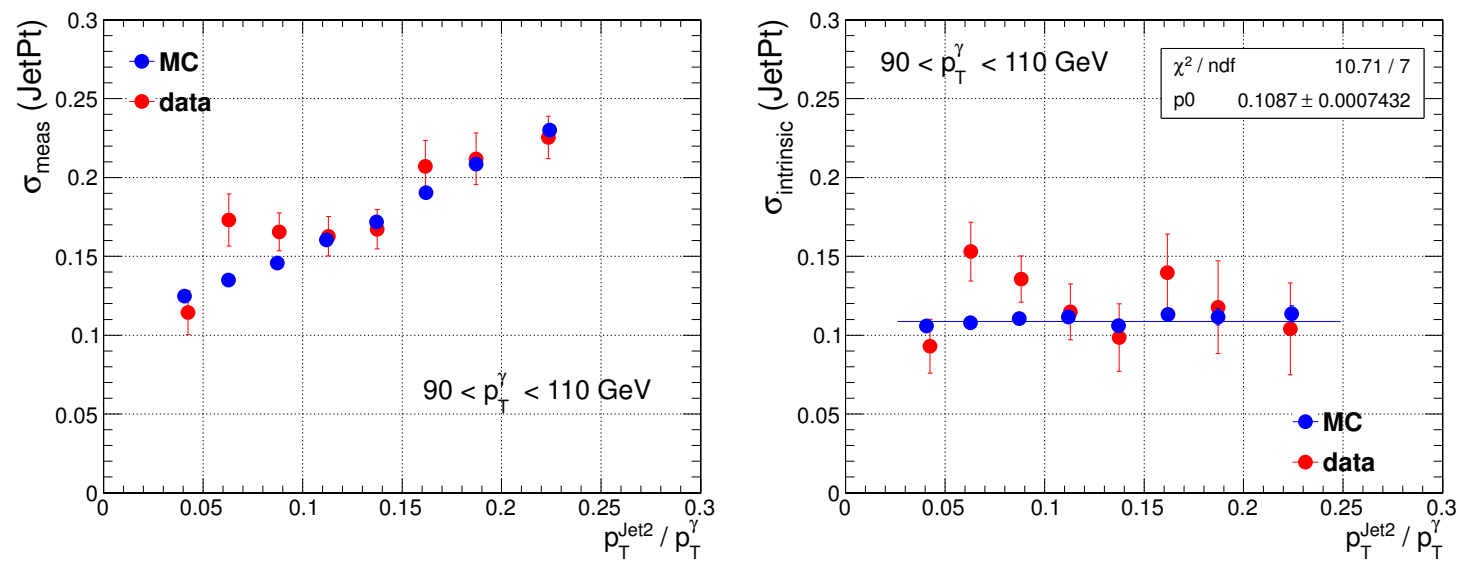

Figure 5.6: Measured (left) and intrinsic (right) resolutions in Data (red) and MC (blue) in the $90-110 \mathrm{GeV}$ photon $\mathrm{p}_{\mathrm{T}}$ bin for $0.0<|\eta|<1.1$ region.

52-72 GeV, resolutions measured at lower values of $\mathrm{p}_{\mathrm{T}}^{\mathrm{Jet} 2} / \mathrm{p}_{\mathrm{T}}^{\gamma}$ fractions are biased due to reconstruction inefficiency of jets and higher uncertainties in jet energy corrections. Similarly for higher $\mathrm{p}_{\mathrm{T}}$ bins $(100-140 \mathrm{GeV}$ or higher), including resolutions measured at higher values of $\mathrm{p}_{\mathrm{T}}^{\mathrm{Jet}} / \mathrm{p}_{\mathrm{T}}^{\gamma}$ fractions result in another bias due to a larger deviation from the two body process. Therefore for each $\mathrm{p}_{\mathrm{T}}$ bin used in this study, two fit regions are chosen by including or excluding lower/higher values of second jet $\mathrm{p}_{\mathrm{T}}$ fractions. The left and right columns in Figures 5.7 and 5.8 show the intrinsic resolutions measured in Data and MC for lower and higher extrapolation regions.

Figures 5.9 and 5.10 show the ratio of measured intrinsic resolutions in two different extrapolation regions for the photon $\mathrm{p}_{\mathrm{T}}$ bins I used in this analysis. The nominal Data/MC ratio is taken to be the average of the two cases for the given photon $\mathrm{p}_{\mathrm{T}}$ bin. 

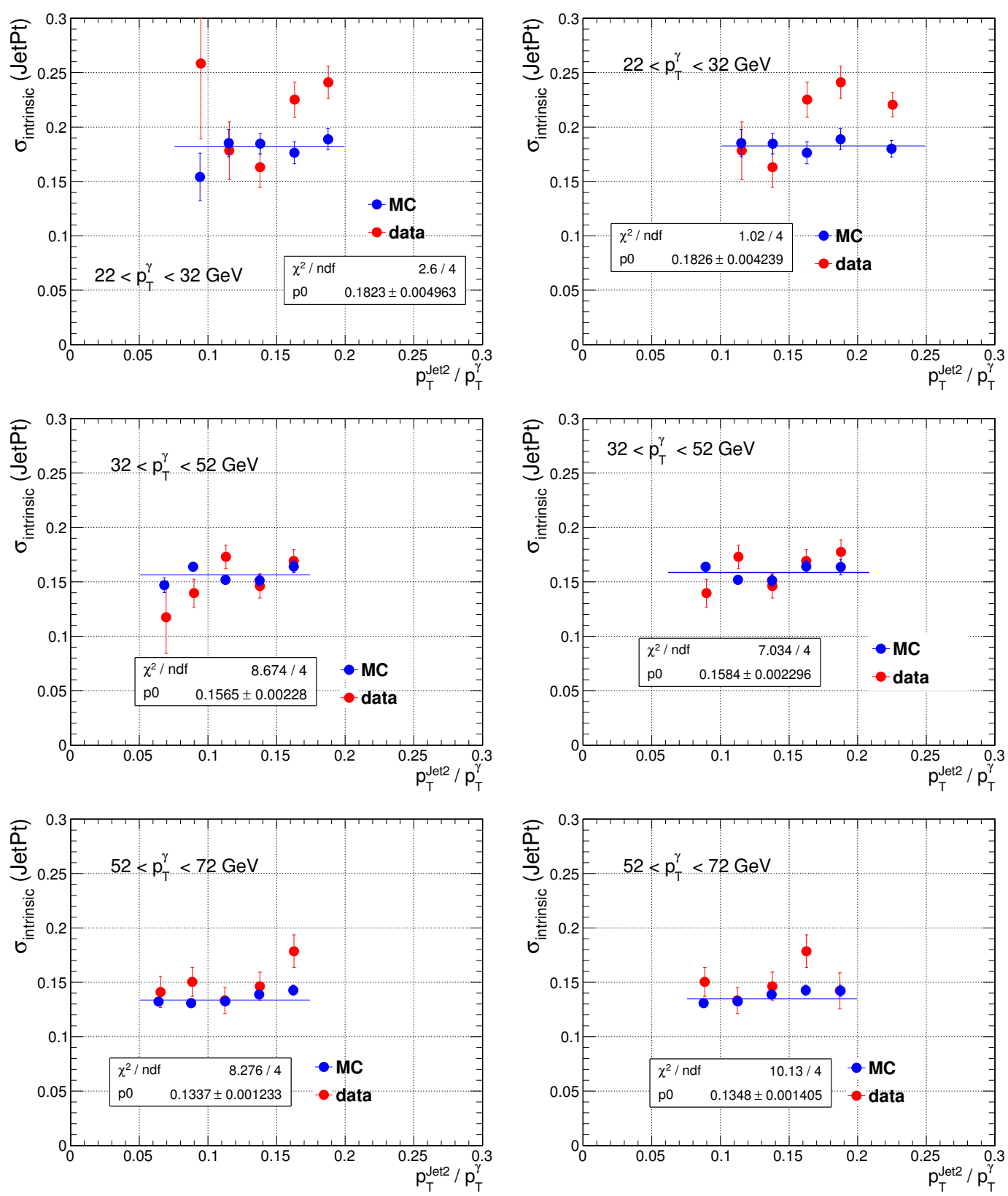

Figure 5.7: Intrinsic resolutions measured in Data (red) and MC (blue) for the photon $\mathrm{p}_{\mathrm{T}}$ bins $(22-32),(32-52)$, and $(52-72) \mathrm{GeV}$ for the $|\eta|<1.1$ region. Distributions are shown for lower (left) and higher (right) extrapolation regions. 

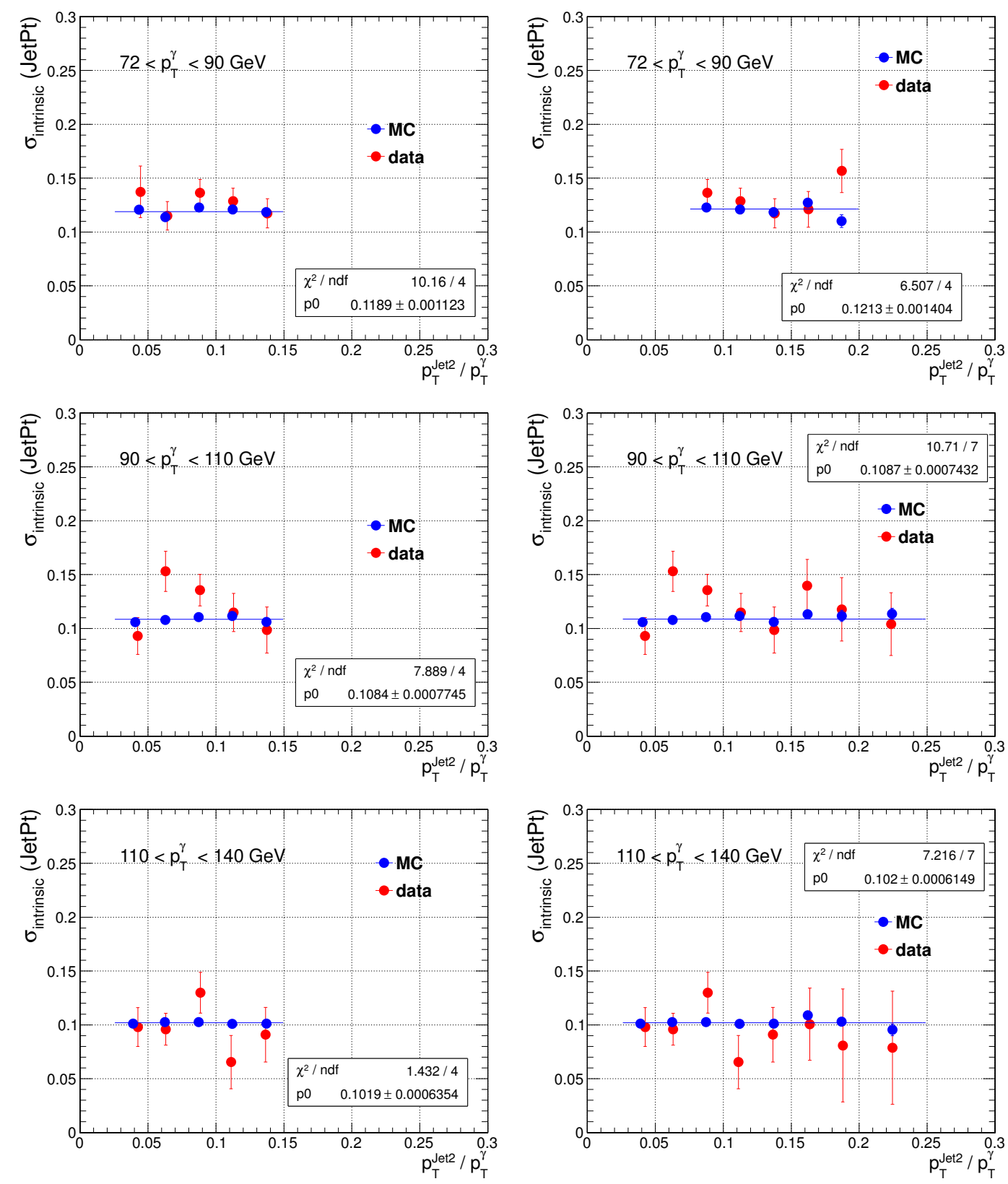

Figure 5.8: Intrinsic resolutions measured in Data (red) and MC (blue) for the photon $\mathrm{p}_{\mathrm{T}}$ bins $(72-90),(90-110)$, and $(110-140) \mathrm{GeV}$ for the $|\eta|<1.1$ region. Distributions are shown for lower (left) and higher (right) extrapolation regions. 

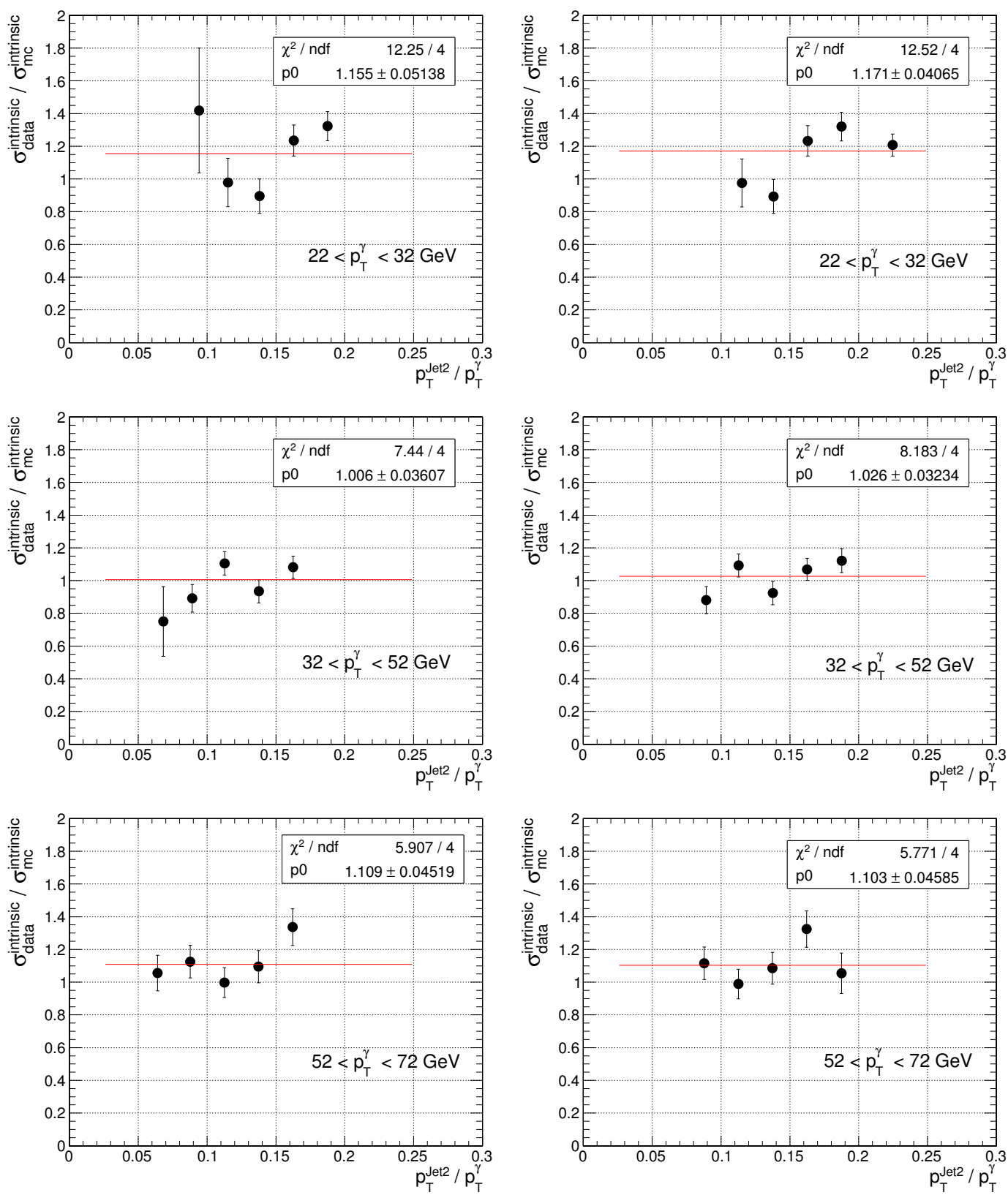

Figure 5.9: Ratio of intrinsic resolutions measured in Data and MC for the photon $\mathrm{p}_{\mathrm{T}}$ bins $(22-32),(32-52)$, and $(52-72) \mathrm{GeV}$ for the $|\eta|<1.1$ region. Distributions are shown for lower (left) and higher (right) extrapolation regions. 

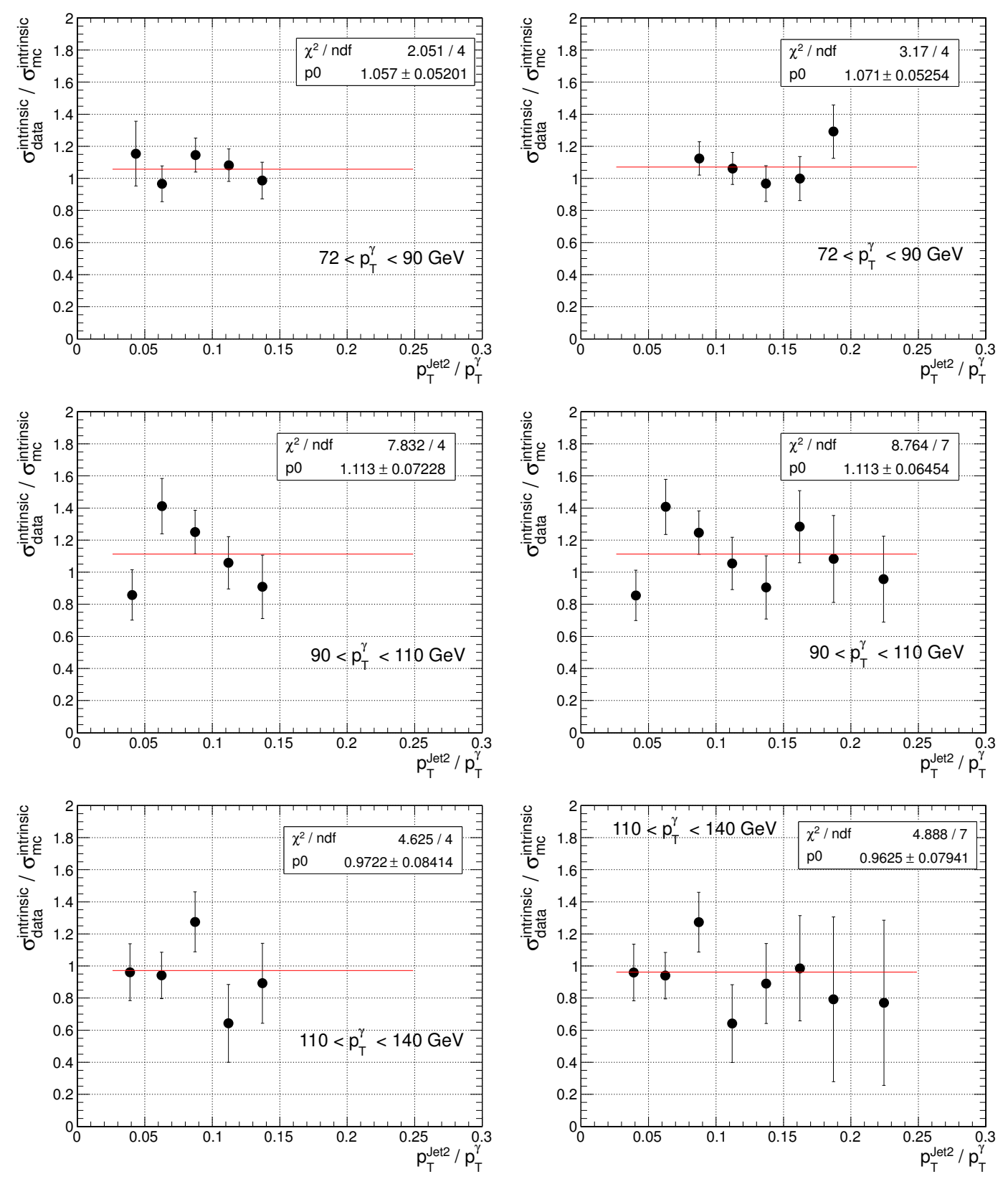

Figure 5.10: Ratio of intrinsic resolutions measured in Data and $\mathrm{MC}$ for the photon $\mathrm{p}_{\mathrm{T}}$ bins $(72-90),(90-110)$, and $(110-140) \mathrm{GeV}$ for the $|\eta|<1.1$ region. Distributions are shown for lower (left) and higher (right) extrapolation regions. 
A summary of the measured Data/MC ratio of intrinsic resolutions in the various $\eta$ regions of the detector is shown in Figure 5.11. The measured ratios are consistent with being independent of $\mathrm{p}_{\mathrm{T}}$ and fitted with a zero degree polynomial.

\subsubsection{Estimation of non-Gaussian Component}

To estimate the non-Gaussian component, first the resolutions in the MC sample are stretched according to the measured Data/MC ratio. To stretch measured

resolutions in the $\mathrm{MC}, \mathrm{p}_{\mathrm{T}}^{\mathrm{Jet}}$ is modified by the fractional difference in the intrinsic resolution measured in data and $\mathrm{MC}$ as follow

$$
\mathrm{p}_{\mathrm{T}}^{\mathrm{Jet}}=\mathrm{p}_{\mathrm{T}}^{\mathrm{Jet}}+\left(\mathrm{p}_{\mathrm{T}}^{\text {Jet }}-\mathrm{p}_{\mathrm{T}}^{\text {ParticleJet }}\right) \times\left(\frac{\sigma_{\text {intr }}^{\text {Data }}}{\sigma_{\text {intr }}^{\mathrm{MC}}}-1\right)
$$

After distributions in the MC are modified, the resolutions measured in data and MC are fitted with a Gaussian function and the number of events outside the $2.5 \sigma$ range is counted in the bins of $\mathrm{p}_{\mathrm{T}}^{\gamma}$ to compare resolution tails. The measured resolutions in data and MC for the various photon $\mathrm{p}_{\mathrm{T}}$ bins are shown in Figure 5.12.

By using the event count outside the $2.5 \sigma$ range, the Data/MC ratio for the number of tail events is obtained for different $\mathrm{p}_{\mathrm{T}}^{\gamma}$ bins as shown in Figure 5.13. The event statistics come from the $2010 \gamma+$ jet data do not allow us to measure the resolution tails with precision, but a constant fit to the ratio is consistent within the errors with the study using a dijet sample, and provides a cross check [60]. 


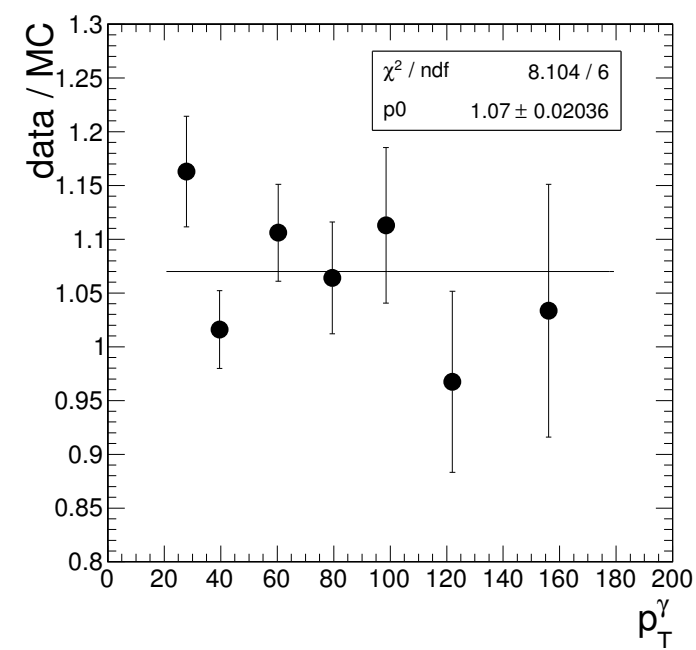

(a) $0.0<\left|\eta^{\text {Jet }}\right|<1.1$

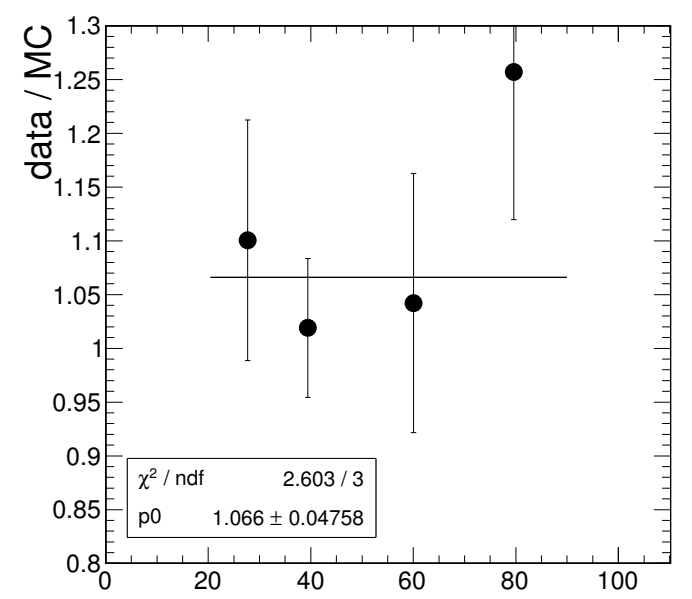

(c) $1.7<\left|\eta^{\mathrm{Jet}}\right|<2.3$

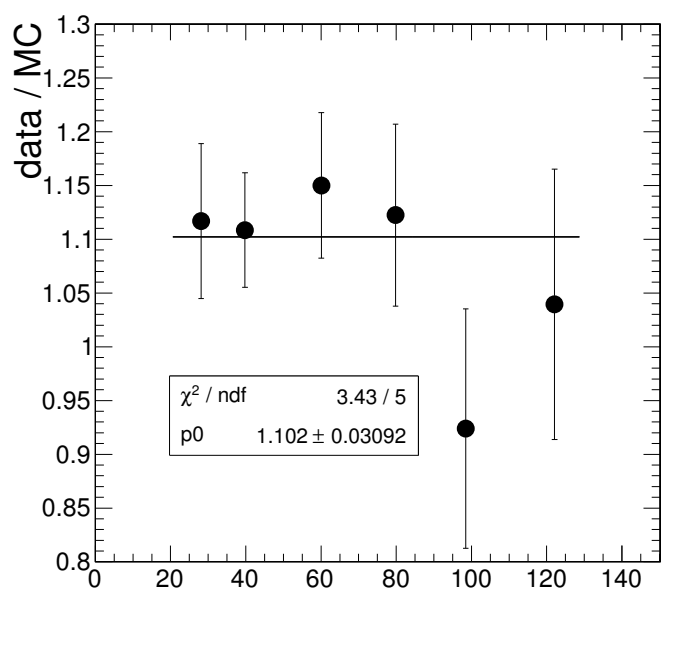

(b) $1.1<\left|\eta^{\text {Jet }}\right|<1.7$

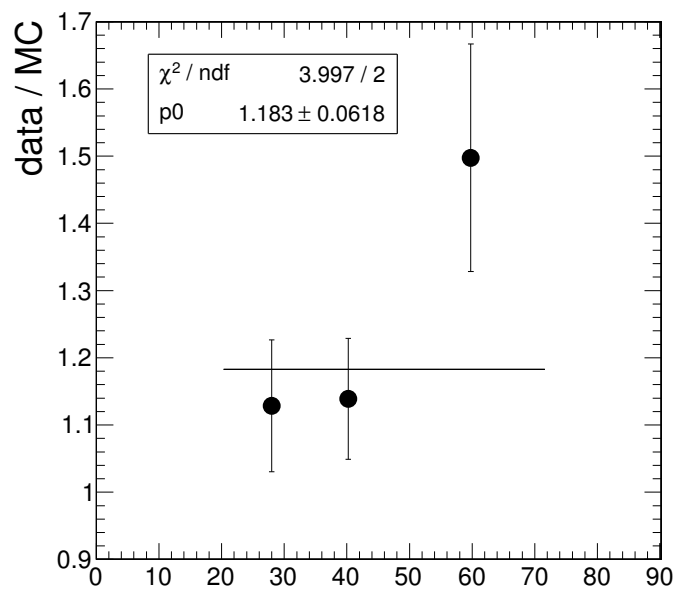

(d) $2.3<\left|\eta^{\text {Jet }}\right|<3.5$

Figure 5.11: Extrapolated ratio of the intrinsic resolution measured in data and MC for the various bins of jet $\eta$. 

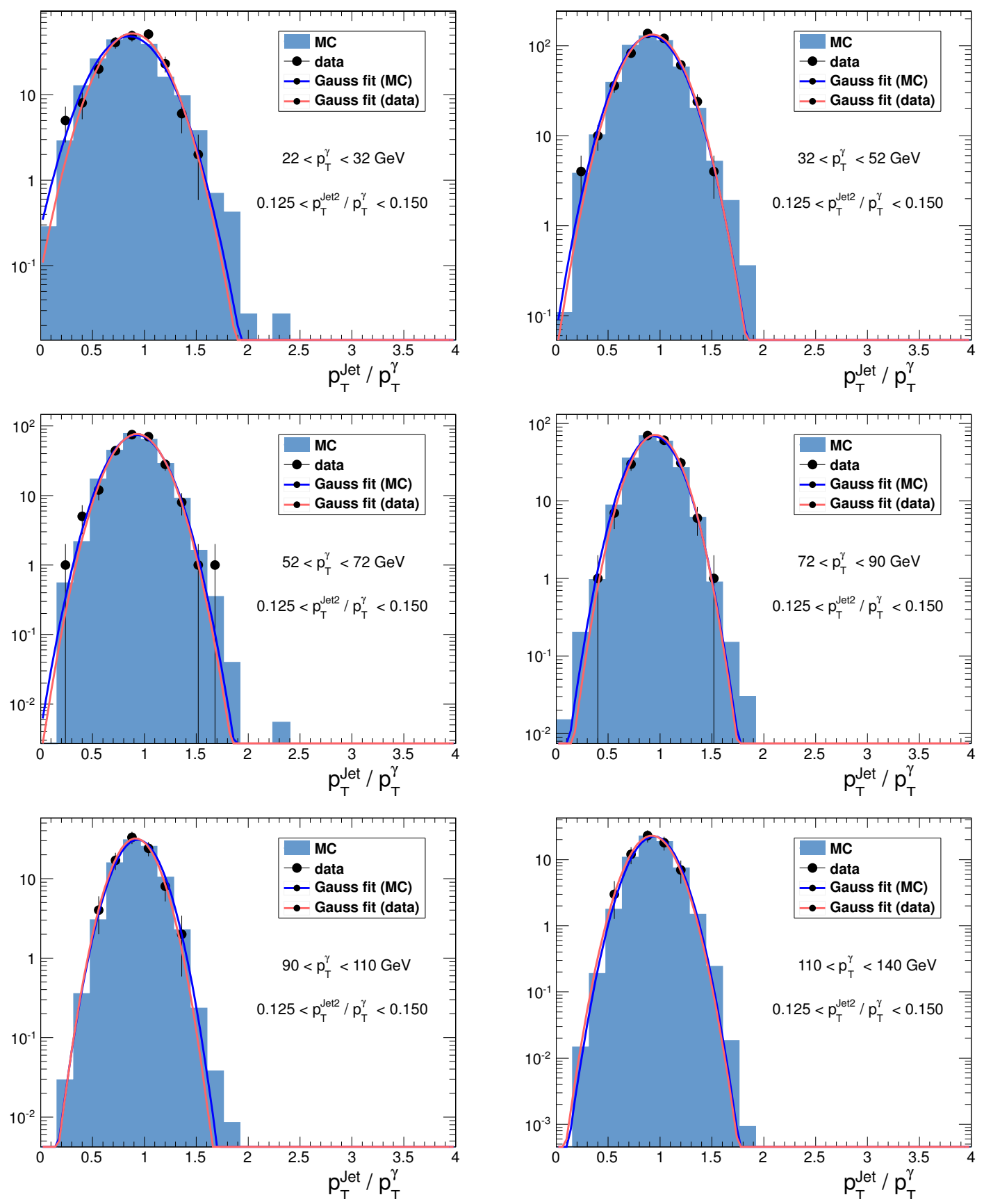

Figure 5.12: Measured jet $\mathrm{p}_{\mathrm{T}}$ resolution functions in $\mathrm{MC}$ and data for different photon $\mathrm{p}_{\mathrm{T}}$ bins for $|\eta|<1.1$. 


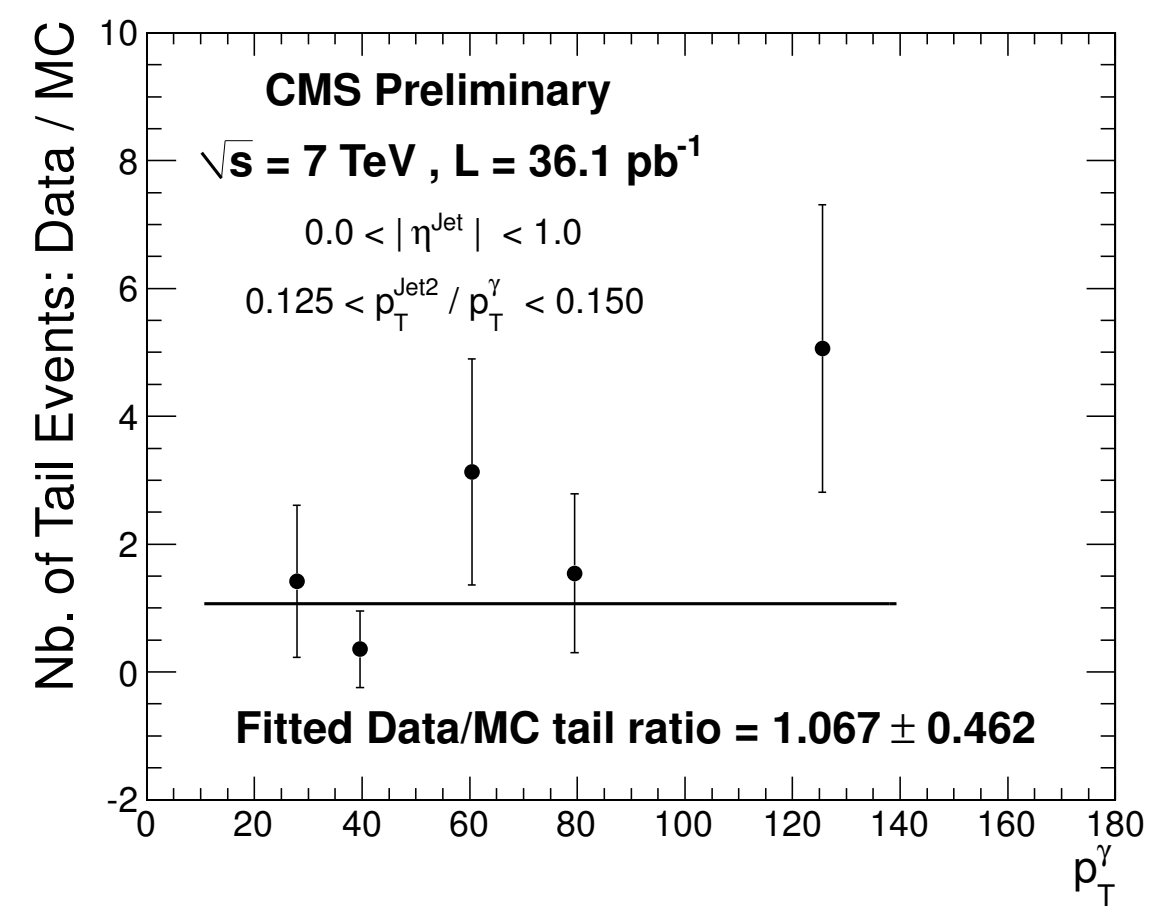

Figure 5.13: Ratio of the number of tail events in data and $\mathrm{MC}$ vs $\mathrm{p}_{\mathrm{T}}^{\gamma}$.

\subsubsection{Dijet Measurements}

The dijet asymmetry method is used to measure the full resolution function from the dijet sample. The dijet asymmetry is calculated as $\left(p_{T}^{\text {Jet } 1}-p_{T}^{\text {Jet2 }}\right) /\left(p_{T}^{\text {Jet } 1}+\right.$ $\left.p_{T}^{\text {Jet2 }}\right)$ with a random ordering of the two highest $\mathrm{p}_{\mathrm{T}}$ jets. The method measures the resolution as a function of jet $\eta$ and $\mathrm{p}_{\mathrm{T}}$. Since the momentum balance in the transverse plane is destroyed by the additional jet activities in the events, an extrapolation to no additional jet activity is performed [44].

The Data/MC ratio from the photon+jet and dijet samples are compared in Figure 5.14 as a function of jet $\mathrm{p}_{\mathrm{T}}$ in the barrel region $(|\eta|<1.1)$. There is a good 


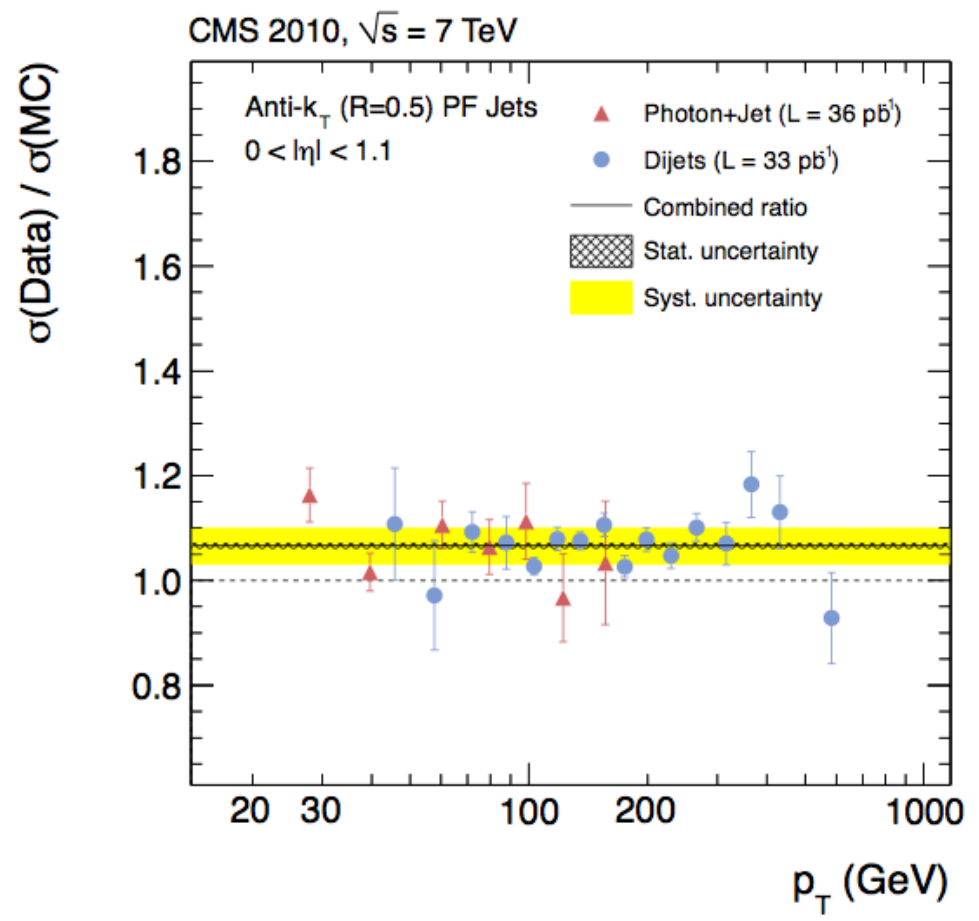

Figure 5.14: The ratio of jet $\mathrm{p}_{\mathrm{T}}$ resolutions in data and $\mathrm{MC}$ samples versus jet $\mathrm{p}_{\mathrm{T}}$, in $|\eta|<1.1$, from dijet and $\gamma+$ jet samples; a combined fit to both data sets is also shown [60].

agreement between dijet and $\gamma+$ jet measurements.

The Data/MC ratio measured with the dijet sample is calculated separately for Gaussian and non-Gaussian parts and the Gaussian component is combined with $\gamma+$ jet results. The combined Data/MC ratio for the various $\eta$ regions of the detector is shown in Figure 5.15. Table 5.3 summarizes Data/MC ratios measured from dijet and $\gamma+$ jet samples with statistical and systematical uncertainties. Details of systematical uncertainty measurements are described in Section 5.2.3. Both methods $(\gamma+$ jet and 


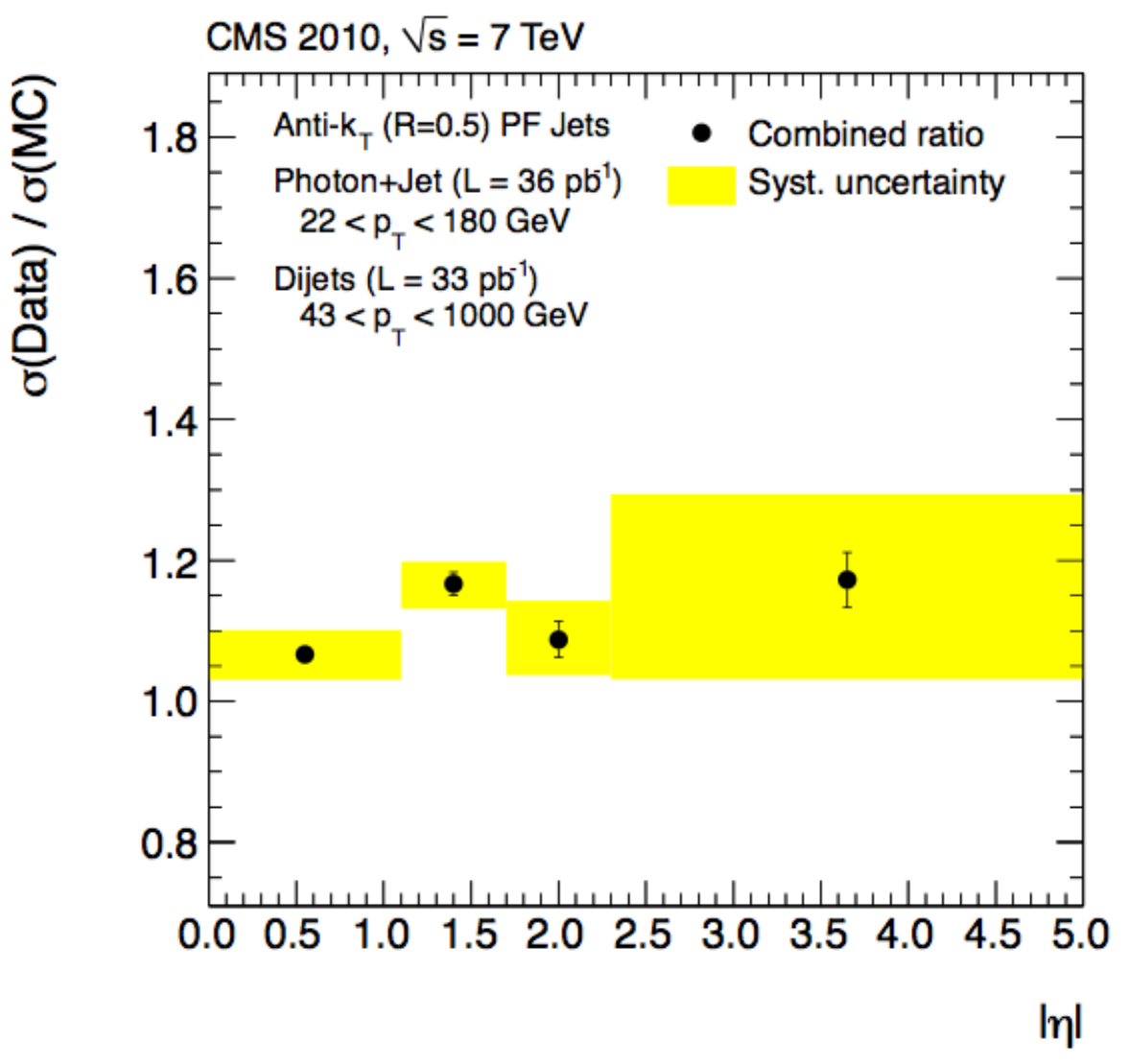

Figure 5.15: Results from the combination fits versus jet $\mathrm{p}_{\mathrm{T}}$ in various $\eta$ ranges (right) [60].

dijet) measure the core of the Gaussian resolution 5-10\% higher in data compared to simulations. Since no significant non-Gaussian tails are observed in $\gamma+$ jet events, the correction factors to the jet resolution tails are derived by using the dijet sample. Figure 5.16 shows the Data/MC correction factor measured from the dijet sample for the jet resolution tails in the barrel region.

The nominal resolution functions are obtained by first modifying the Gaussian 
Table 5.3: Ratio of the resolutions measured in data and MC for different jet $\eta$ ranges using the unbinned likelihood fits in the dijet and $\mathrm{p}_{\mathrm{T}}$ balance method in $\gamma+$ jet samples.

\begin{tabular}{|c|c|c|}
\hline$|\eta|$ & Data/MC ratio in dijet & Data/MC ratio in $\gamma+$ jet \\
\hline $0.0-1.1$ & $1.066 \pm 0.007+0.074-0.082$ & $1.07 \pm 0.020+0.024-0.033$ \\
\hline $1.1-1.7$ & $1.191 \pm 0.019+0.064-0.062$ & $1.10 \pm 0.031+0.031-0.039$ \\
\hline $1.7-2.3$ & $1.096 \pm 0.030+0.089-0.085$ & $1.07 \pm 0.048+0.056-0.047$ \\
\hline $2.3-3.5$ & $1.166 \pm 0.050+0.198-0.199$ & $1.18 \pm 0.062+0.043-0.072$ \\
\hline
\end{tabular}

Results are given with statistical uncertainties from the fit and the upper and lower systematical uncertainties [60].

core of the MC truth resolutions, measured from the QCD multijet sample, with the correction factors derived from $\gamma+$ jet and dijet samples. Then both the lower and upper tails of the MC truth resolutions are scaled by the tail corrections factors derived from dijet samples. The nominal resolutions are used for smearing seed events in the inclusive data sample.

\subsubsection{Systematic Uncertainty Measurements}

The systematic uncertainties given in Table 5.3 for the dijet asymmetry methods are evaluated using the QCD MC samples. The following items are studied to evaluate the systematic uncertainty of the Data/MC ratio measured from the dijet sample:

- particle level dijet cross section

- jet energy scale 


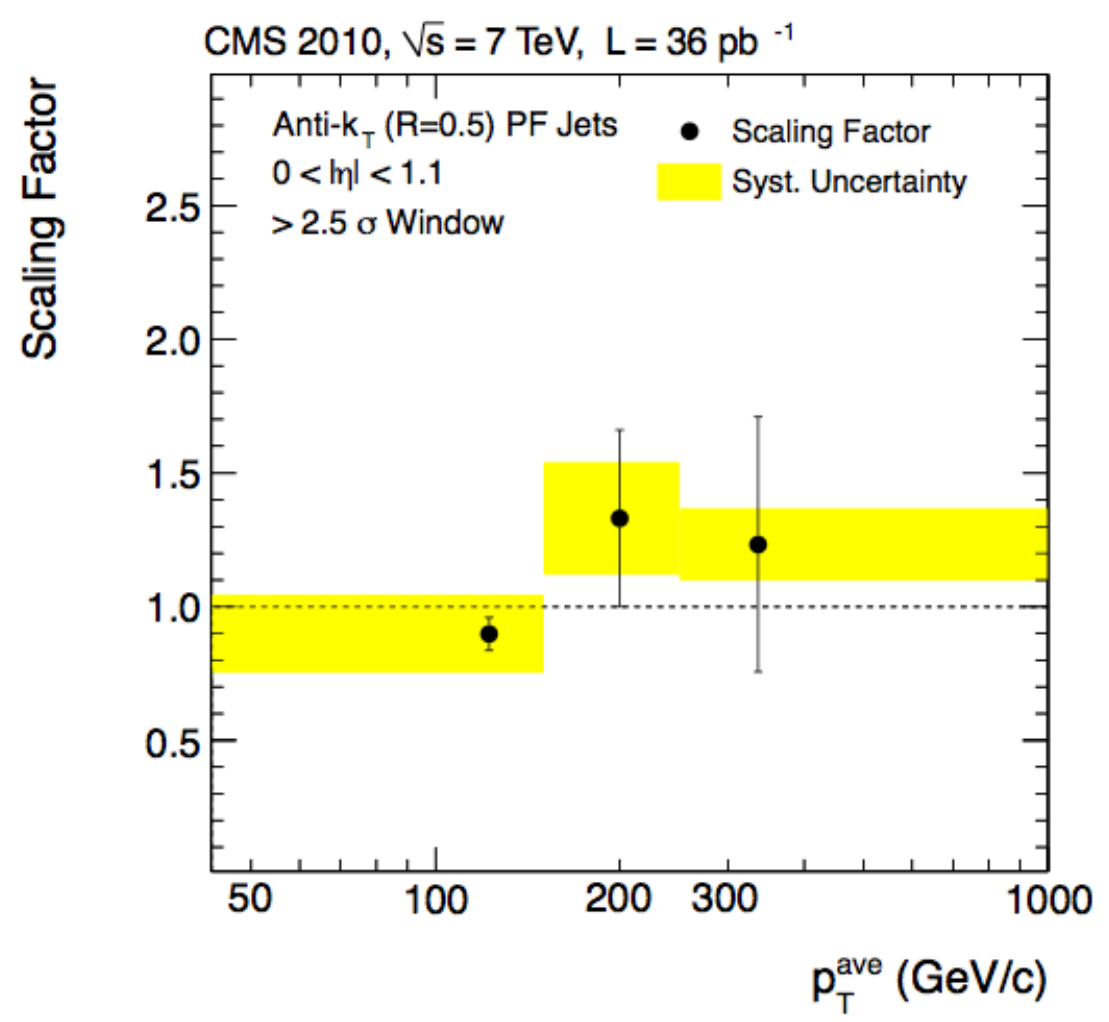

Figure 5.16: The Data/MC correction factor for the jet resolution tails observed in dijet samples for barrel region, using the range greater than $2.5 \sigma$ window [60].

- correction for additional hadronic activity

- hadronization and out-of-cone correction

- non-closure in the MC sample.

The contribution from each item is accepted to be uncorrelated and the final systematic uncertainties listed in Table 5.3 are calculated as the quadrature sum of the individual components. 
To complete the Gaussian core jet resolution measurements from the $\gamma+$ jet sample, I studied the following systematic uncertainties on the measured Data/MC ratio:

1. Variation of the extrapolation fit range.

2. Effect of $\Delta \phi(\gamma$, jet1) requirement.

3. Uncertainty of jet energy corrections.

4. Particle level imbalance.

5. Flavor difference between $\gamma+$ jet and dijet events.

6. Pileup subtraction.

Since the Data/MC ratio of intrinsic resolutions is our main measurement, I studied how much each item listed above can change the measured Data/MC ratio. For each item, the Data/MC ratio is recalculated and the relative difference between the new and nominal ratios is used in systematic uncertainty assignments.

\subsubsection{Uncertainty Due to Extrapolation Fit Range}

As mentioned in Section 5.2.1.2, the extrapolation procedure for obtaining the limit in the case of zero secondary jet activity is one of the systematic uncertainties in measuring the Data/MC ratio. I studied this systematic effect by choosing two different extrapolation regions for each $\gamma \mathrm{p}_{\mathrm{T}}$ bin used in this analysis. In the first extrapolation region, the lower $\mathrm{p}_{\mathrm{T}}^{\mathrm{Jet} 2} / \mathrm{p}_{\mathrm{T}}^{\gamma}$ fraction points are included in the fit, whereas in the second region, higher fraction points are included in the fit procedure. I measured the extrapolated Data/MC ratio of intrinsic resolutions for the two fit regions, 


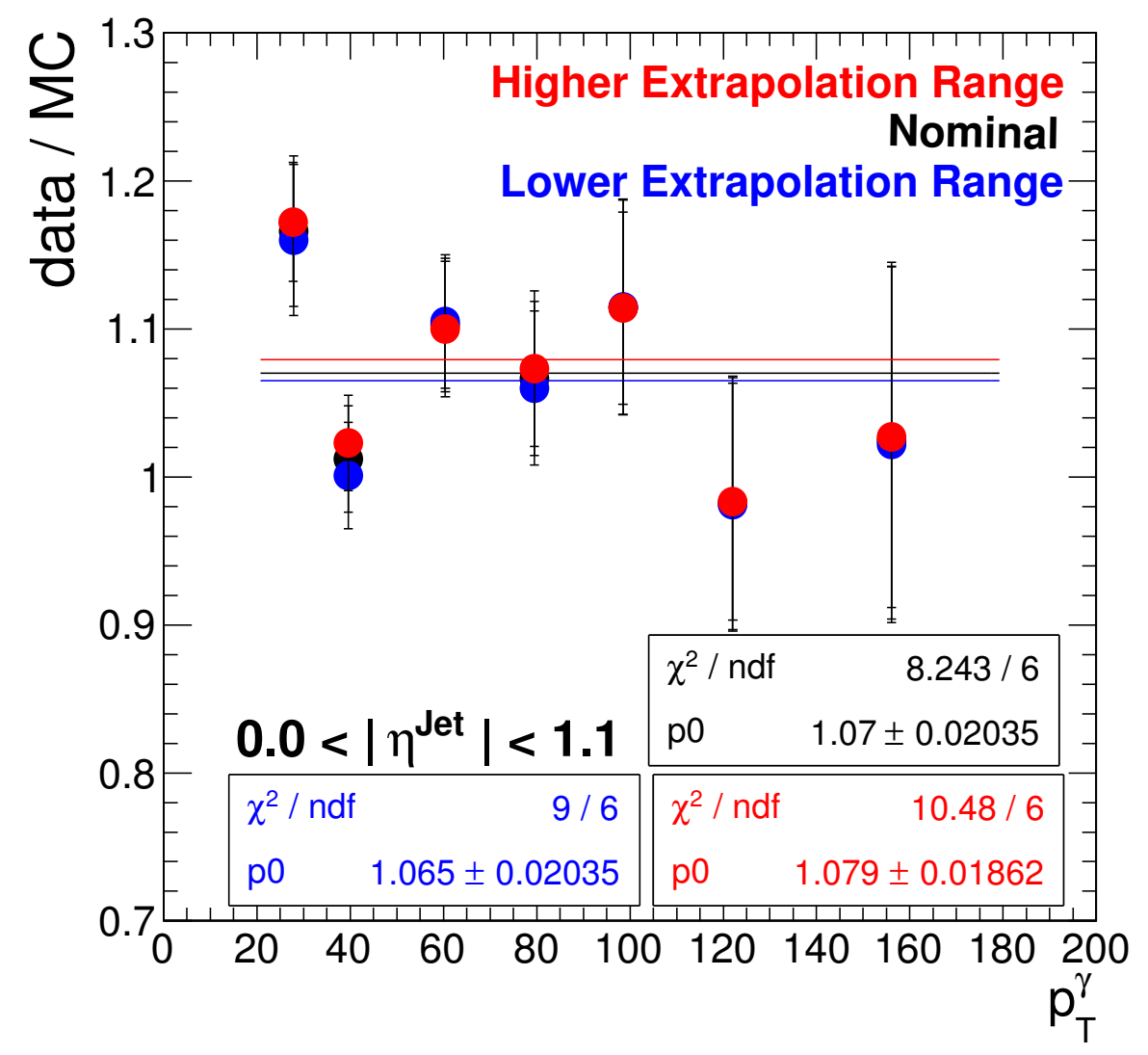

Figure 5.17: Data/MC ratio for nominal (black), lower extrapolation range (blue), and higher extrapolation range (red) measurements for the $0.0<|\eta|<1.1$ region.

and then used relative differences between the two Data/MC ratios to define the uncertainty due to the extrapolation fit range. Figure 5.17 shows the nominal Data/MC ratios together with the ratios I calculated for lower and higher extrapolation.

\subsubsection{Uncertainty Due to $\Delta \phi(\gamma$, jet1) Requirement}

To obtain back-to-back $\gamma+$ jet events in resolution measurements I only used events where $\Delta \phi$ between the leading jet and $\gamma$ is greater than 2.7 radians. To 
study the effect of this requirement on the measured Data/MC ratio, I measured intrinsic resolutions in the MC with two different $\Delta \phi$ selections, which are marked as $\mathrm{MC}(1)$ and $\mathrm{MC}(2)$, by following the method described in Section 5.2.1.2, then calculated their ratio for the pseudorapidity ranges of $(0.0-1.1),(1.1-1.7),(1.7-2.3)$ and (2.3-3.5). Figure 5.18 shows the $\mathrm{MC}(1) / \mathrm{MC}(2)$ ratio for the barrel region where I measured $\mathrm{MC}(1)$ with $\mid \Delta \phi(\gamma$, jet1) $\mid>2.1$ and $\mathrm{MC}(2)$ with $\mid \Delta \phi(\gamma$, jet1) $\mid>2.7$. Since the deviation from 1 on the measured $\mathrm{MC}(1) / \mathrm{MC}(2)$ ratio is less than $1 \%$, the effect of the $\Delta \phi$ requirement is not taken into account in systematic error calculations.

\subsubsection{Uncertainty Due to Jet Energy Corrections}

The jet energy correction (JEC) measurement is one of the main systematic uncertainties affecting the Data/MC calculations. To determine the systematic uncertainty due to JEC, I varied the jet energy corrections I applied on the reconstructed jets up and down with the official uncertainties provided by the CMS JEC group [40].

By following the procedure explained in Section 5.2.1.2, I recalculated the Data/MC ratios with new JECs and measured the relative differences between the two ratios (JEC up and JEC down). Figure 5.19 shows how the Data/MC ratio of intrinsic resolutions changes if JECs are varied by nominal uncertainties for the barrel region. I assigned the relative difference between JEC up (1.08) and JEC down (1.044) Data/MC ratios as a systematic uncertainty and repeated the same procedure for the other eta bins I used in this study. 


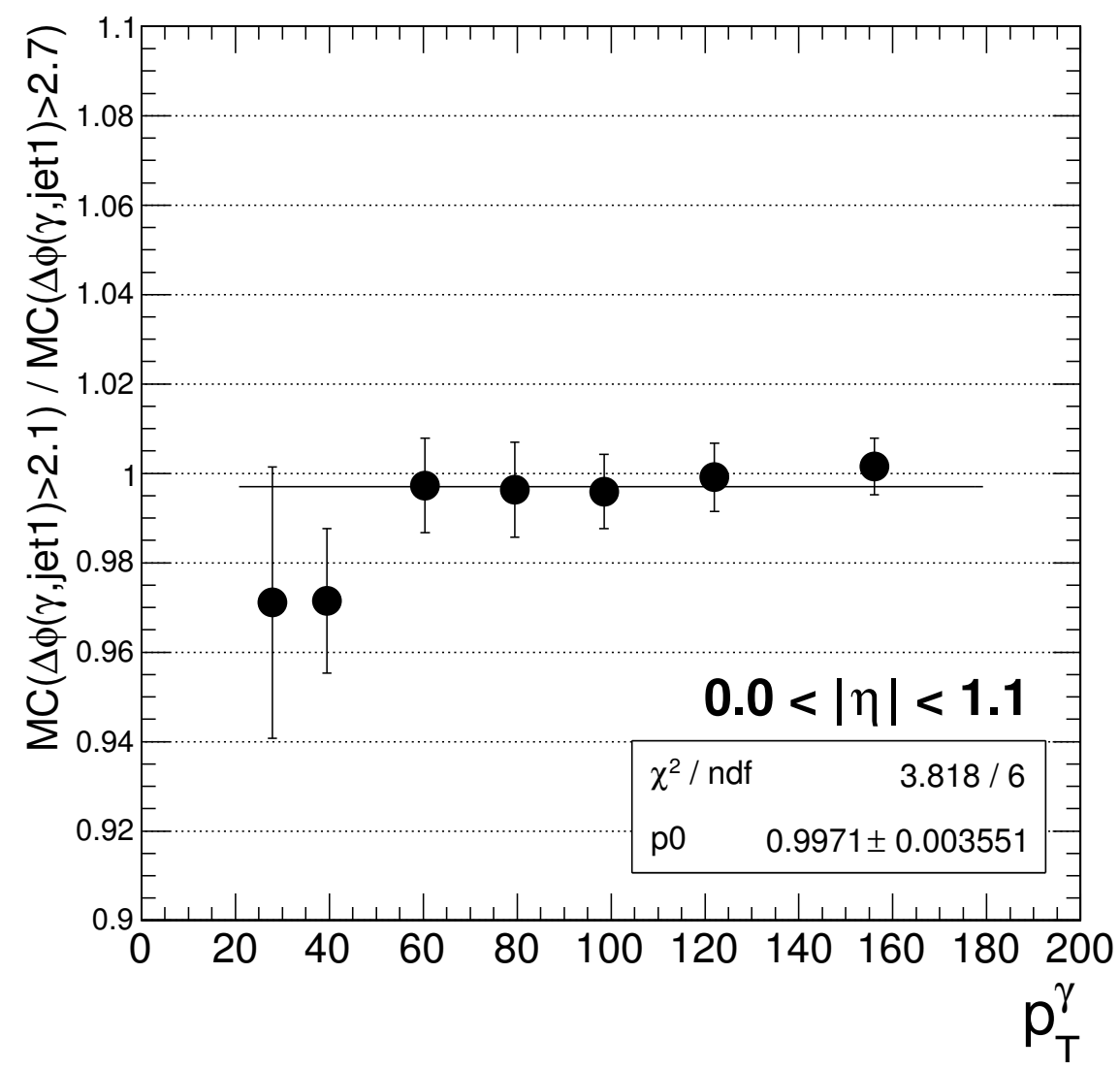

Figure 5.18: Ratio of intrinsic resolutions measured in MC for two different $\Delta \phi$ requirements for $0.0<|\eta|<1.1$ region.

\subsubsection{Uncertainty Due to Imbalance Component}

To calculate intrinsic resolutions, the imbalance measured from the $\mathrm{MC}$ are subtracted both from MC and data, assuming the MC closely describes data. Since various sources can lead to a different imbalance components in data and MC, I evaluated an uncertainty due to the following items:

1. treatment of multi-parton final states, 


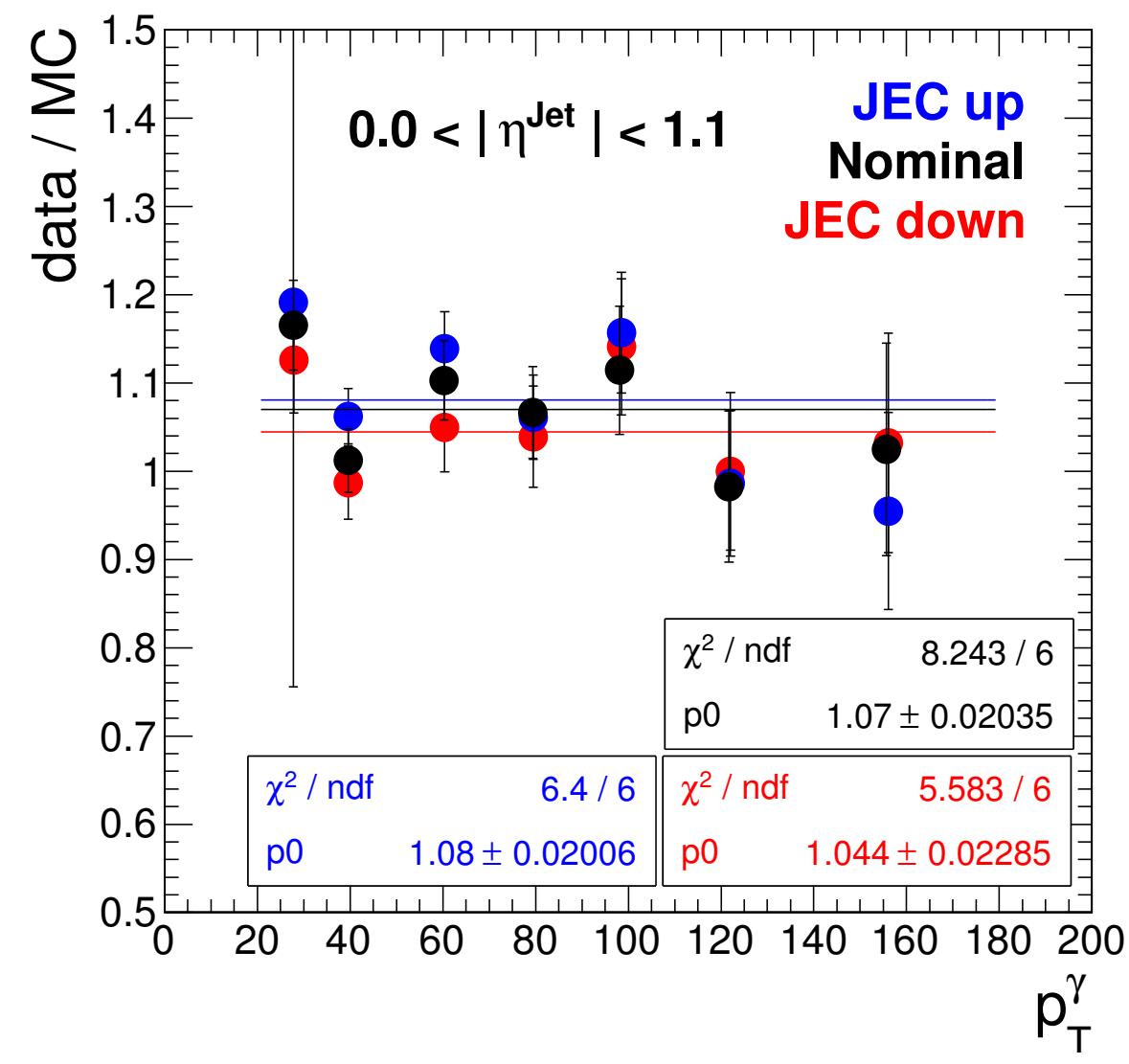

Figure 5.19: Data/MC ratio for nominal (black), JEC up (blue), and JEC down (red) measurements for $0.0<|\eta|<1.1$ region.

2. modeling of hadronization,

3. modeling of $k_{T}$ kick.

Treatment of multi-parton final states: Different event generators deal with parton showering by using different approaches. To understand this, I generated $\gamma+$ jet MC samples with the MADGRAPH [47] and HERWIG [61] event generators and studied the treatment of multi-parton final states by comparing the results with a 
PYTHIA [46] sample. Using these findings, I recalculated the Data/MC ratio for intrinsic resolution and used the relative differences between the measured ratios in the systematic uncertainty calculations. Figure 5.20 shows the measured Data/MC ratios for different MC generators for the first eta bin $(0.0<|\eta|<1.1)$. The nominal ratio (1.070) I calculated with the PYTHIA sample changed to 1.087 for MADGRAPH and 1.068 for HERWIG.

Modeling of hadronization: To study the modeling of hadronization, I recalculated the Data/MC ratios for intrinsic resolutions by turning on and off the PYTHIA hadronization parameter and used the relative difference between the two measured ratios in a systematic uncertainty assignment. The effect of hadronization for imbalance resolutions is shown in Figure 5.21 for $22-32 \mathrm{GeV}$ and $52-72 \mathrm{GeV}$ photon $\mathrm{p}_{\mathrm{T}}$ bins in the fraction of $\mathrm{p}_{\mathrm{T}}^{\mathrm{Jet} 2} / \mathrm{p}_{\mathrm{T}}^{\gamma}$. The measured Data/MC ratios with and without hadronization are shown in Figure 5.22, and the relative difference between the two ratios $(\mid \Delta$ Ratio $\mid)$ is found to be 0.009 .

Modeling of $k_{T}$ kick: A previous CDF study on direct photon production showed that an additional $3-4 \mathrm{GeV} k_{T}$ smearing of initial state partons was needed to match QCD next-to-leading predictions for the observed photon $\mathrm{p}_{\mathrm{T}}$ spectrum from collider data [62]. In PYTHIA, parton showering and initial state radiation (ISR) are already applied. Therefore, a $1 \mathrm{GeV}$ smearing of the second particle jet $\mathrm{p}_{\mathrm{T}}$ is studied to estimate the uncertainty due to mis-modeling of partonic final states, including ISR. Figure 5.23 compares measured imbalance resolutions before and after a $k_{T}$ kick (smearing the second particle jet $\mathrm{p}_{\mathrm{T}}$ ) for $22-32 \mathrm{GeV}$ and $52-72 \mathrm{GeV}$ photon $\mathrm{p}_{\mathrm{T}}$ bins. 
To study the extreme case of a $k_{T}$ kick, a $3 \mathrm{GeV}$ smearing of the second particle jet $\mathrm{p}_{\mathrm{T}}$ is also applied, and the results are compared with nominal measurements. It is seen that the $k_{T}$ kick is more effective on the lower $\mathrm{p}_{\mathrm{T}}$ regions and its effect becomes smaller with increasing photon $\mathrm{p}_{\mathrm{T}}$.

After smearing of the second particle jet $\mathrm{p}_{\mathrm{T}}$ by $1 \mathrm{GeV}$ in the PYTHIA MC,

I recalculated the Data/MC ratio for intrinsic resolutions. The relative differences between the nominal and recalculated Data/MC ratios are used to determine systematies. Figure 5.24 shows the Data/MC ratio for the pseudorapidity range $0.0<|\eta|<$ 1.1 with and without $k_{T}$ smearing. The relative difference between the two measured Data/MC ratios is 0.013 .

The final systematic uncertainties on the Data/MC ratios from the imbalance component is assigned by comparing the results from hadronization, multi-parton final state, and modeling of the $k_{T}$ kick. For each item, the Data/MC ratio is recalculated and the results are compared with the nominal Data/MC ratio. Table 5.4 summarizes how much each item changes the measured Data/MC ratio, and the last column shows the systematic uncertainty assigned to each pseudorapidity range used in this analysis.

\subsubsection{Uncertainty Due to Flavor Composition in QCD and $\gamma+$ jet Sam- ples:}

To estimate the uncertainty of the Data/MC ratios due to jet flavor composition in QCD multijet and $\gamma+$ jet samples, I measured intrinsic resolutions for QCD and $\gamma+$ jet PYTHIA MC samples and calculated their ratio. The intrinsic resolution 
Table 5.4: Relative changes in the measured Data/MC ratios due to mismeasurement of the MC imbalance component and the assigned systematic uncertainties.

\begin{tabular}{|c|c|c|c|c|}
\hline$|\eta|$ & $\begin{array}{c}\text { Multi-parton } \\
\text { final state }\end{array}$ & Hadronization & $\begin{array}{c}\text { Modeling of } \\
k_{T} \text { kick }\end{array}$ & $\begin{array}{c}\text { Assigned } \\
\text { Uncertainty }\end{array}$ \\
\hline $0.0-1.1$ & 0.017 & 0.009 & 0.013 & $\pm 2 \%$ \\
\hline $1.1-1.7$ & 0.03 & 0.005 & 0.014 & $\pm 3 \%$ \\
\hline $1.7-2.3$ & 0.064 & 0.023 & 0.021 & $\pm 4 \%$ \\
\hline $2.3-3.5$ & 0.001 & 0.038 & 0.024 & $\pm 4 \%$ \\
\hline
\end{tabular}

is measured as the width of $p_{T}^{\text {RecJet }} / p_{T}^{\text {ParticleJet }}$ for reconstructed jets with the highest transverse momentum (leading jet) where $\Delta \mathrm{R}=0.1$ matching is used between the leading jet and the particle level jet. $\Delta \mathrm{R}$ is defined as $\sqrt{(\Delta \phi)^{2}+(\Delta \eta)^{2}}$.

Figure 5.25 shows intrinsic resolutions I measured with the QCD and $\gamma+$ jet samples and their ratio with respect to particle level jet $\mathrm{p}_{\mathrm{T}}$. For the $20-300 \mathrm{GeV}$ $\mathrm{p}_{\mathrm{T}}$ region, where the resolutions are measured from the $\gamma+$ jet sample, the intrinsic resolutions differ by $\sim 3 \%$.

To estimate the systematic uncertainty due to flavor composition in QCD and the $\gamma+$ jet sample, I pursued the following assumption. Assuming the measurements from MC are wrong by $30 \%$ (which is a very conservative assumption) the absolute resolution which we assigned a $3 \%$ uncertainty can vary from $2-4 \%$. Since the measurement is done as the Data/MC ratio in the final state, common biases and systematic uncertainties cancel out, and the residual uncertainty is $\sim 1 \%$ [60]. This uncertainty is only applied to the Data/MC ratio when the results from the $\gamma+$ jet 
sample are compared with the dijet sample.

\subsubsection{Uncertainty Due to Pileup}

To study the effect of pileup, the additional large number of soft pp collisions, on the Data/MC measurement I pursued the following approach. I recalculated the Data/MC ratios with pileup-corrected jets and compared the results with the nominal Data/MC ratio where jets are not corrected for pileup. The relative differences between the two measured ratios are assigned as systematic uncertainties.

Since the correction factors derived for the Data/MC ratio are only applied to MC samples produced without the pileup simulation, to calculate the new Data/MC ratios, only the jets in the data are corrected for pileup. The comparison of the measured and intrinsic resolutions for MC, data, and pileup corrected data in the fraction of $\mathrm{p}_{\mathrm{T}}^{\mathrm{Jet} 2} / \mathrm{p}_{\mathrm{T}}^{\gamma}$ for the $22-32,52-72 \mathrm{GeV}$ photon $\mathrm{p}_{\mathrm{T}}$ bins are shown in Figure 5.26 and Figure 5.27. I found that after the pileup correction, the measured resolutions in the data are improved.

A comparison of the Data/MC ratio for intrinsic resolutions with and without pileup-corrected data is shown in Figure 5.28 for $|\eta|<1.1$. The pileup correction to the jets in the data, increases the Data/MC ratio by $\sim 4 \%$ for the barrel region. Table 5.5 summarizes the measured Data/MC ratios with and without the pileup correction and provides systematic uncertainties for the pseudorapidity ranges $(0.0-1.1)$, $(1.1-1.7),(1.7-2.3)$ and $(2.3-3.5)$

However, the data sets used in this analysis are not corrected for pileup. Sys- 
Table 5.5: Data/MC ratio of the measured intrinsic resolutions with (withPUcorr) and without pileup (noPUcorr) correction on data and assigned uncertainties for different jet $\eta$ ranges.

\begin{tabular}{|c|c|c|c|c|}
\hline$|\eta|$ & $\frac{\text { Data (noPUcorr })}{M C(\text { noPUcorr })}$ & $\frac{\text { Data (withPUcorr })}{M C(\text { noPUcorr })}$ & $\left|\Delta\left(\frac{\text { Data }}{M C}\right)\right|$ & Uncertainty \\
\hline $0.0-1.1$ & 1.070 & 1.107 & 0.037 & $\pm 4 \%$ \\
\hline $1.1-1.7$ & 1.102 & 1.123 & 0.021 & $\pm 2 \%$ \\
\hline $1.7-2.3$ & 1.066 & 1.084 & 0.018 & $\pm 2 \%$ \\
\hline $2.3-3.5$ & 1.183 & 1.129 & 0.054 & $\pm 5 \%$ \\
\hline
\end{tabular}

Table 5.6: Data/MC ratio of the measured intrinsic resolutions and assigned uncertainties for different jet $\eta$ ranges.

\begin{tabular}{|c|c|c|c|c|}
\hline$|\eta|$ & Data/MC & $\begin{array}{c}\text { Jet Energy } \\
\text { Correction }\end{array}$ & Extrapolation & $\begin{array}{c}\text { Particle Level } \\
\text { Imbalance }\end{array}$ \\
\hline $0.0-1.1$ & $1.070 \pm 0.020$ & $+0.010 /-0.025$ & $+0.009 /-0.005$ & $+0.020 /-0.020$ \\
\hline $1.1-1.7$ & $1.102 \pm 0.031$ & $+0.004 /-0.024$ & $+0.003 /-0.007$ & $+0.030 /-0.030$ \\
\hline $1.7-2.3$ & $1.066 \pm 0.048$ & $+0.038 /-0.024$ & $+0.010 /-0.005$ & $+0.040 /-0.040$ \\
\hline $2.3-3.5$ & $1.183 \pm 0.062$ & $+0.006 /-0.060$ & $+0.002 /-0.013$ & $+0.040 /-0.040$ \\
\hline
\end{tabular}

tematic uncertainties listed in Table 5.5 are not included in the final systematic uncertainties assigned to Data/MC ratios. This uncertainty should be taken into account only if the resolutions corrected by the Data/MC factor, measured without the pileup subtraction, are applied to the pileup-corrected jets.

Table 5.6 summarizes the Data/MC ratios measured with $\gamma+$ jet events using the $\mathrm{p}_{\mathrm{T}}$ balance technique and their systematic and statistical uncertainties for the pseudorapidity ranges $(0.0-1.1),(1.1-1.7),(1.7-2.3)$ and $(2.3-3.5)$. 
The systematic uncertainties for the Data/MC ratios come from jet energy corrections, extrapolation fit ranges, and particle level imbalance. The contributions from each item are expected to be uncorrelated and the final systematic uncertainties are calculated as the quadrature sum of individual components. The measured Data/MC ratios of intrinsic resolutions with assigned systematic uncertainties are shown in Figure 5.29 for the various jet $\eta$ bins used in this analysis.

\subsection{Results of the Rebalance and Smear Method}

The QCD multijet background is predicted from the multijet data sample with events passing the $\mathrm{H}_{\mathrm{T}}$ triggers described in Section 4.4. The R\&S method is applied using the full (Gaussian core + tails) jet energy resolution functions. The numbers of background events are predicted after applying search selections to the R\&S events.

The predicted events are corrected for the known biases of the method. The first bias comes from the smearing step and is corrected by comparing simulations and $R \& S$ predictions. The relative difference between these two is taken into account as a bias correction and a systematic uncertainty. The second bias comes from the rebalancing procedure and studied by iterating the $R \& S$ method. When we perform the R\&S method on an inclusive multijet sample, the method (first iteration) gives a QCD multijet sample. Reapplying the R\&S method (second iteration) to the produced QCD sample provides a closure test of the rebalancing procedure. The non-closure between the first and second iterations is used as the bias correction. The last bias come from using the momentum of reconstructed soft particles as a good 
Table 5.7: Number of QCD multijet events predicted before and after bias corrections, along with relevant systematic uncertainties [44].

\begin{tabular}{|l|c|c|c|}
\hline & Baseline Sel. & High- $\not_{\mathrm{T}}$ Sel. & High- $\mathrm{H}_{\mathrm{T}}$ Sel. \\
\hline Nominal Prediction & 39.4 & 0.18 & 19.0 \\
Bias-corrected Prediction & $29.7 \pm 15.2$ & $0.16 \pm 0.10$ & $16.0 \pm 7.9$ \\
\hline
\end{tabular}

estimator of true momentum of detector-level soft particles and is corrected by using the difference between the first and second iterations of the R\&S method.

The predicted numbers of events before and after bias corrections for different search regions are listed in Table 5.7. The predicted numbers include the systematic uncertainties coming from the bias corrections, jet momentum resolution, the effect of pileup, event selections, trigger selection on the control samples, and seed sample statistics. 


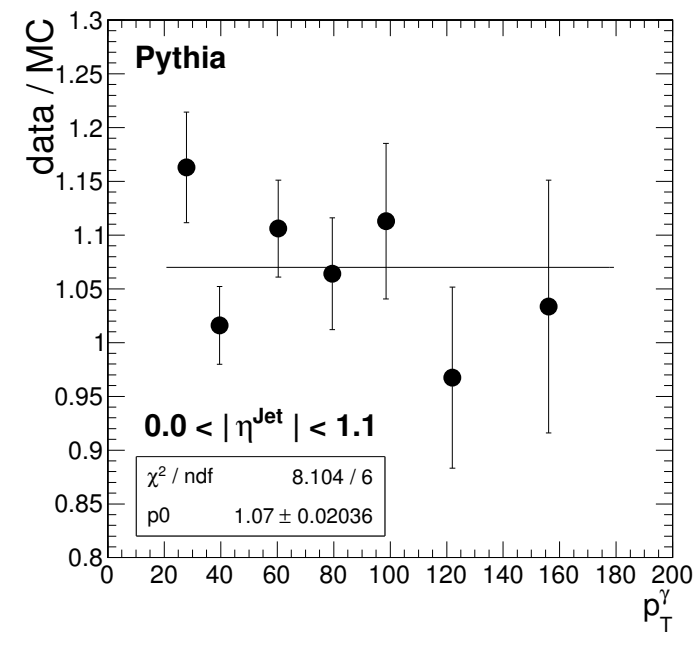

(a) PYTHIA

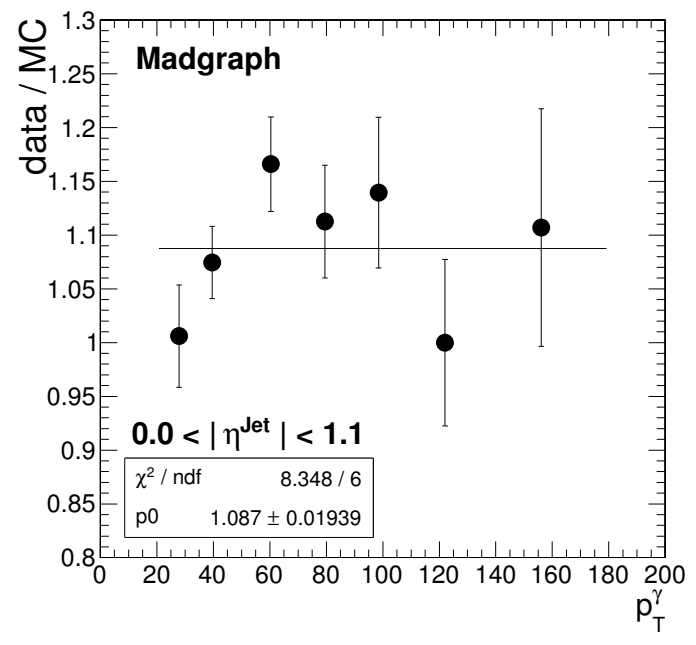

(b) MADGRAPH

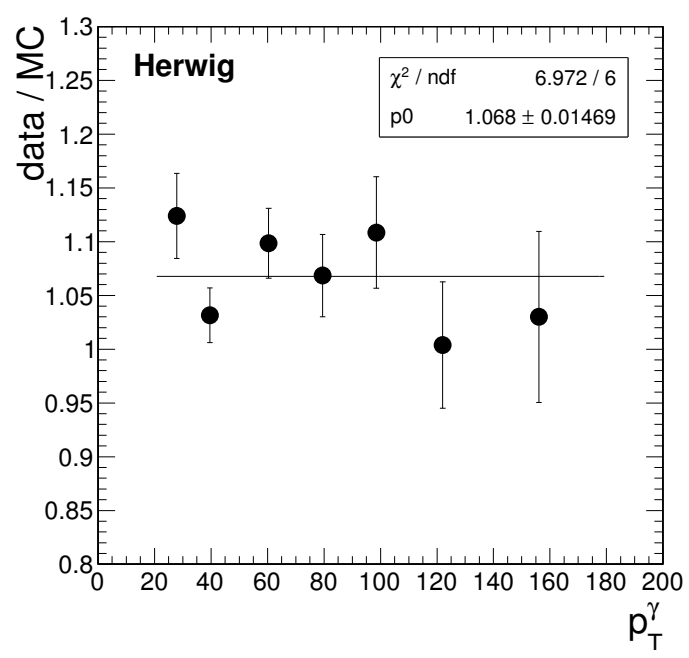

(c) HERWIG

Figure 5.20: Data/MC ratios for intrinsic resolution for different MC generators; PYTHIA (a), MADGRAPH (b), and HERWIG (c). The distributions are shown for pseudorapidity range $0.0<|\eta|<1.1$. 


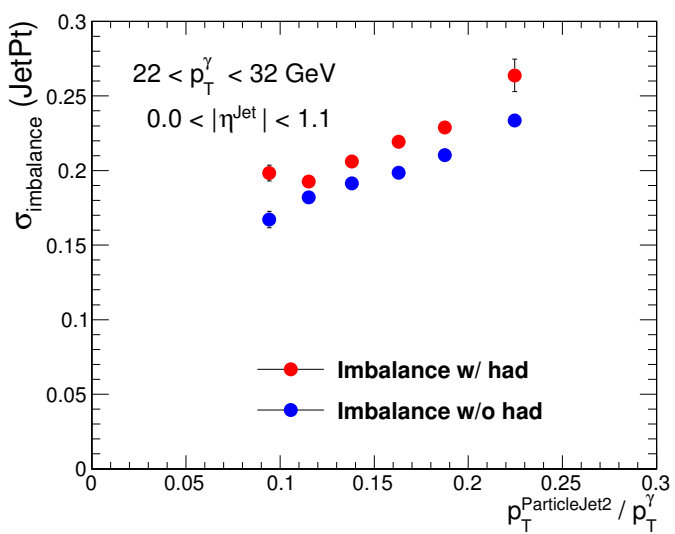

(a) $22 \mathrm{GeV}<p_{T}^{\gamma}<32 \mathrm{GeV}$

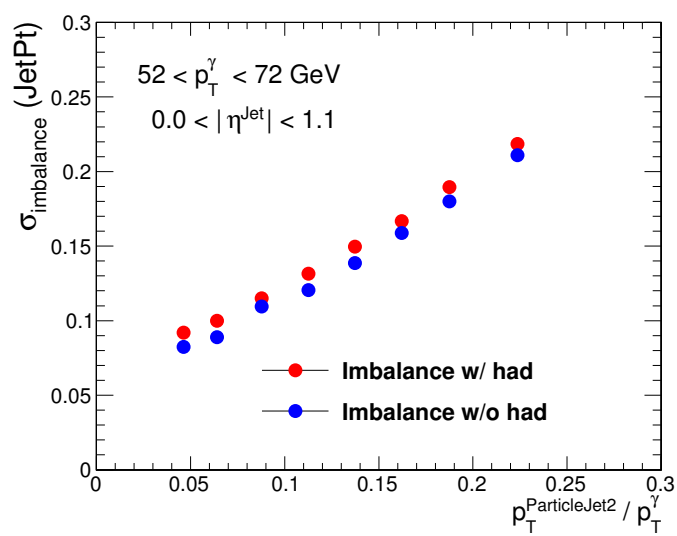

(b) $52 \mathrm{GeV}<p_{T}^{\gamma}<72 \mathrm{GeV}$

Figure 5.21: Measured imbalance resolutions in the fraction of $\mathrm{p}_{\mathrm{T}}^{\mathrm{Jet} 2} / \mathrm{p}_{\mathrm{T}}^{\gamma}$ for $22-32$, 52-72 GeV photon $\mathrm{p}_{\mathrm{T}}$ bins. The distributions are shown for pseudorapidity range $0.0<|\eta|<1.1$. 


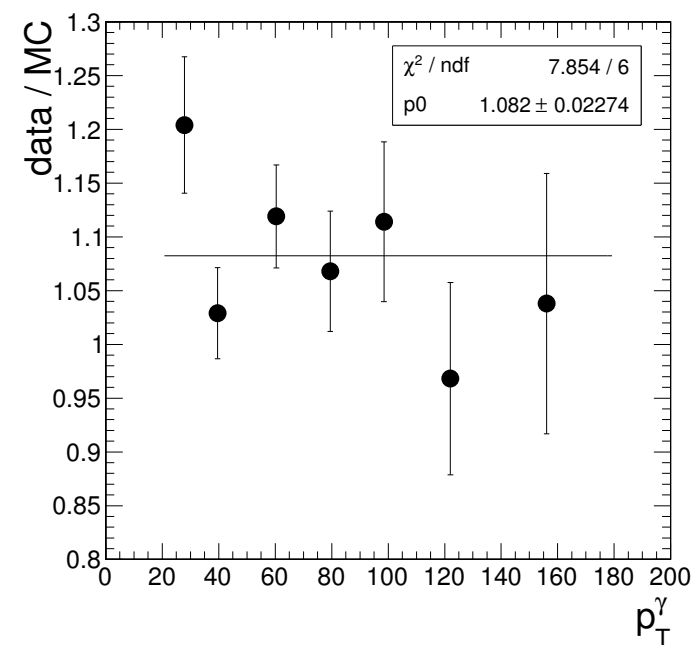

(a) With hadronization

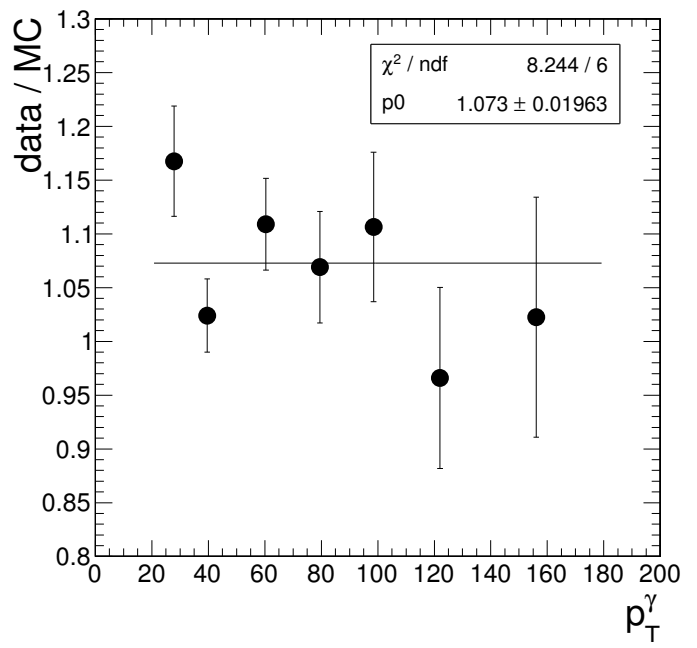

(b) Without hadronization

Figure 5.22: Data/MC ratio for intrinsic resolutions measured from PYTHIA with and without hadronization. The distributions are shown for pseudorapidity range $0.0<|\eta|<1.1$. 


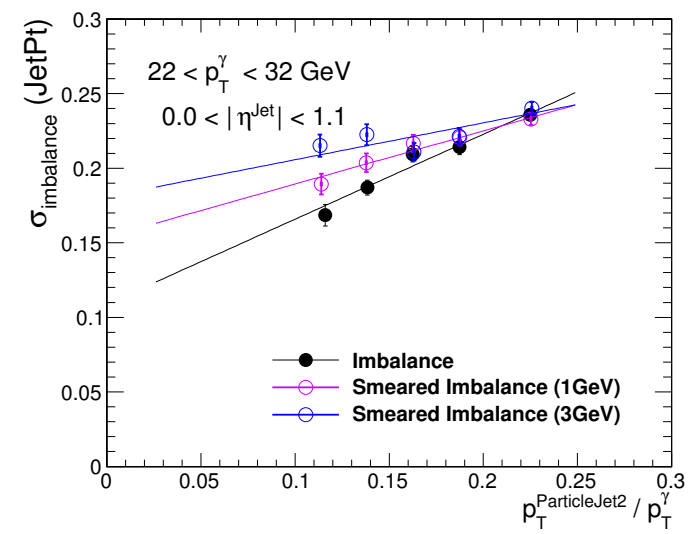

(a) $22 \mathrm{GeV}<p_{T}^{\gamma}<32 \mathrm{GeV}$

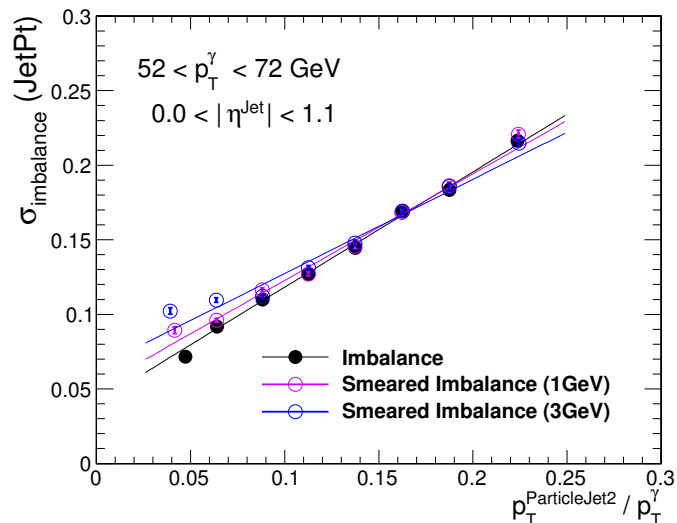

(b) $52 \mathrm{GeV}<p_{T}^{\gamma}<72 \mathrm{GeV}$

Figure 5.23: Measured imbalance resolutions in the fraction of $\mathrm{p}_{\mathrm{T}}^{\mathrm{Jet} 2} / \mathrm{p}_{\mathrm{T}}^{\gamma}$ for $22-32$, 52-72 GeV photon $\mathrm{p}_{\mathrm{T}}$ bins. The distributions are shown for pseudorapidity range $0.0<|\eta|<1.1$. The nominal distributions are shown by black markers, whereas smeared imbalance distributions are shown by violet (1 GeV smearing) and blue (3 GeV smearing) markers. 


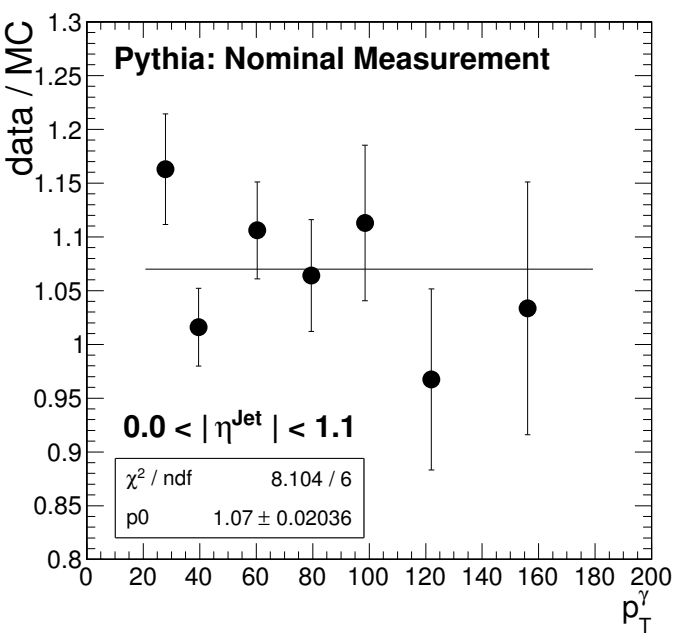

(a) Nominal Measurement

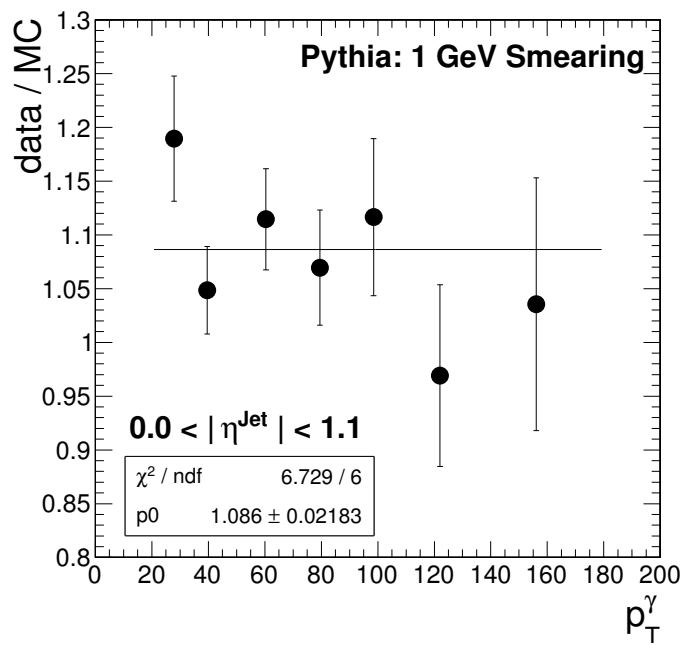

(b) $1 \mathrm{GeV} k_{T}$ smearing

Figure 5.24: Data/MC ratio for intrinsic resolutions measured by using PYTHIA with (right) and without (left) $k_{T}$ smearing. The distributions are shown for pseudorapidity range $0.0<|\eta|<1.1$. 


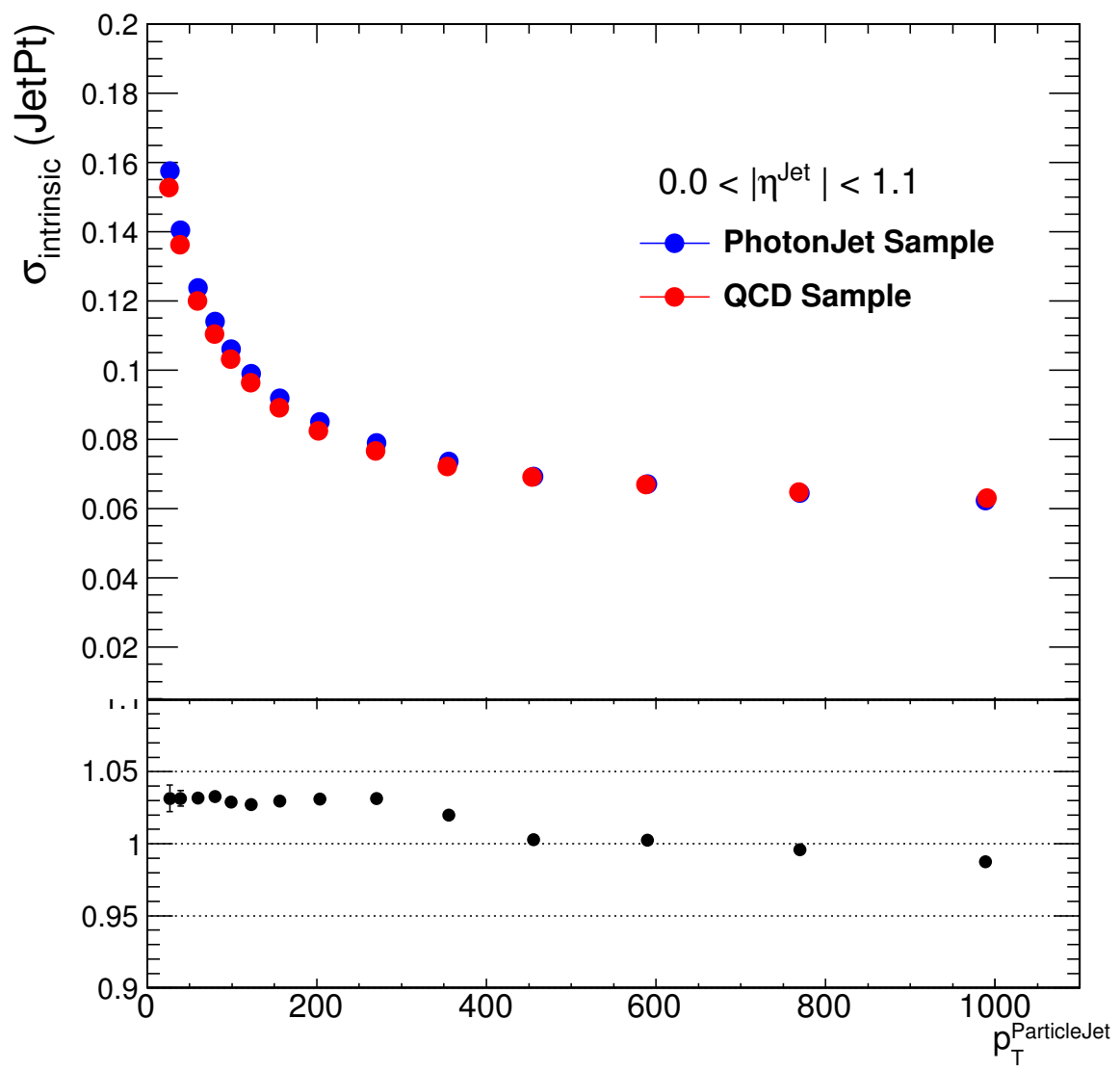

Figure 5.25: Intrinsic resolutions (top) for QCD and $\gamma+$ jet samples with respect to particle level jet $\mathrm{p}_{\mathrm{T}}$ for the $0.0<|\eta|<1.1$ region and the ratio of $\sigma_{\text {photon }} / \sigma_{\mathrm{QCD}}$ (bottom). 

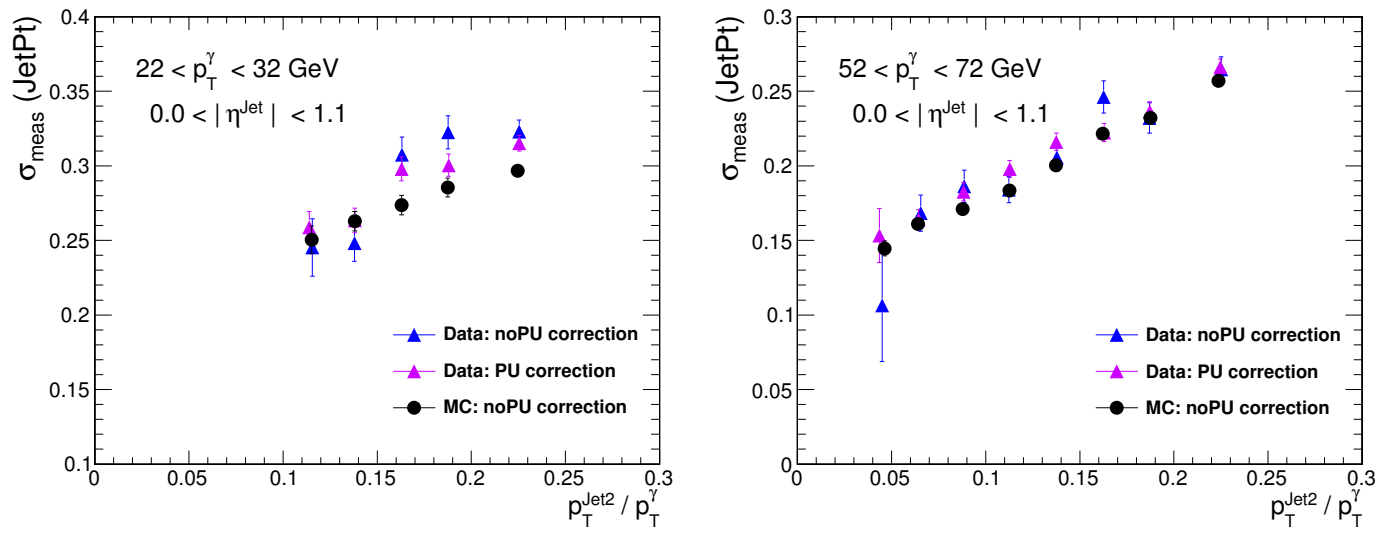

Figure 5.26: Measured resolutions, $\sigma\left(p_{T}^{\text {Jet }} / p_{T}^{\gamma}\right)$, in the fraction of $\mathrm{p}_{\mathrm{T}}^{\mathrm{Jet} 2} / \mathrm{p}_{\mathrm{T}}^{\gamma}$ for $22-32$ $\mathrm{GeV}$ (left), 52-72 GeV (right) photon $\mathrm{p}_{\mathrm{T}}$ bins for the pseudorapidity range $|\eta|<1.1$.
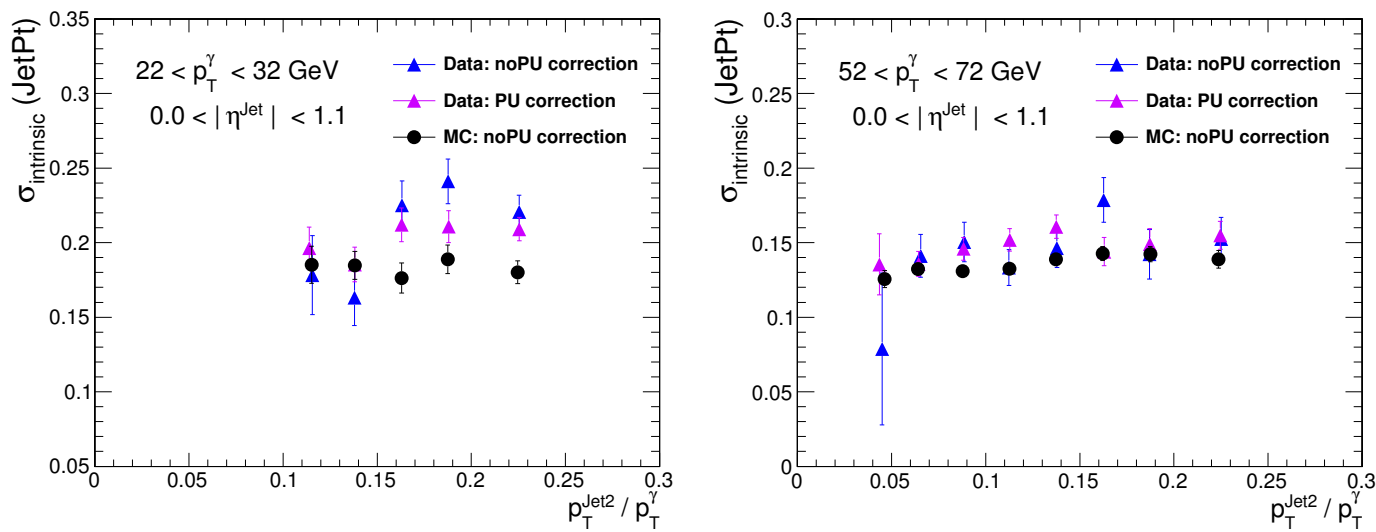

Figure 5.27: Intrinsic resolutions in the fraction of $\mathrm{p}_{\mathrm{T}}^{\mathrm{Jet} 2} / \mathrm{p}_{\mathrm{T}}^{\gamma}$ for $22-32 \mathrm{GeV}$ (left), 52-72 GeV (right) photon $\mathrm{p}_{\mathrm{T}}$ bins for the pseudorapidity range $|\eta|<1.1$. 

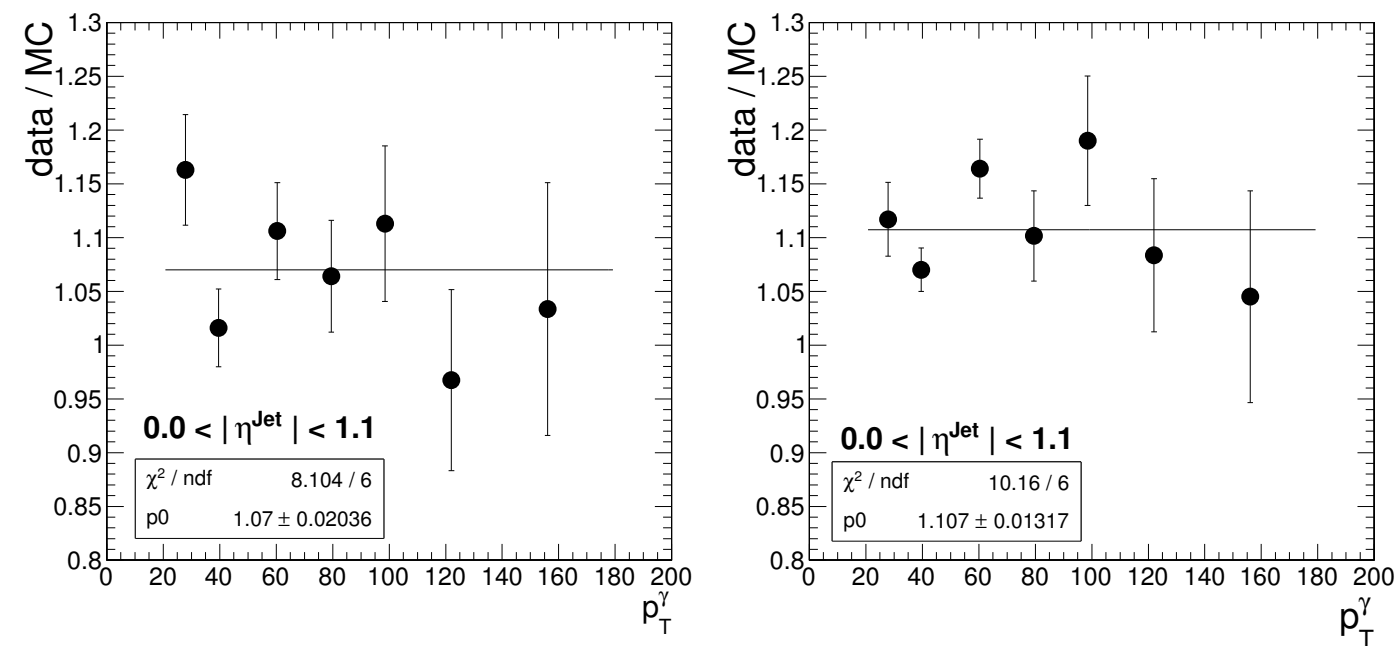

Figure 5.28: Data/MC ratio for without (left) and with (right) pileup correction in data for $|\eta|<1.1$. 


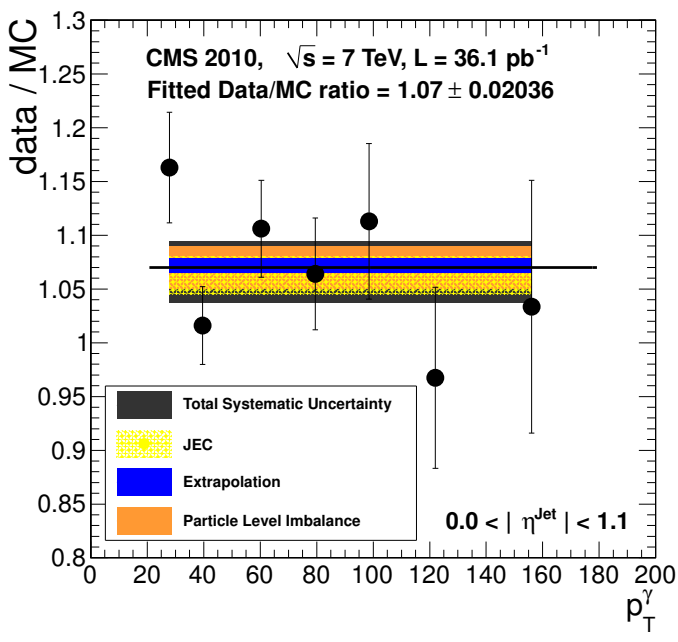

(a) $0.0<\left|\eta^{\text {Jet }}\right|<1.1$

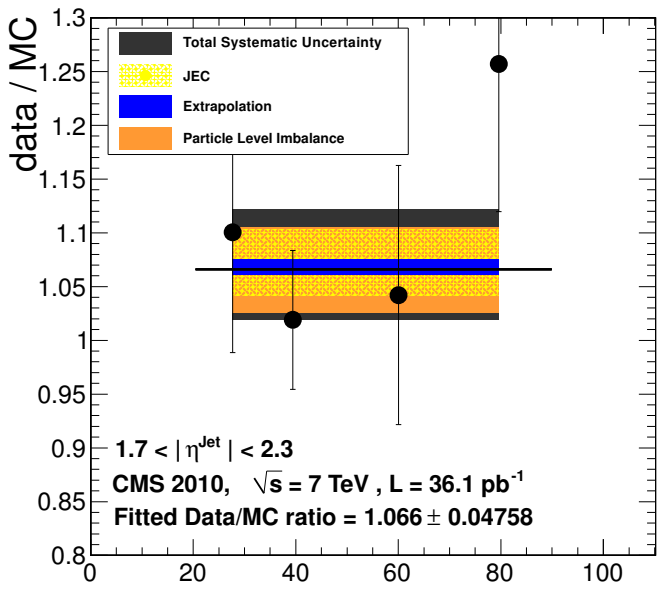

(c) $1.7<\left|\eta^{\mathrm{Jet}}\right|<2.3$

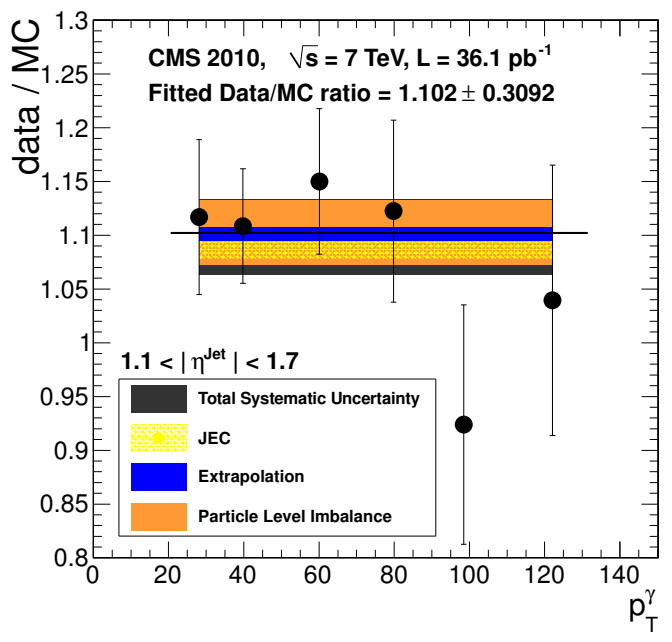

(b) $1.1<\left|\eta^{\text {Jet }}\right|<1.7$

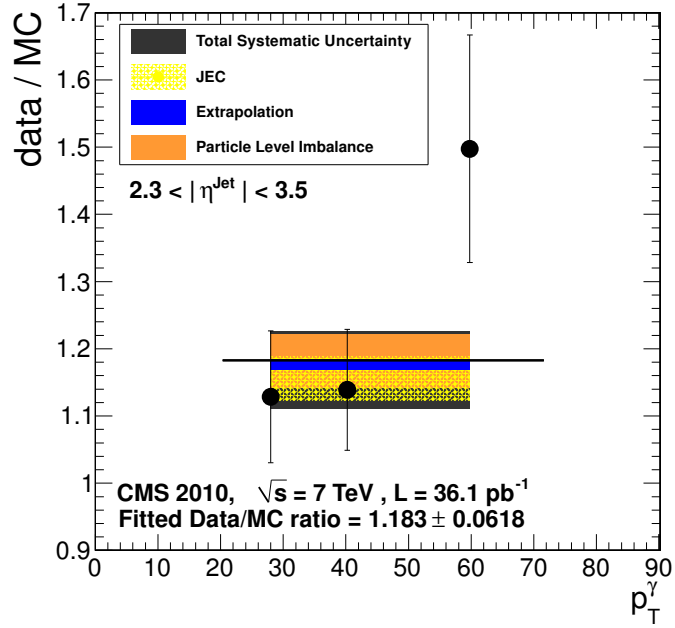

(d) $2.3<\left|\eta^{\text {Jet }}\right|<3.5$

Figure 5.29: Extrapolated ratio of the intrinsic resolutions measured in data and Monte Carlo for various bins of jet $\eta$. 


\section{CHAPTER 6 \\ RESULTS OF INCLUSIVE SEARCH FOR NEW PHYSICS}

\subsection{Results and Limits}

The number of events we observed in data and the number of predicted background events are summarized in Table 6.1 for the baseline, High- $\breve{H}_{\mathrm{T}}$, and High- $\mathrm{H}_{\mathrm{T}}$ selections. We did not observe an excess in either High- $\not_{\mathrm{T}}$ or High- $\mathrm{H}_{\mathrm{T}}$ search regions.

Table 6.1: Predicted number of events from different background components.

\begin{tabular}{|l|c|c|c|}
\hline Background & Baseline Sel. & High- $\mathrm{H}_{\mathrm{T}}$ Sel. & High-H $\mathrm{H}_{\mathrm{T}}$ Sel. \\
\hline $\mathrm{Z}(\nu \bar{\nu})+$ jets & $26.3 \pm 4.8$ & $7.11 \pm 2.2$ & $8.4 \pm 2.3$ \\
$\mathrm{~W} / t \bar{t} \rightarrow \mathrm{e}, \mu, \mathrm{X}$ & $33.0 \pm 8.1$ & $4.8 \pm 1.9$ & $10.9 \pm 3.4$ \\
$\mathrm{~W} / t \bar{t} \rightarrow \tau_{h}, \mathrm{X}$ & $22.3 \pm 4.6$ & $6.7 \pm 2.1$ & $8.5 \pm 2.5$ \\
QCD multijet & $29.7 \pm 15.2$ & $0.16 \pm 0.10$ & $16.0 \pm 7.9$ \\
\hline Total background & $111.3 \pm 18.5$ & $18.8 \pm 3.5$ & $43.8 \pm 9.2$ \\
\hline Nb. events in data & 111 & 15 & 40 \\
\hline 95\% CL upper limit on signal & 40.4 & 9.6 & 19.6 \\
\hline
\end{tabular}

The given systematics include both systematic and statistical uncertainties.

The $95 \%$ confidence level (CL) upper limit on the number of possible signal events is given on the last line [44].

We obtained the expected number of signal events using a simulated LM1 sample together with the related experimental and theoretical systematic uncertainties. Most of those uncertainties are model-dependent since they depend on the event kinematics. The systematic uncertainties of the signals are as follows: 
Experimental Uncertainties: With $8 \%$ value, the largest contribution on the LM1 benchmark point comes from the jet energy scale and resolution measurements. We determined the uncertainties due to lepton veto and trigger selections to be $2 \%$ and $1 \%$, respectively, and the inefficiency of event rejection due to masked ECAL channels [63] on LM1 is $1.5 \%$. We determined that the other experimental uncertainties such as the event cleaning procedure and the presence of pileup interactions corresponding to the 2010 data taking conditions are negligible.

Theoretical Uncertainties: All theoretical uncertainties are considered to be model dependent. The factorization and renormalization scale uncertainties in the NLO cross section corrections are $16 \%$ and they are the largest uncertainties on the LM1 benchmark point. The uncertainties in the parton distribution functions and initial state radiation are $3 \%$ and $2 \%$. We found that, the final state radiation uncertainty is negligible.

Luminosity and Statistical Uncertainties: The luminosity uncertainty is assigned is $4 \%$ [64], and we determined a $2 \%$ statistical uncertainty on the LM1 benchmark point.

The probability distributions corresponding to each uncertainty source are convolved to obtain the probability distributions for each and the overall background estimation. The resulting distribution is fitted by a Gaussian function, and the mean and RMS values are used as the central value and uncertainty in the limit calculations [44]. 

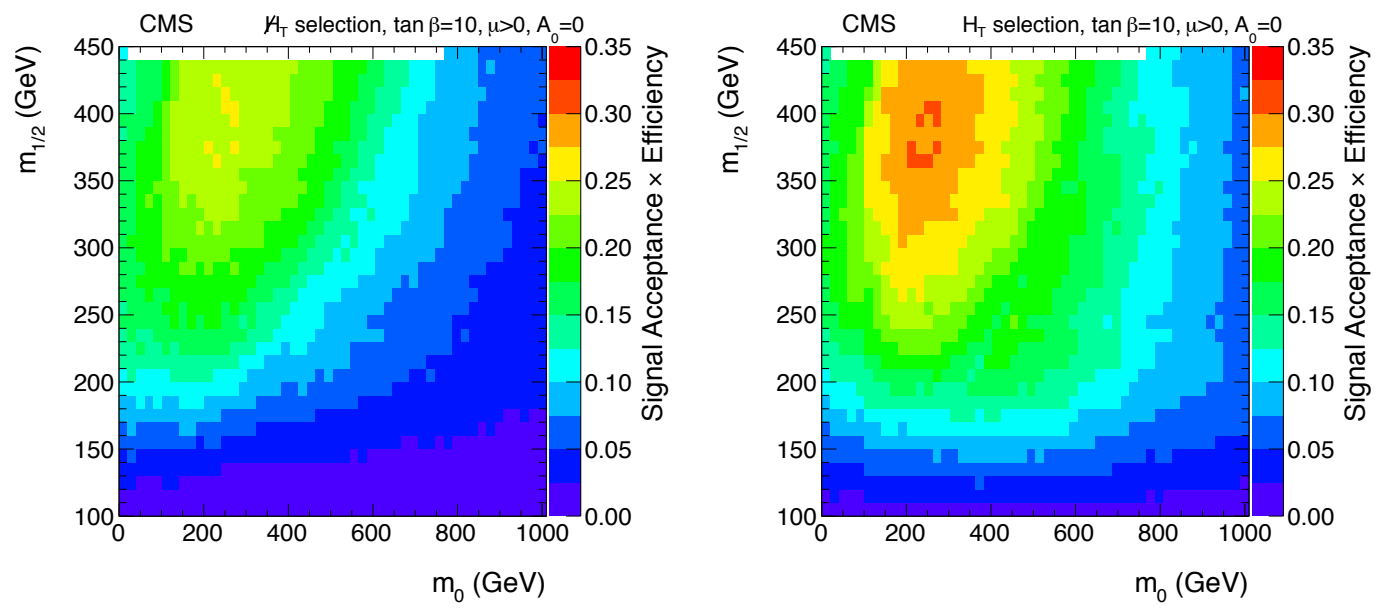

Figure 6.1: Signal efficiency for High- $\mathrm{H}_{\mathrm{T}}$ (left) and High- $\mathrm{H}_{\mathrm{T}}$ (right) selections as a function of $m_{0}$ and $m_{1 / 2}$ for the CMSSM phase space with $\tan \beta=10, \mu>0$, and $A_{0}=0[44]$.

\subsection{Interpretation within the CMSSM}

We processed our results in the $m_{0}$ and $m_{1 / 2}$ CMSSM plane with $\tan \beta=10$ and varied the parameters $m_{0}$ and $m_{1 / 2}$ in $10 \mathrm{GeV}$ steps. We used the leading order ISAJET [65] signal cross sections corrected by next-to-leading-order $\mathrm{K}$ factors that are calculated by PROSPINO [51]. The total signal efficiencies in the CMSSM $m_{0}$

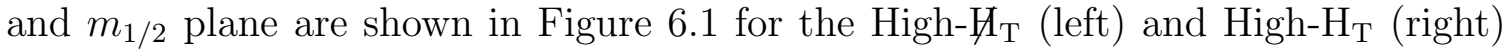
selections. The variation for the High- $\bigsqcup_{\mathrm{T}}$ selection is between $20-30 \%$, whereas it is between $10-20 \%$ for the High- $\mathrm{H}_{\mathrm{T}}$ selection.

For the expected upper limit calculation on the CMSSM cross sections we used the modified frequentist procedure $[66,67]$ with a likelihood ratio test-statistic. The 
limits on the cross sections are obtained using the data-driven background methods under no signal hypothesis [44]. The 95\% CL upper limits for CMSSM are shown in Figures 6.2 and 6.3 in $m_{0}-m_{1 / 2}$ and gluino-squark (bottom) mass planes for $\tan \beta=10, \mu>0$, and $A_{0}=0$. The contours shown in Figures 6.2 and 6.3 are drawn with respect to the best sensitivity of High- $\boldsymbol{h}_{\mathrm{T}}$ and High- $\mathrm{H}_{\mathrm{T}}$ search selections. The lower $m_{0}$ values are dominated by High- $h_{\mathrm{T}}$ selection whereas for the higher $m_{0}\left(m_{0}>\right.$ $450 \mathrm{GeV}$ ) values the High- $\mathrm{H}_{\mathrm{T}}$ selection has more effect. In Figure 6.2, a limit from the previously published CMS supersymmetry search in hadronic events [68] based on the event shape observable $\alpha_{T}$ [69] is also shown. Since we have a better signal selection efficiency, our analysis is able to improve on the limits set by the $\alpha_{T}$ analysis.

\subsection{Interpretation with Simplified Models}

It is also possible to study new physics with a more general framework by using simplified model spectra (SMS) [35-37]. The idea of introducing the simplified model spectra is to remove the complexity of the models with several parameters that describe the data. Simplified models define a small parameter space which allows us to compare experimental results with any complete model.

For the simplified models we used in this study, the assumption is the new particles are produced in pairs and their decay products are stable and weakly interacting massive particles (LSP). The particles produced in these interactions are identified as the partners of quarks and gluons (i.e. squarks and gluinos for supersymmetry). Two benchmark simplified models (Fig. 6.4) are studied for the jets $+h_{\mathrm{T}}$ signature. 


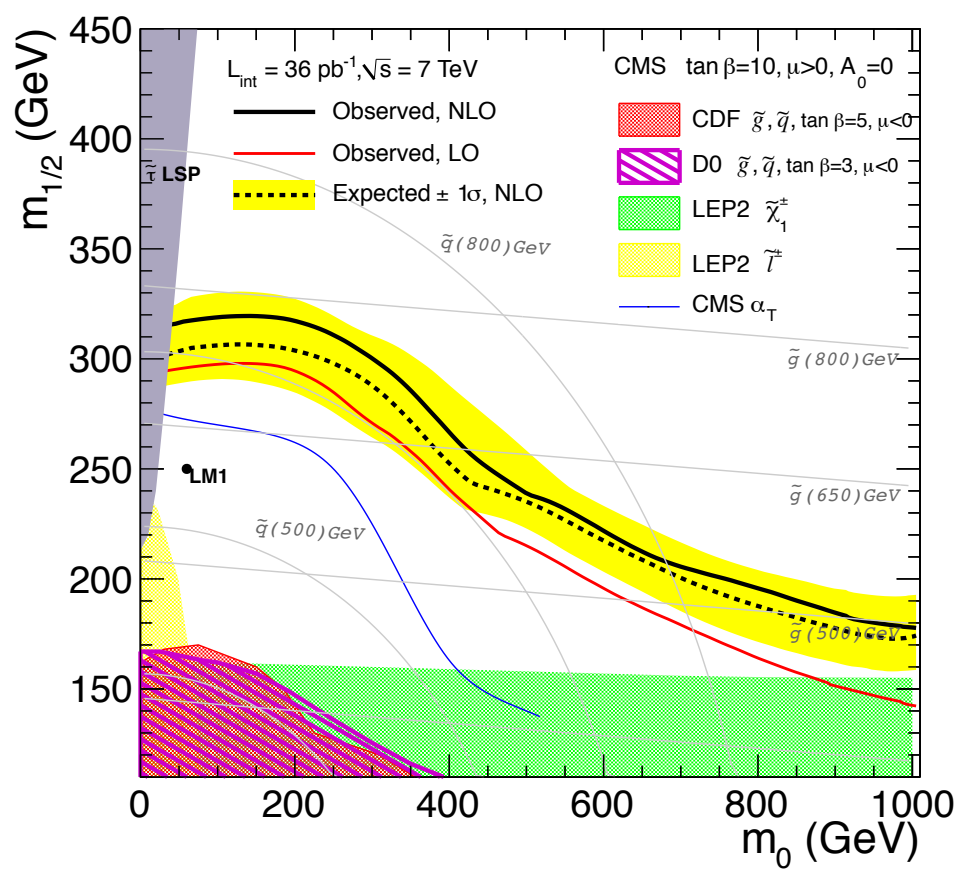

Figure 6.2: Measured (solid black line) and expected (dashed black line with $\pm 1 \sigma$ band) $95 \%$ CL exclusion contour at NLO in the CMSSM $m_{0}-m_{1 / 2}$ mass planes. The measured LO exclusion contour (solid red line) is also shown. The area below the curves is excluded [44]. 


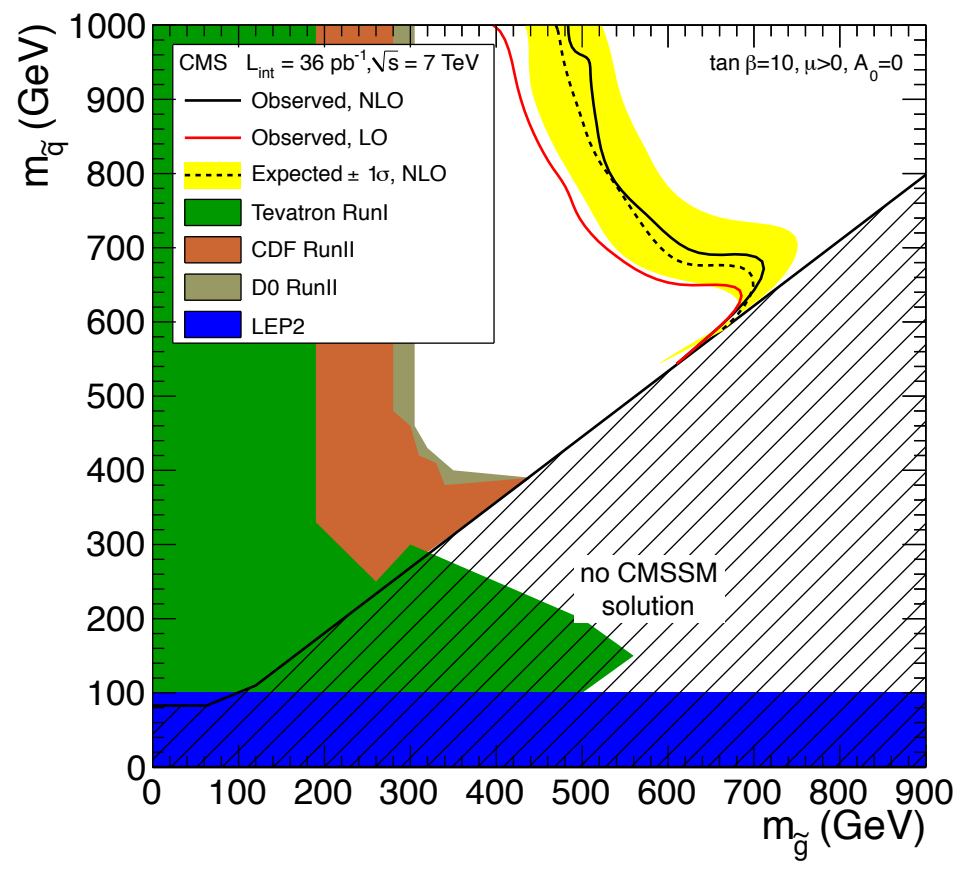

Figure 6.3: The 95\% CL upper limits in the CMSSM gluino-squark mass planes for LO and NLO cross sections [44]. 
These are

- pair produced gluinos, where each gluino decays to two light quarks and the LSP.

- pair produced squarks, where each squark decays to one jet and the LSP.

The simplified models are simulated with PYTHIA and the CTEQ6L1 parton distribution functions [48], and parametrized with the CMS detector simulation. The samples are generated for a range of masses of the particles involved, which provides more mass splitting compared to CMSSM [44].
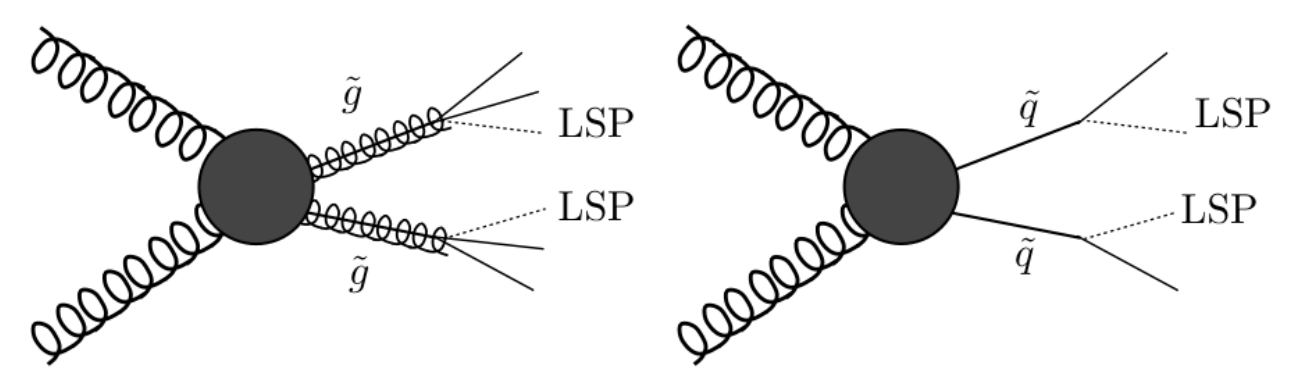

Figure 6.4: Simplified model diagrams. Left: gluino pair production; right: squark pair production.

In Figure $6.5(6.6)$, the total signal efficiencies for the high- $H_{\mathrm{T}}$ and high$\mathrm{H}_{\mathrm{T}}$ selections are shown for the simplified model spectra for gluino (squark) pair production. The efficiencies are shown as a function of gluino (squark) and the LSP masses. Only the lower half of the planes is filled because the model is only valid 
when the gluino (squark) mass is larger then the LSP mass. The signal acceptance increases with a higher mass splitting. The acceptance is low on the diagonal where mass splitting between the decayed particle and LSP is small and the jets are produced with low transverse momentum.
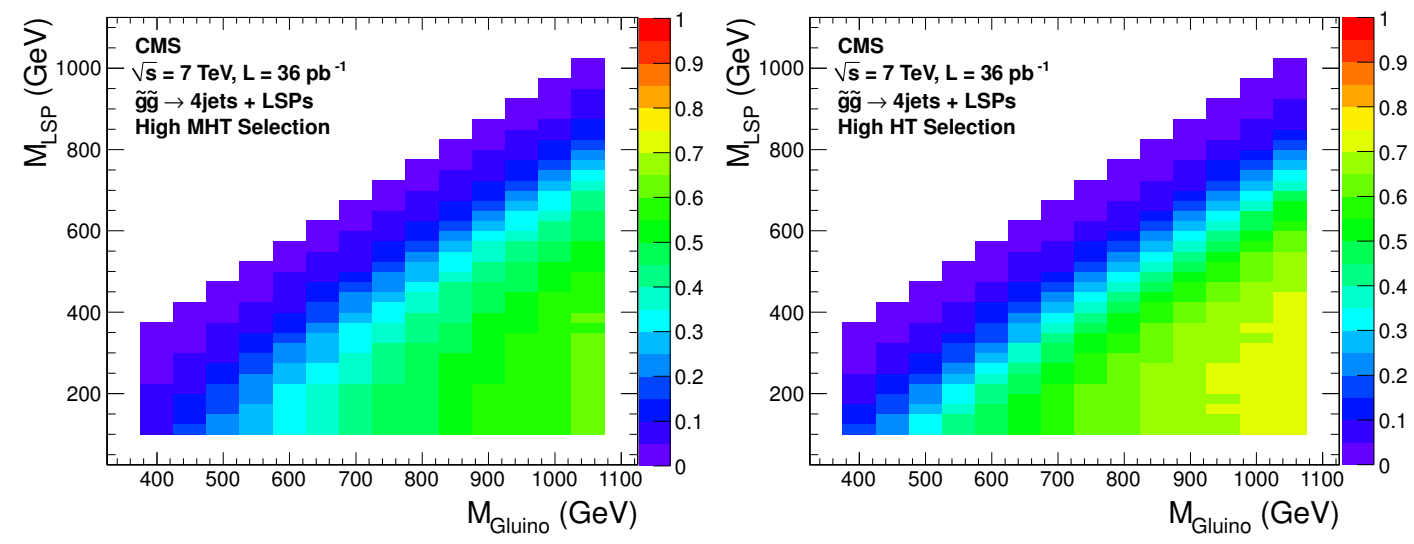

Figure 6.5: High- $\mathrm{H}_{\mathrm{T}}$ (left) and High- $\mathrm{H}_{\mathrm{T}}$ (right) selection efficiencies for gluino production as a function of gluino and LSP mass.

A Bayesian framework with a flat prior for the signal [22] is used in the limit calculation of simplified model spectra. The jet energy scale and resolution, isolated lepton veto, the inefficiency of event rejection by using masked ECAL channels, the trigger efficiency, the initial and final state radiations, the parton distribution functions, the luminosity, and the statistical uncertainties are incorporated as signal acceptance and signal efficiency uncertainties.

The experimental uncertainties for the gluino and squark pair productions are 

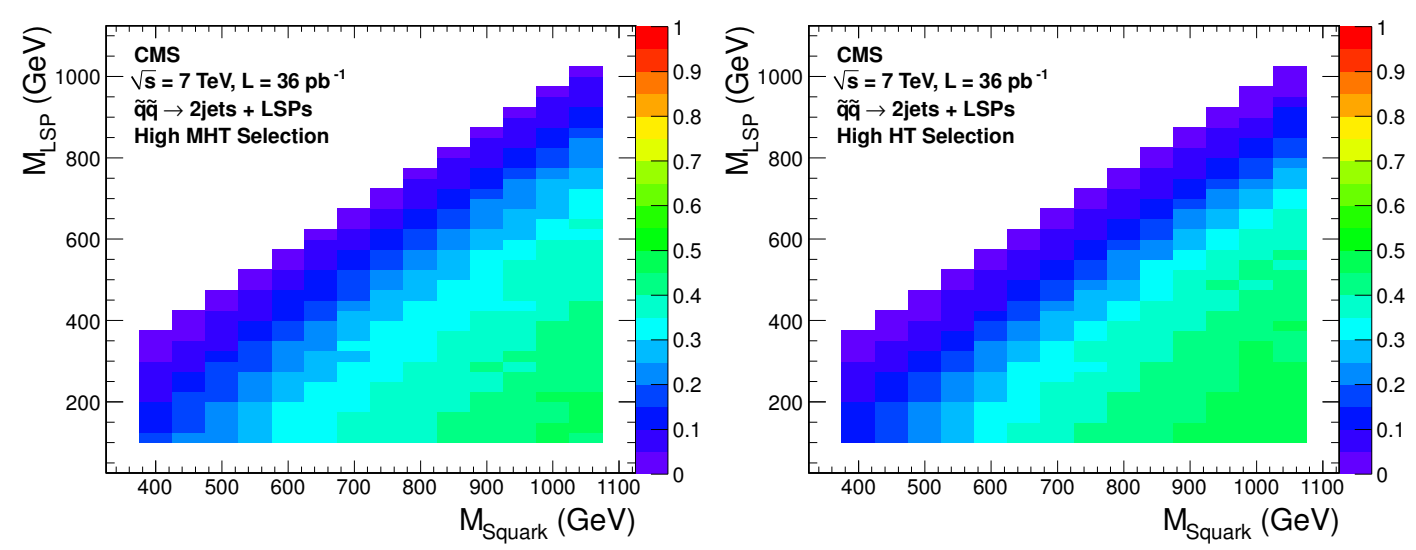

Figure 6.6: High- $\mathrm{H}_{\mathrm{T}}$ (left) and High- $\mathrm{H}_{\mathrm{T}}$ (right) selection efficiencies for squark production as a function of squark and LSP mass.

shown in Figures 6.7 and 6.8 for the High- $\bigsqcup_{\mathrm{T}}$ (left) and High- $\mathrm{H}_{\mathrm{T}}$ (right) selections, whereas the theoretical uncertainties are shown in Figures 6.9 and 6.10.

The 95\% CL upper limits on $\sigma \times \mathrm{Br}$ for gluino and squark pair productions are shown in Figures 6.11 and 6.12 for the High- $\mathrm{H}_{\mathrm{T}}$ (left) and High- $\mathrm{H}_{\mathrm{T}}$ (right) search selections. These upper limits on the cross sections can be translated into a limit on any physics theory such as SUSY or Unified Extra Dimensions (UED). We found that, due to lower signal acceptance and higher uncertainty, excluded cross sections are weaker on the diagonal where mass splitting is small.

We then compared the measured upper limits on the cross sections to the reference NLO cross sections from PROSPINO [51]. These cross sections are used to translate upper limits on the cross sections to limits on new particle masses [44]. The contours given in Figures 6.11 and 6.12 show the points where limit on $(\sigma \times \mathrm{Br})=\sigma_{\text {ref }}$ 

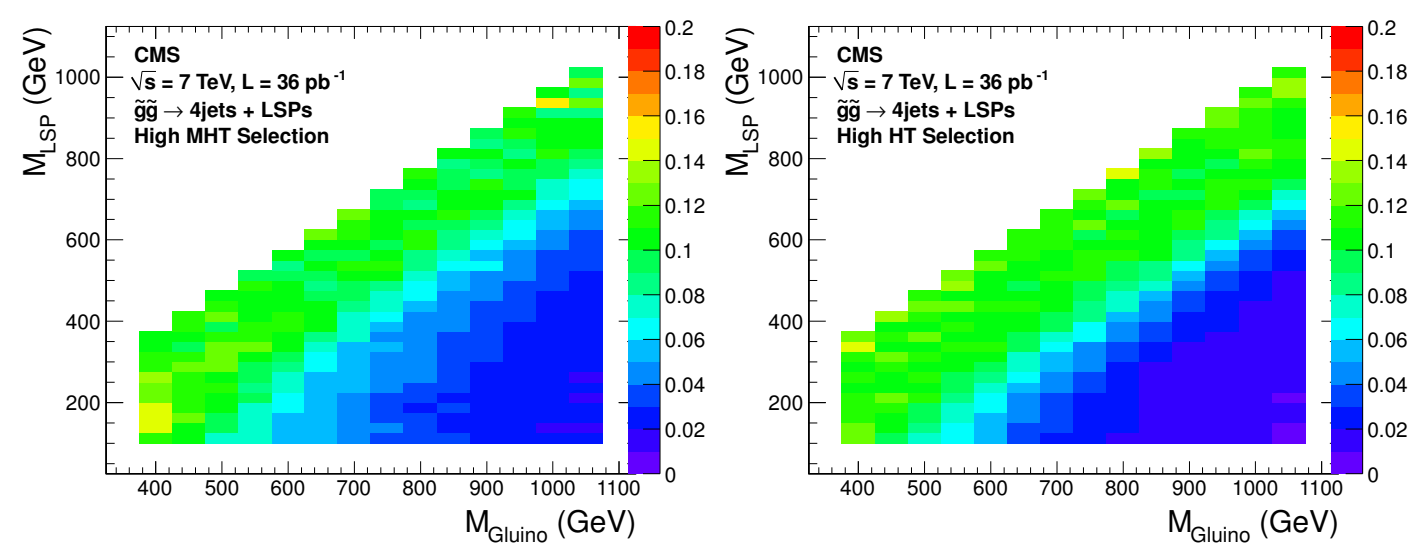

Figure 6.7: Experimental uncertainty on the High- $\mathrm{H}_{\mathrm{T}}$ (left) and High- $\mathrm{H}_{\mathrm{T}}$ (right) selection efficiency for gluino production as a function of the gluino and LSP mass.
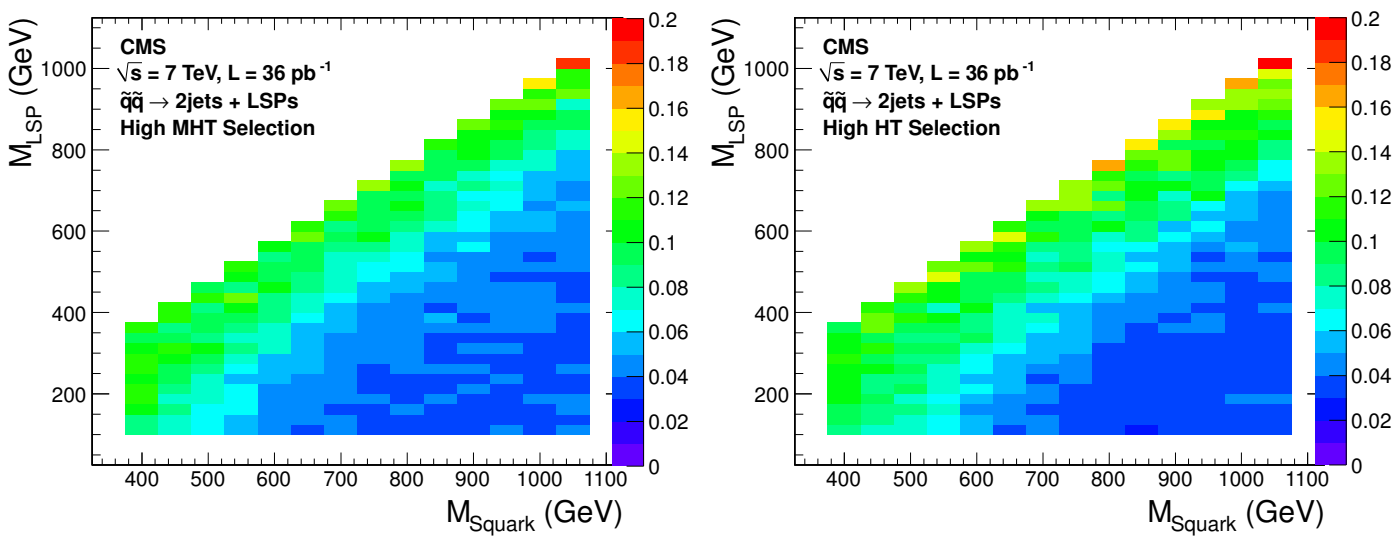

Figure 6.8: Experimental uncertainty on the High- $H_{\mathrm{T}}$ (left) and High- $\mathrm{H}_{\mathrm{T}}$ (right) selection efficiency for squark production as a function of squark and LSP mass. 

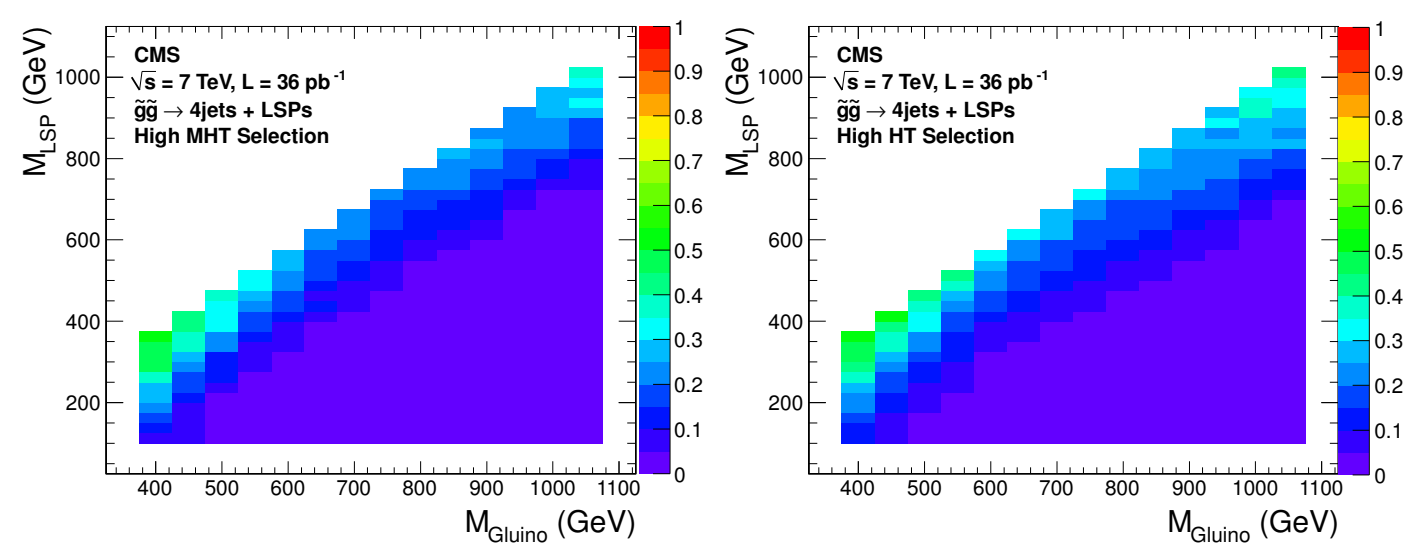

Figure 6.9: Theoretical uncertainty on the High- $\mathrm{H}_{\mathrm{T}}$ (left) and High- $\mathrm{H}_{\mathrm{T}}$ (right) selection efficiency for gluino production as a function of the gluino and LSP mass.
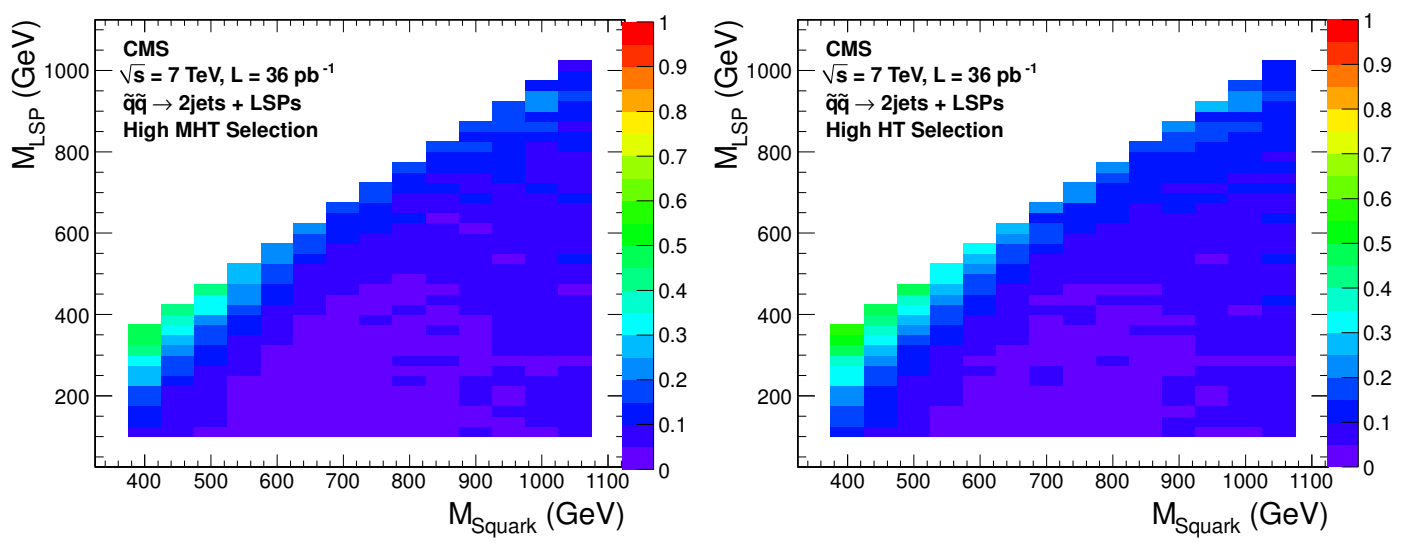

Figure 6.10: Theoretical uncertainty on the High- $\bigsqcup_{\mathrm{T}}$ (left) and $\mathrm{High}-\mathrm{H}_{\mathrm{T}}$ (right) selection efficiency for squark production as a function of squark and LSP mass. 
and three times this cross section (which can be excluded).
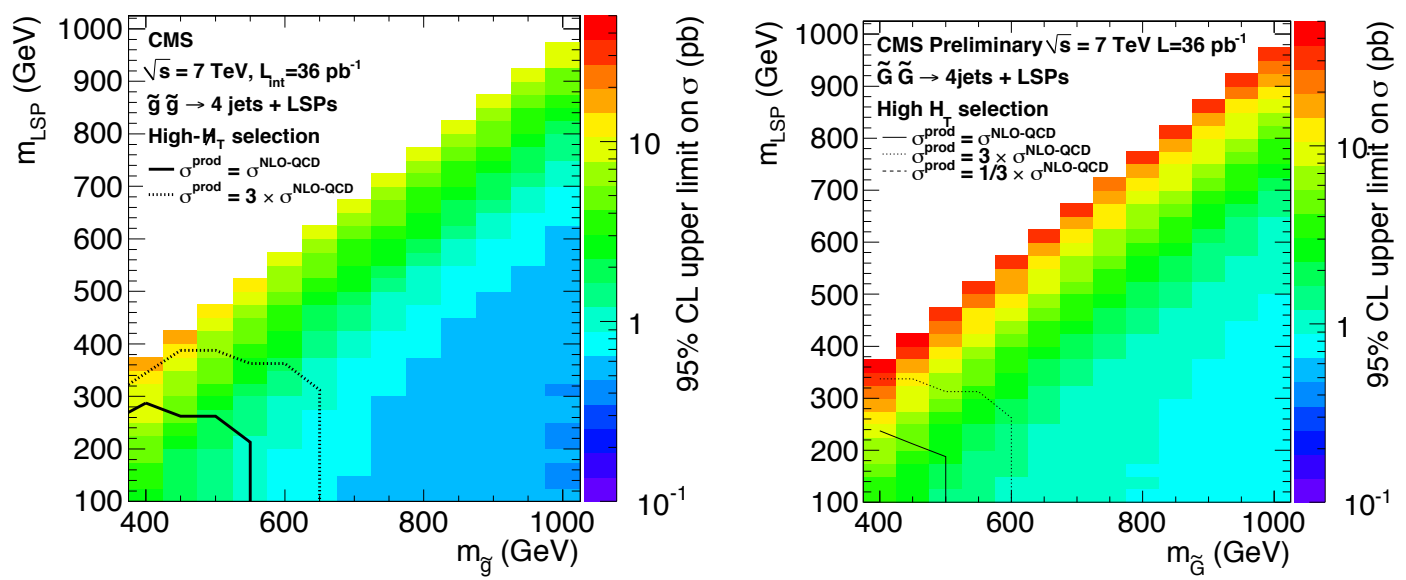

Figure 6.11: The 95\% CL upper limits for gluino pair production cross sections for High- $\mathrm{H}_{\mathrm{T}}$ (left) and High- $\mathrm{H}_{\mathrm{T}}$ (right) selections as a function of the gluino and LSP $\operatorname{mass}[44,70]$.

\subsection{Conclusion}

In this part of the thesis a search for new physics based on multi-jet and large missing transverse momentum is presented. The SM backgrounds are estimated using data-driven technique with minimum reliance on the simulations. Jet resolutions, crucial inputs to the QCD background estimation method, are also measured using data-driven methods from $\gamma+$ jet and dijet events. The main uncertainties on the background estimations comes from the statistical uncertainties of the control samples 

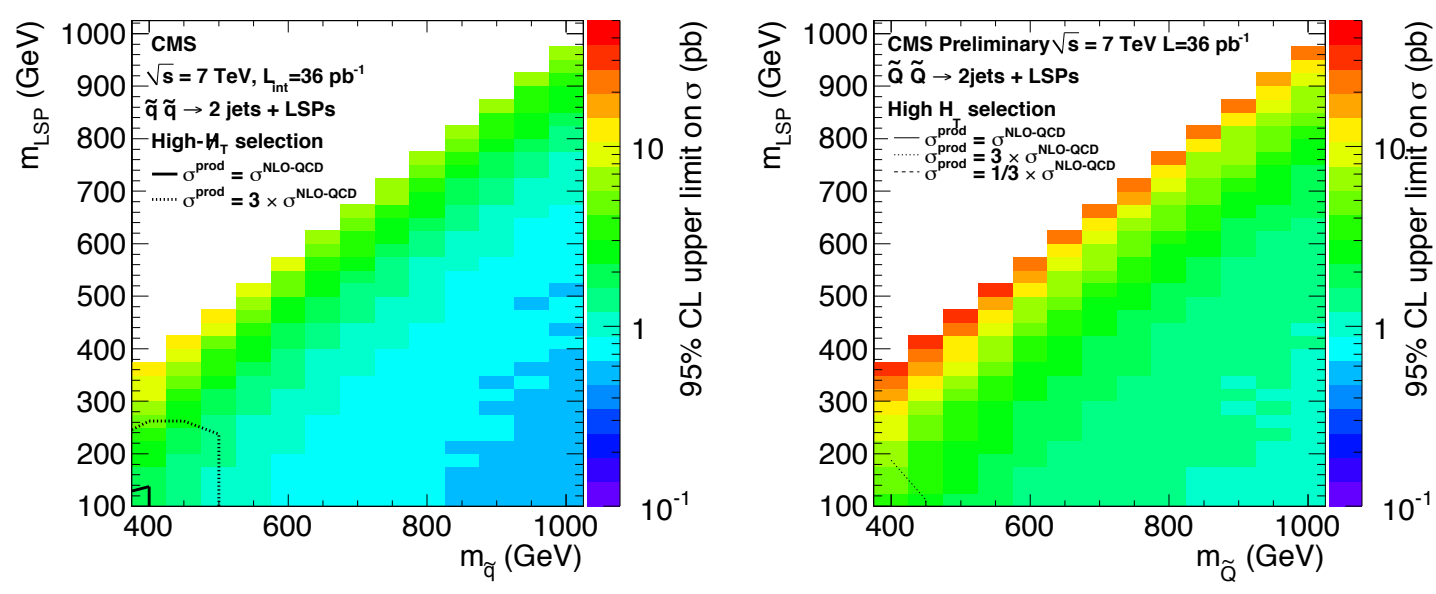

Figure 6.12: The 95\% CL upper limits for squark pair production cross sections for High- $\mathrm{H}_{\mathrm{T}}$ (left) and High- $\mathrm{H}_{\mathrm{T}}$ (right) selections as function of squark and LSP mass $[44,70]$.

used for the background estimation. The event yields observed in data for baseline, High- $\mathrm{H}_{\mathrm{T}}$ and High- $\mathrm{H}_{\mathrm{T}}$ selections are consistent with SM contributions and no excess is observed.

Since no excess observed in data, we derived upper limits in the R-parity CMSSM parameter space with parameters $A_{0}=0, \mu>0$, and $\tan \beta=10$. Depending on the squark and gluino masses, a 95\% CL upper limit on the production cross section of 2-3 pb is obtained [44]. Gluino masses below $500 \mathrm{GeV}$ are excluded with 95\% CL for the squarks with mass below $1 \mathrm{TeV}$. Limits are also set in the context of simplified models and depending on the mass ranges, a 95\% CL upper limit is obtained on the production cross section for the 0.5-30 pb range [44]. 


\section{CHAPTER 7 SUMMARY AND CONCLUSION}

In the first part of thesis, the results of R\&D studies for the CMS Hadronic Endcap Calorimeter are presented. The LHC has two phase upgrade plans for increasing the instantaneous luminosity. The first phase will be completed without any detector upgrade and during the second phase, the expected luminosity will be $\sim 10^{35}$ $\mathrm{cm}^{-2} \mathrm{~s}^{-1}$. The upgraded luminosity conditions will create a high radiation environment and some detector parts, such as the scintillator tiles used in the CMS Hadronic Endcap detectors, will be damaged and loose their efficiencies. As a solution, wavelength shifter (WLS) deposited quartz plates are proposed to replace the current HE scintillators.

After the light efficiency tests performed at the Fermilab and CERN test beam areas, the p-terphenyl (pTp) was determined as the WLS material. $2 \mu \mathrm{m}$ pTp deposited quartz plates increase the light output by at least factor of four compared to plane quartz plates. Both quartz plates and pTp are shown to be radiation hard. The irradiation tests performed on pTp showed that after 40 MRad radiation we still have more than $80 \%$ of the initial light output.

The pTp deposited quartz plates are also tested for their calorimeter capabilities. The test beam results showed that the prototype has a $15 \%$ hadronic energy resolution for a $350 \mathrm{GeV}$ pion beam, and considering the energy leakage from the undersize prototype, this is a promising result. On a larger scale, a pTp deposited quartz plate can be a replacement to the current HE calorimeter, which has $\sim 8 \%$ 
resolution at the $300 \mathrm{GeV}$ pion beam energy.

In the second part of this thesis a search for new physics with multijet and large missing transverse momentum is presented with emphasis on the QCD background estimation methoda based on smearing seed events by measured jet resolutions. After developing the background estimations based on data we chose two search regions based on the observables $H_{\mathrm{T}}$ and $\mathrm{H}_{\mathrm{T}}$ which are central to this inclusive search. Data driven methods are used to measure resolutions and to predict event yields from the SM productions $(\mathrm{QCD}$ multijet, $\mathrm{Z}(\rightarrow \nu \nu)+$ jets, $\mathrm{W}+$ jets and $\mathrm{t} \overline{\mathrm{t}})$. The total number of events we observed in data are 111 for the baseline selection, 14 for the High- $\breve{A}_{\mathrm{T}}$ selection, and 29 for the High- $\mathrm{H}_{\mathrm{T}}$ selection. No excess above the expectations is observed.

Since no excess is observed, we estimated the expected number of signal events by using simulations to derive limits on new physics. The upper limits are obtained in the R-parity conserving CMSSM parameter space. Depending on the gluino and squark masses, a 95\% CL upper limit is obtained on the production cross sections in the 2-3 pb range, and gluino masses below $500 \mathrm{GeV}$ are excluded for squark masses below $1 \mathrm{TeV}$. The results are also interpreted in the context of simplified model spectra where final states are described by pair production of new particles. A 95\% CL upper limit on the production cross section for the $0.5-30 \mathrm{pb}$ range is obtained depending on new particle masses in the decay chains. 


\section{APPENDIX A \\ EFFECT OF LOWER JET $p_{T}$ LIMIT ON RESOLUTION MEASUREMENTS}

Due to limits on the jet energy correction, we have a lower limit on the jet $\mathrm{p}_{\mathrm{T}}$ that can be used in the jet energy resolution measurements. This also determines the $\mathrm{p}_{\mathrm{T}}$ of the particle jet that is used during smearing, since we do not use reconstructed jets below that limit. In this study the lowest jet $\mathrm{p}_{\mathrm{T}} \mathrm{I}$ use is $20 \mathrm{GeV}$. Although the lowest limit is set, there is an uncertainty that changes when we go to this lowest limit. To quantify the contribution to uncertainty due to the jet $\mathrm{p}_{\mathrm{T}}$ limit, I used 20, $42,62,87 \mathrm{GeV}$ requirements on particle jets during the smearing process, and the $\not_{\mathrm{T}}$ distribution is estimated for each case.

The QCD PYTHIA sample is used to measure resolution functions and to

estimate $H_{\mathrm{T}}$ distributions. The resolution functions are measured as $\mathrm{p}_{\mathrm{T}}^{\text {RecJet }} / \mathrm{p}_{\mathrm{T}}^{\text {ParticleJet }}$ for the leading reconstructed jets where reconstructed jets are matched to the particle level jets within a radius of 0.25 in $\eta-\phi$ space $(\Delta R=0.25)$. The resolution functions are measured in different particle jet $\mathrm{p}_{\mathrm{T}}$ and $\eta$ bins.

$H_{\mathrm{T}}$ is calculated using reconstructed jets (nominal $\mathrm{H}_{\mathrm{T}}$ ) and smeared particle jets (estimated $\mathrm{H}_{\mathrm{T}}$ ) with $\mathrm{p}_{\mathrm{T}}>30 \mathrm{GeV}$ and $|\eta|<5$, and compared after the event selections listed in Section 4.4. Particle jets in the QCD samples were smeared by the resolution functions that were measured by the very same QCD sample.

Figure A.1 compares nominal and estimated $H_{\mathrm{T}}$ distributions for a $20 \mathrm{GeV}$ requirement on particle jet $\mathrm{p}_{\mathrm{T}}$. The top plot shows the distributions whereas the 
bottom plot shows their ratio. Except for the $200-300 \mathrm{GeV}$ region (MC weight problem) and the last $\mathrm{p}_{\mathrm{T}}$ bin (not enough number of events) there is a good agreement between the two distributions. Similar distributions for 42,62 , and $87 \mathrm{GeV} \mathrm{p}_{\mathrm{T}}$ requirements are shown in Figures A.2, A.3, and A.4. Increasing the $\mathrm{p}_{\mathrm{T}}$ threshold on smeared particle jets increases the error for estimating the QCD $H_{\mathrm{T}}$ spectrum. If the jet resolutions can be measured for the jets with $\mathrm{p}_{\mathrm{T}}>40 \mathrm{GeV}$ the expected error on estimating the $H_{\mathrm{T}}$ distribution is around $10 \%$ and this error goes up to $20 \%$ if the $\mathrm{p}_{\mathrm{T}}$ threshold for smeared jets is increased to $\sim 80 \mathrm{GeV}$. In data-driven methods, the dijet samples can be used to measure the jet resolution for jets with $\mathrm{p}_{\mathrm{T}} \sim 50 \mathrm{GeV}$, whereas by using the $\gamma+$ jet sample we can measure the resolution for the jets with $\mathrm{p}_{\mathrm{T}} \sim 20 \mathrm{GeV}$. Therefore it is very important to combine dijet and $\gamma+$ jet results to measure resolutions for a wider $\mathrm{p}_{\mathrm{T}}$ spectrum. 


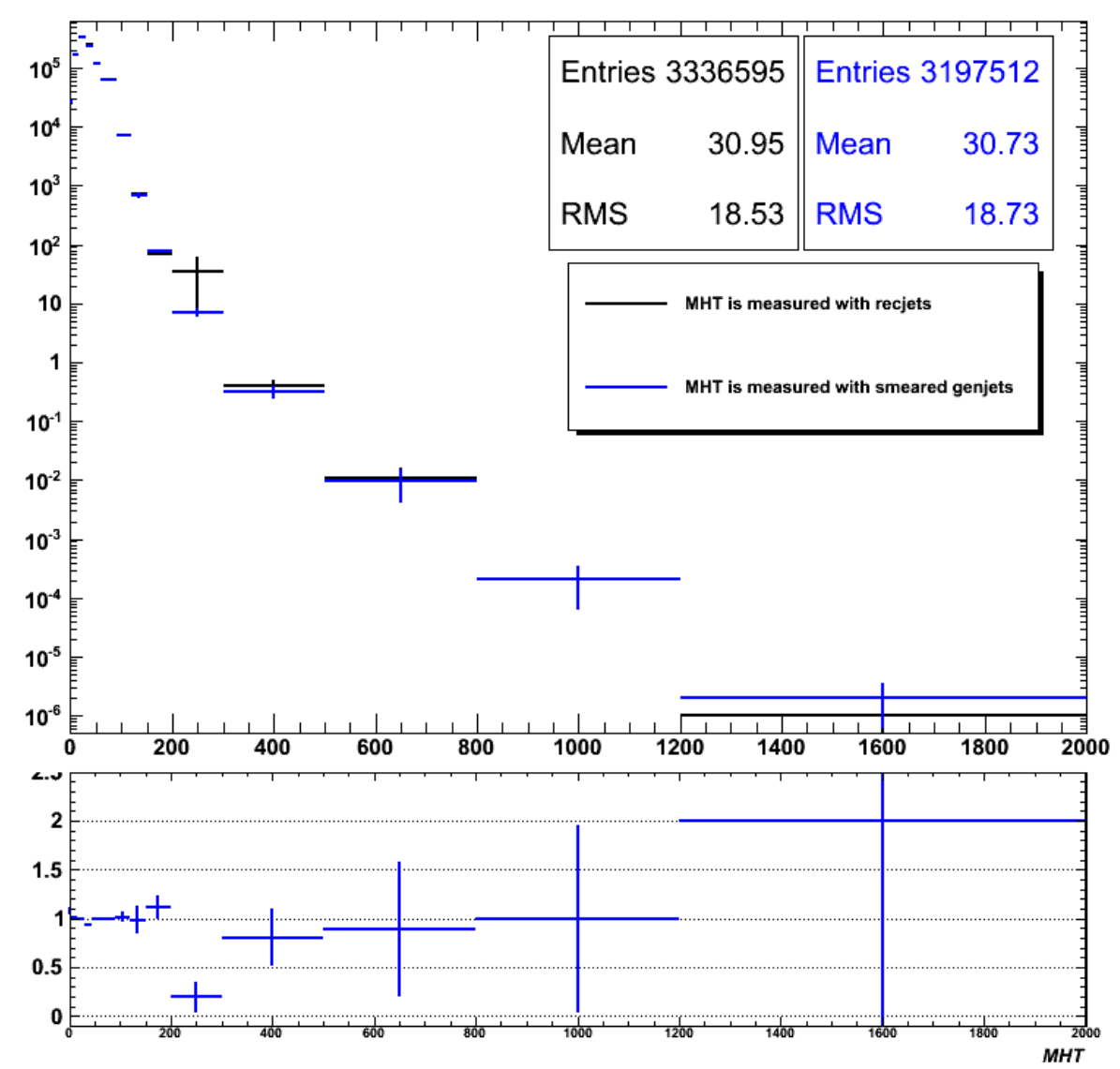

Figure A.1: Comparison of nominal $\bigsqcup_{\mathrm{T}}$ (black) and estimated $\bigsqcup_{\mathrm{T}}$ (blue) distributions. Particle jets with $p_{T}<20 \mathrm{GeV}$ are not smeared. Top plot shows distributions whereas the bottom plot shows their ratio. 

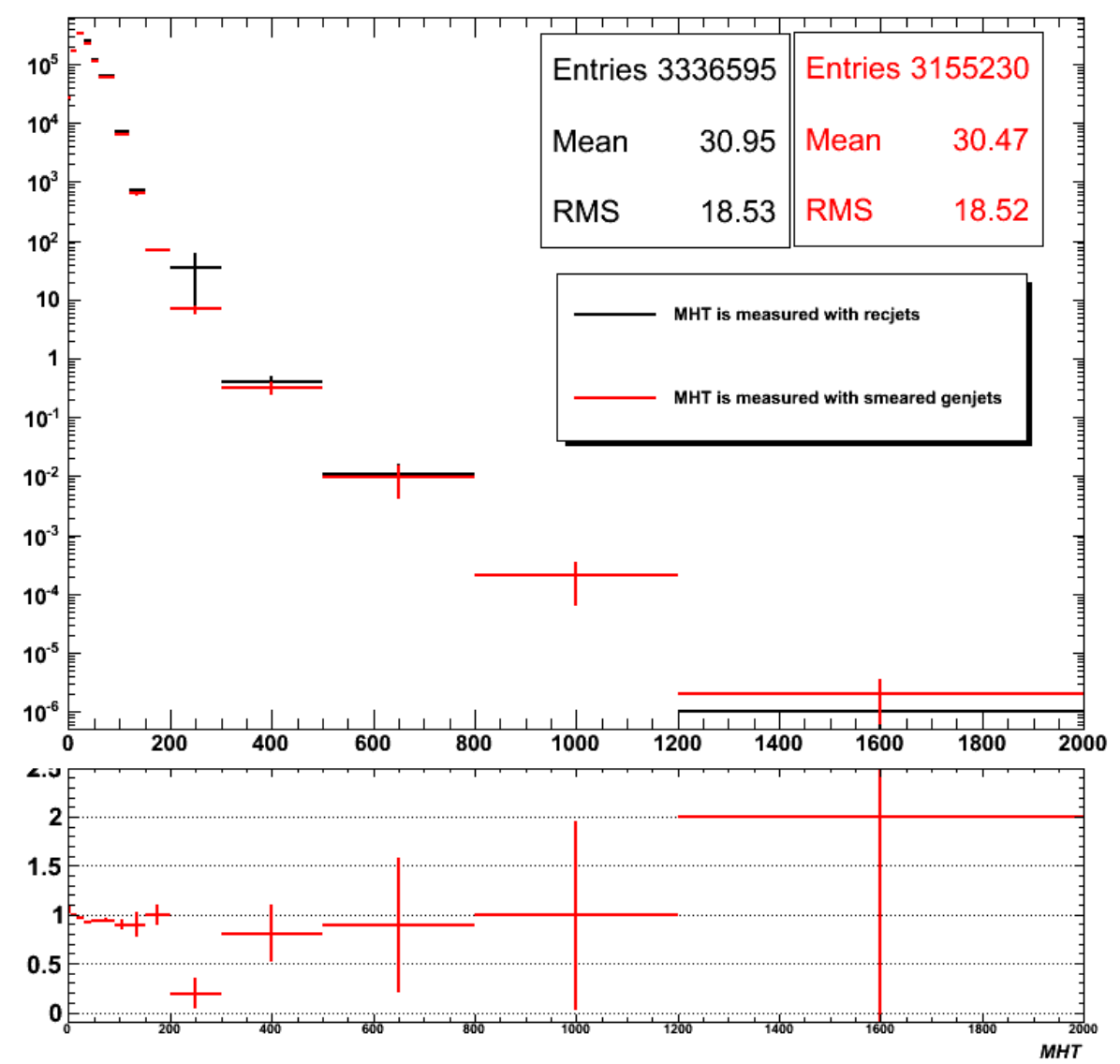

Figure A.2: Comparison of nominal $\not_{\mathrm{T}}$ (black) and estimated $\bigsqcup_{\mathrm{T}}$ (red) distributions. Particle jets with $p_{T}<42 \mathrm{GeV}$ are not smeared. Top plot shows distributions whereas the bottom plot shows their ratio. 

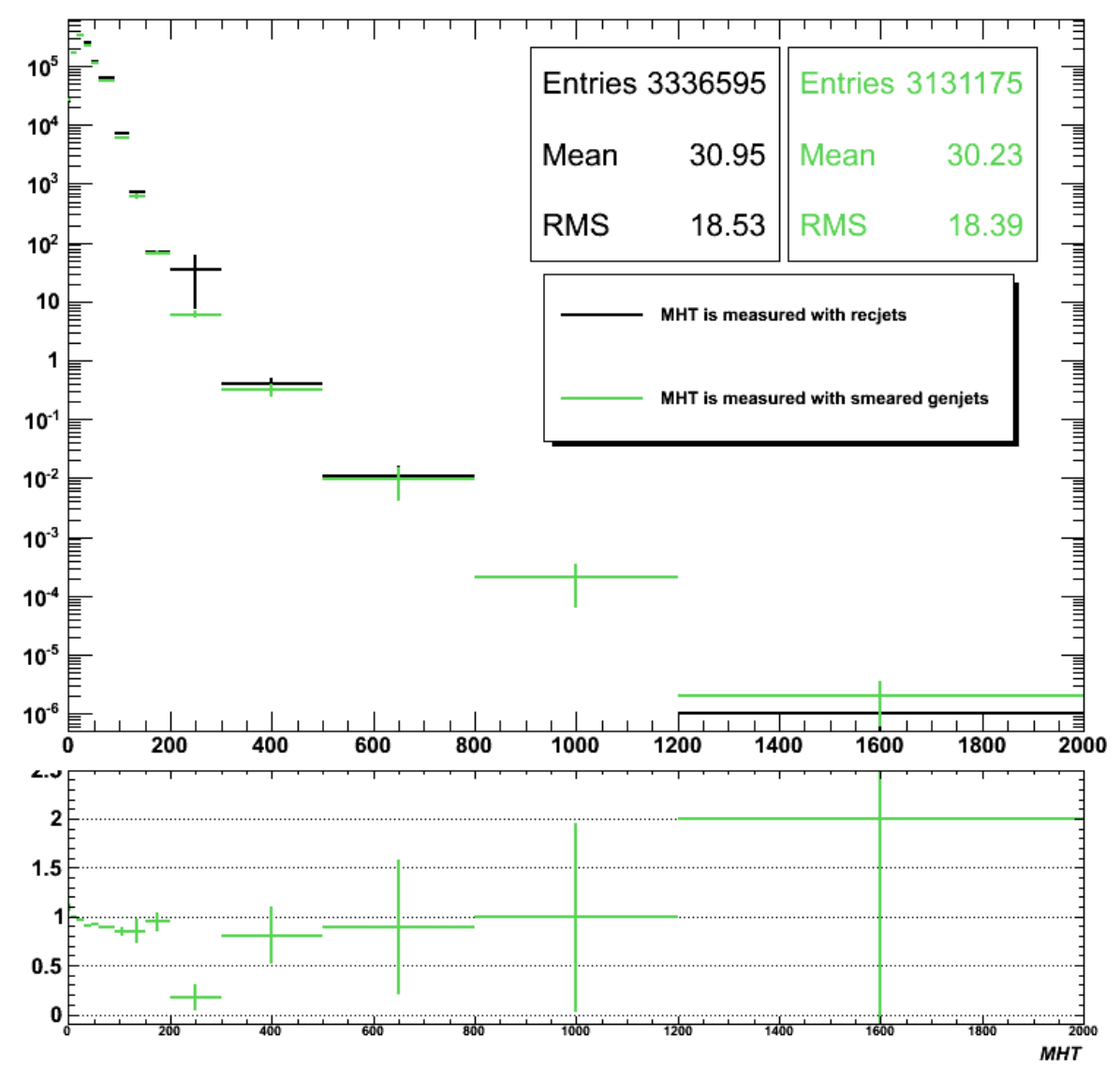

Figure A.3: Comparison of nominal $H_{\mathrm{T}}$ (black) and estimated $H_{\mathrm{T}}$ (green) distributions. Particle jets with $p_{T}<62 \mathrm{GeV}$ are not smeared. Top plot shows distributions whereas the bottom plot shows their ratio. 

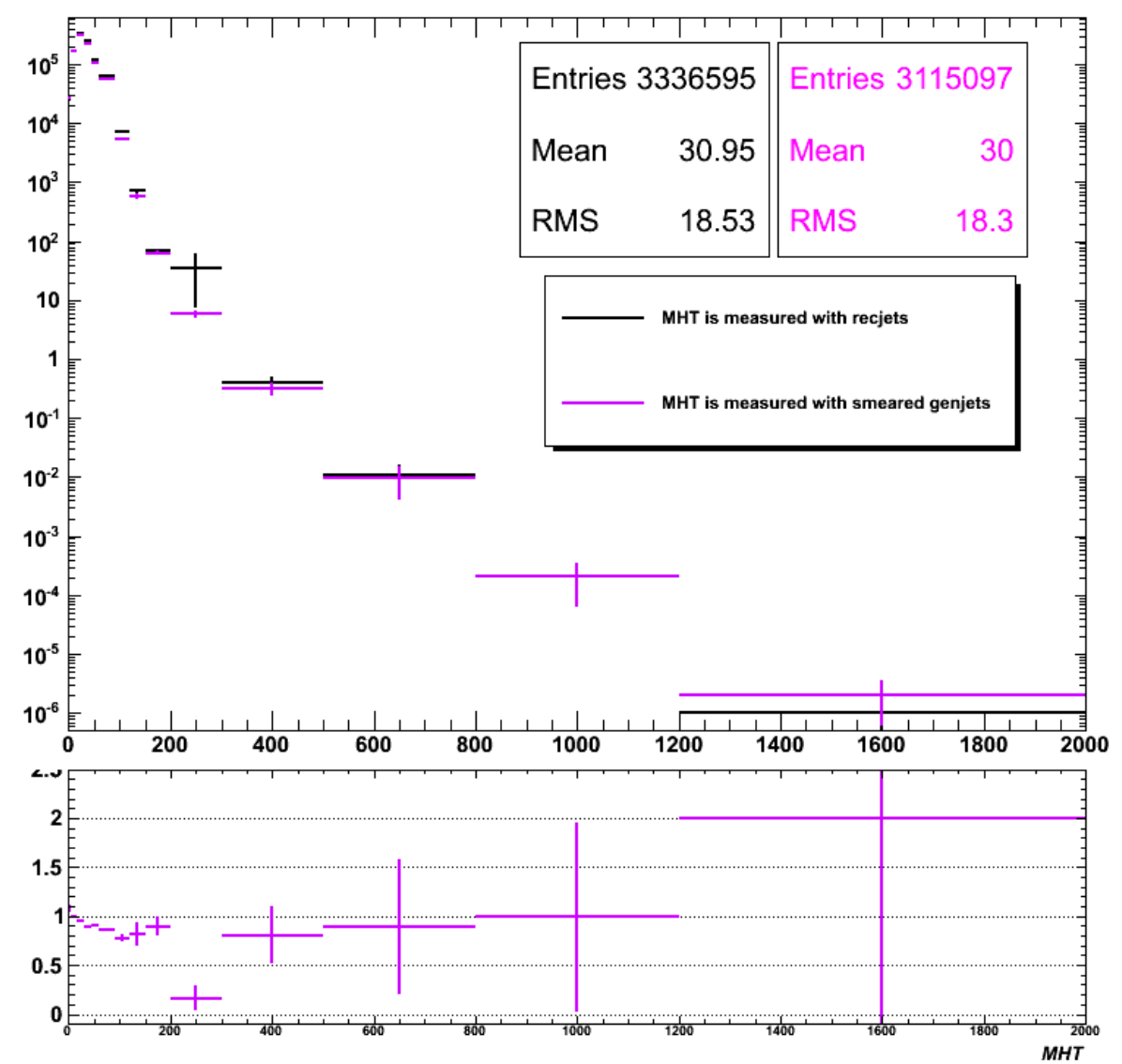

Figure A.4: Comparison of nominal $H_{\mathrm{T}}$ (black) and estimated $H_{\mathrm{T}}$ (violet) distributions. Particle jets with $p_{T}<87 \mathrm{GeV}$ are not smeared. Top plot shows distributions whereas the bottom plot shows their ratio. 


\section{APPENDIX B \\ THE EFFECT OF $\Delta$ R MATCHING ON INTRINSIC RESOLUTION}

The measurement of intrinsic resolution $\left(\mathrm{p}_{\mathrm{T}}^{\mathrm{Jet}} / \mathrm{p}_{\mathrm{T}}^{\text {ParticleJet }}\right)$ is based on matching between reconstructed jets and particle level jets. This matching is done using a $\Delta R$ variable which is defined as $\sqrt{(\Delta \eta)^{2}+(\Delta \phi)^{2}}$. The requirement of $\Delta \mathrm{R}$ can impact the matching between jets and particle-level jets, and therefore can result in a small variation in the intrinsic resolution measurements. This variation is studied using different $\Delta \mathrm{R}$ values $(\Delta \mathrm{R}=0.1,0.15,0.2,0.25$ and 0.30$)$. Figure B.1 shows measured intrinsic resolutions obtained as $\sigma$ of Gaussian fitted region with \pm 2 RMS around the mean of the distributions. There is no significant effect of varying the matching criteria for any $\mathrm{p}_{\mathrm{T}}$ range. The effect of changing the $\Delta \mathrm{R}$ cut is only visible for the lowest $\mathrm{p}_{\mathrm{T}}$ bin $(0-20 \mathrm{GeV})$, and the effect is less than $5 \%$ for all the $\eta$ bins used in this study. 

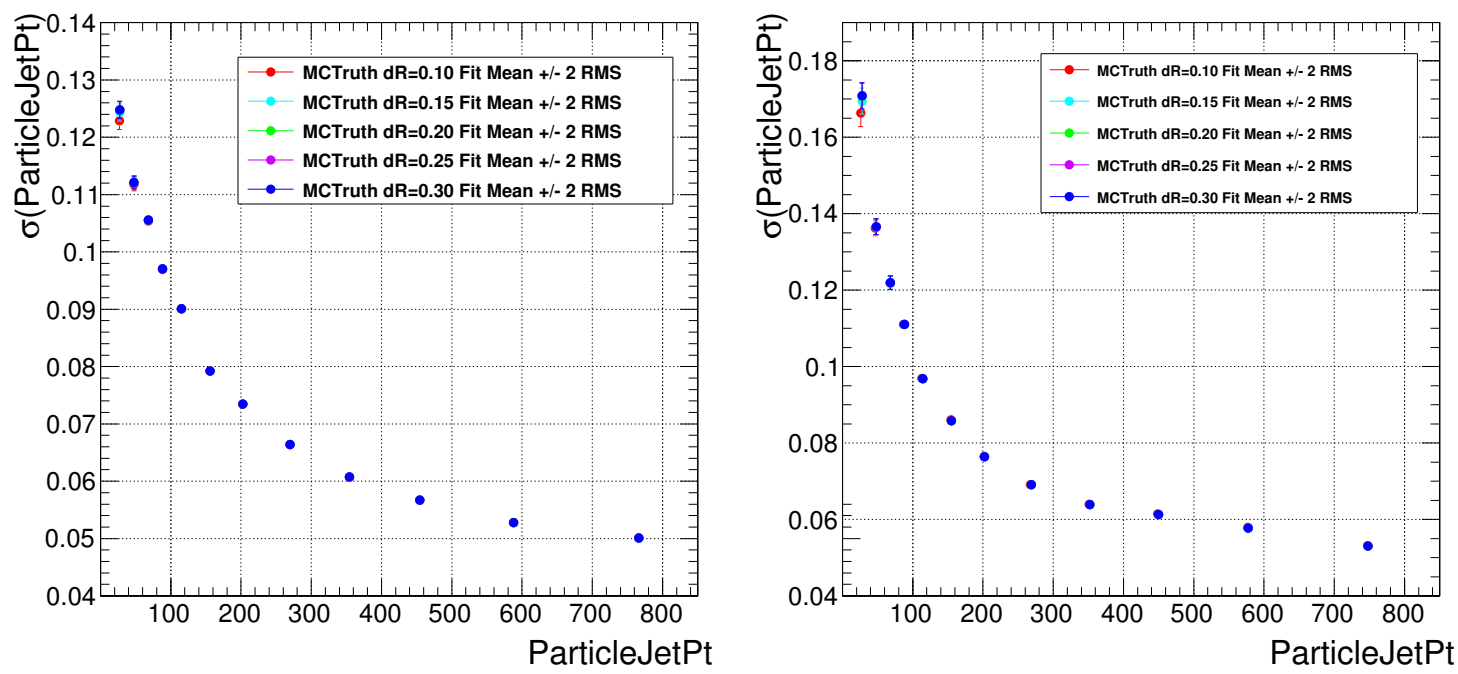

(a) $0.0<\left|\eta^{\text {ParticleJet }}\right|<1.1$

(b) $1.1<\left|\eta^{\text {ParticleJet }}\right|<1.7$
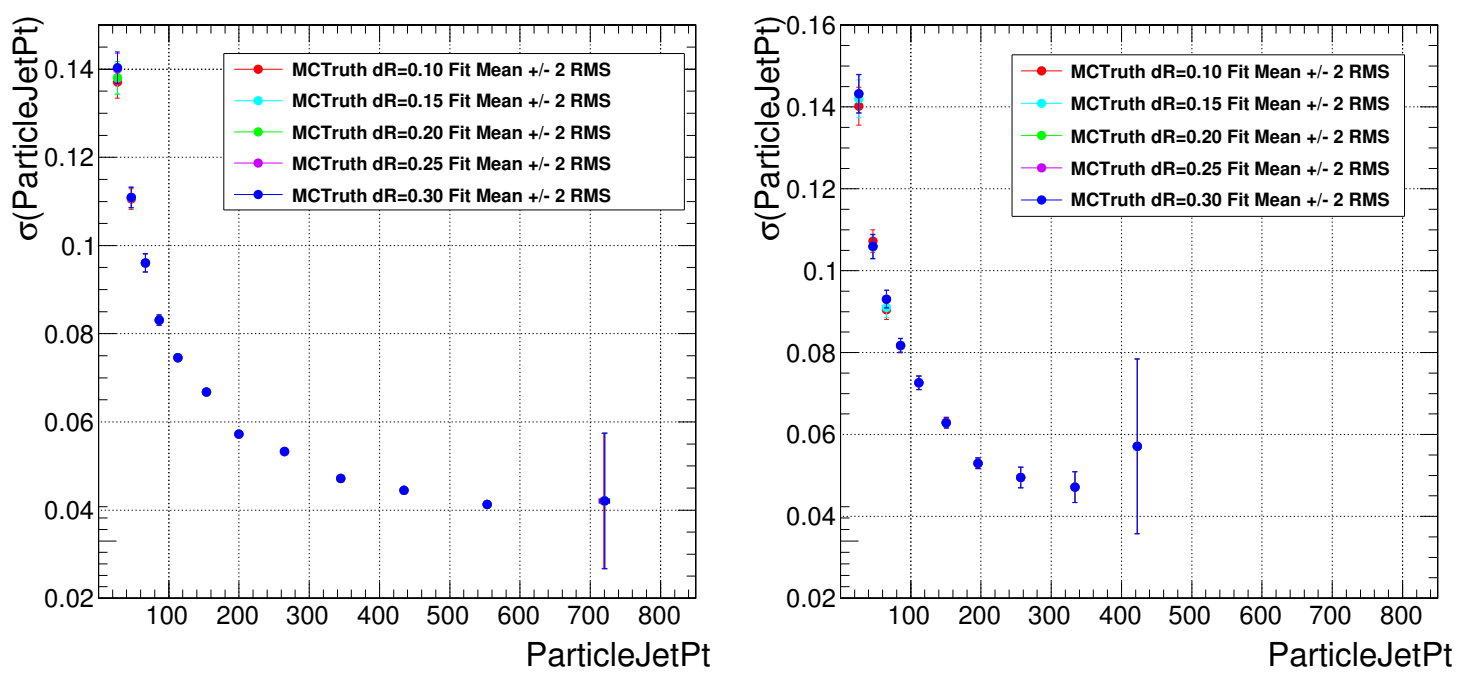

(c) $1.7<\left|\eta^{\text {ParticleJet }}\right|<2.3$

(d) $2.3<\left|\eta^{\text {ParticleJet }}\right|<5.0$

Figure B.1: Effect of varying the $\Delta \mathrm{R}$ matching between leading reconstructed jet and particle jet on intrinsic resolution as a function of particle jet $\mathrm{p}_{\mathrm{T}}$ for the four different eta bins (a) $0.0<|\eta|<1.1$, (b) $1.1<|\eta|<1.7$, (c) $1.7<|\eta|<2.3$, (d) $2.3<|\eta|<5.0$. 


\section{REFERENCES}

[1] The LHC Study Group. Large Hadron Collider. Technical Report 03, 1993.

[2] CMS Colloboration. The CMS Experiment at the CERN LHC. JINST, 0803:S08004, 2008.

[3] See: http://atlas.ch/.

[4] See: http://lhcb.web.cern.ch/lhcb/.

[5] See: http://aliweb.cern.ch/.

[6] See: http://cdsweb.cern.ch/collection/Photos.

[7] CMS Collaboration. CMS technical design report, volume I: Detector Performance \& Software. 2006.

[8] CMS Collaboration. CMS technical design report, volume II: Physics performance. J. Phys., G34:995-1579, 2007.

[9] CMS Collaboration. The Muon Project Technical Design Report. 1997.

[10] CMS Collaboration. The HCAL Technical Design Report. 1997.

[11] See: https://twiki.cern.ch/twiki/bin/view/CMSPublic/LumiPublicResults.

[12] I. Dumanoglu et al. Radiation-hardness Studies of High-OH Content Quartz Fibers Irradiated with $500 \mathrm{MeV}$ Electrons. Nucl. Instrum. Meth., A490:444-455, 2002

[13] K. Cankocak et al. Radiation-hardness Measurements of High-OH Content Fibers Irradiated with $24 \mathrm{GeV}$ Protons up to 1.25 Grad. Nucl. Instrum. Meth., A585:20-27, 2008. 
[14] U. Akgun and Y. Onel. Radiation-Hard Quartz Cerenkov Calorimeters. AIP Conf. Proc., 867:282-289, 2006.

[15] U. Akgun et al. CMS Hadronic EndCap Calorimeter Upgrade Studies for SLHC P-Terphenyl Deposited Quartz Plate Calorimeter Prototype. IEEE Trans. Nucl. Sci., 57:754-759, 2010.

[16] U. Akgun et al. Comparison of PMTs from three different manufacturers for the CMS-HF Forward Calorimeter. IEEE Trans. Nucl. Sci., 51:1909-1915, 2004.

[17] U. Akgun et al. Complete Tests of 2000 Hamamatsu R7525HA Phototubes for the CMS-HF Forward Calorimeter. Nucl. Instrum. Meth., A550:145-156, 2005.

[18] U. Akgun et al. Afterpulse Timing and Rate investigation of Three Different Hamamatsu Photomultiplier Tubes. Journal of Instrumentation, 3:T01001, 2008.

[19] S. Agostinelli et al. GEANT4 - a simulation toolkit. Nucl. Instrum. Meth., A506:250-300, 2003.

[20] J. Allison et al. Geant4 developments and applications. IEEE Trans. Nucl. Sci., 53:270-278, 2006.

[21] R. Wigman. Calorimetry, Energy Measurement in Particle Physics. Oxford University Press, 2000.

[22] K. Nakamura et al. Particle Data Group. J. Phys., G37:075021, 2010 and 2011 partial update for the 2012 edition.

[23] J. Wess and B. Zumino. Supergauge transformations in four dimensions. Nucl. Phys., B70:39, 1974. 
[24] H.-C. Cheng T. Appelquist and B. A. Dobrescu. Bounds on universal extra dimensions. Phys. Rev., D64:035002, 2001.

[25] K. Lane. Two lectures on Technicolor. 2002. [arXiv:0202255v1].

[26] E. A. Paschos C. T. Hill, M. A. Luty. Electroweak symmetry breaking by fourthgeneration condensates and the neutrino spectrum. Phys. Rev., D43:3011, 1991.

[27] S. Ferrara P. Fayet. Phys. Rep, 32:249, 1977.

[28] H. P. Nilles. Phys. Rep, 110:1, 1984.

[29] G. L. Kane He. E. Haber. Phys. Rep, 117:75, 1985.

[30] S. Dawson. SUSY and Such. 1997. [arXiv:hep-ph/9612229v2].

[31] J. Ellis. Supersymmetry for Alp Hikers. 2002. [arXiv:hep-ph/0203114v1].

[32] S. P. Martin. A Supersymmetry Primer. 2011. [arXiv:hep-ph/9709356v6].

[33] G. Ross. Grand Unified Theories. Benjamin/Cummings. 1985.

[34] L. Roszkowski G. L. Kane, C. Kolda and J. D. Wells. Study of constrained minimal supersymmety. Phys. Rev., D58:6173-6210, 1994.

[35] P. Schuster J. Alwall and N. Toro. implified Models for a First Characterization of New Physics at the LHC. Phys. Rev., D79:075020, 2009.

[36] M. Lisanti, J. Alwall, M. P. Le. Searching for gluinos at the Tevatron and beyond. Int. J. Mod. Phys., A23:46374646., 2008.

[37] LHC New Physics Working Group. Simplified Models for LHC New Physics Searches. See: www.lhcnewphysics.org.

[38] CMS Collaboration. Particle Flow Event Reconstruction in CMS and Perfor- 
mance for Jets, Taus and MET. CMS Physics Analysis Summary, PFT-09-001, 2009.

[39] G. P. Salam M. Cacciari and G. Soyez. The anti-kt jet clustering algorithm. JHEP, 0804:063, 2008.

[40] CMS Collaboration. Jet Energy Corrections determination at $7 \mathrm{TeV}$. CMS Physics Analysis Summary, JME-10-010, 2010.

[41] CMS Collaboration. Missing transverse energy performance of the CMS detector. JINST, 6:P09001, 2011.

[42] CMS Collaboration. HCAL performance from first collision data. CMS Detector Performance Summary, DPS-2010-025, 2010.

[43] CMS Collaboration. Electromagnetic calorimeter commissioning and first results with 7 TeVdata. CMS Note, NOTE-2010-012, 2010.

[44] CMS Collaboration. Search for new physics at CMS with jets and missing momentum. JHEP, 1108:155, 2011.

[45] J. Allison et al. Geant4 developments and applications. IEEE 53, 53-1:270278, 2006.

[46] S. Mrenna T. Sjöstrand and P. Z. Skands. PYTHIA 6.4 Physics and Manual. JHEP, 05:026, 2006.

[47] J. Alwall et al. Madgraph/MadEvent v4: The New Web Generation. JHEP, 09:028, 2007.

[48] J. Pumplin et al. New generation of parton distributions with uncertainties from global QCD analysis. JHEP, 07:012, 2002. 
[49] N. Kidonakis. Next-to-next-leading Soft-Gluon Corrections for the Top Quark Cross Section and Transverse Momentum Distribution. Phys. Rev., D82:114030, 2010.

[50] K. Melnikov and F. Petriello. Electroweak Gauge Boson Production at Hadron Colliders through $\mathrm{O}\left(\alpha(s)^{2}\right)$. Phys. Rev., D74:114017, 2006.

[51] R. Hopker W. Beenakker and M. Spira. PROSPINO:A program for PROduction of Supersymmetric Particles In Next-to-leading Order QCD. 2011. [arXiv:1101.1628v2].

[52] CMS Collaboration. Data-Driven Estimation of the Invisible Z Background to the SUSY MET Plus Jets Search. CMS Physics Analysis Summary, SUS-08-002, 2009.

[53] CMS Collaboration. Measurement of the Isolated Prompt Photon Production Cross Section in pp Collisions $\sqrt{s}=7$ TeV. Phys. Rev. Lett., 106:082001, 2010.

[54] CMS Collaboration. Measurement of Inclusive W and Z Cross Sections in pp Collisions at $\sqrt{s}=7 \mathrm{TeV}$. JHEP, 1108:117, 2011.

[55] CMS Collaboration. Observation of $\mathrm{W} \rightarrow \tau \nu$ Production in pp Collisions at $\sqrt{s}=7 \mathrm{TeV}$. CMS Physics Analysis Summary, EWK-11-002, 2011.

[56] UA2 Collaboration. Measurement of Production and Properties of Jets at the CERN anti-p p Collider. Z. Phys., C20:117, 1983.

[57] A. Bhatti et al. Determination of the jet energy scale at the Collider Detector at Fermilab. Nucl. Inst. Meth., A566:375, 2006. 
[58] D0 Collaboration. Determination of the absolute jet energy scale in the D0 calorimeters. Nucl. Inst. Meth., A424:352, 1999.

[59] CMS Collaboration. The Tracker Project Technical Design Report. LHCC 98-6, CMS TDR 5, 1998.

[60] CMS Collaboration. Determination of Jet Energy Calibration and Transverse Momentum Resolution in CMS. submitted to JINST, 2011. [arXiv:1107.4277v1].

[61] G. Marchesini S. Moretti K. Odagiri P. Richardson M. H. Seymour G. Corcella, I. G. Knowles and B. R. Webber. HERWIG 6.5. JHEP, 0101:010, 2001.

[62] S. Kuhlmann H. L. Lai J. F. Owens W. K. Tung J. Huston, E. Kovacs. A Global QCD Study of Direct Photon Production. Phys. Rev., D51:6139-6145, 1995.

[63] CMS Collaboration. Electromagnetic Calorimeter Commissioning and First Results with 7 TeV Data. CMS Physics Analysis Summary, EGM-10-002, 2010.

[64] CMS Collaboration. Absolute Luminosity Normalization. CMS Detector Performance Summary, DP-2011-002, 2011.

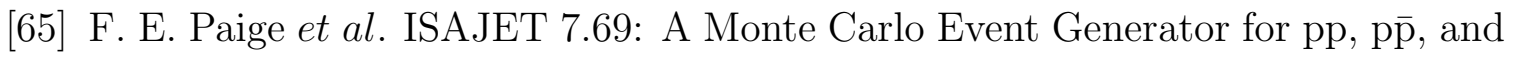
$\mathrm{e}^{+} \mathrm{e}^{-}$Reactions. 2003. [arXiv:hep-ph/0312045v1].

[66] T. Junk. Confidence Level Computation for Combining Searches with Small Statistics. Nucl. Instrum. Meth., A434:435, 1999.

[67] A. L. Read. Presentation of Search Results: The CL(s) Technique. J. Phys., G28:2963, 2002 .

[68] CMS Collaboration. Search for Supersymmetry in pp Collisions at $7 \mathrm{TeV}$ in Events with Jets and Missing Transverse Energy. Phys. Lett., B698:196, 2011. 
[69] L. Randall and D. Tucker-Smith. Dijet Searches for Supersymmetry at the LHC. Phys. Rev. Lett., 101:221803, 2008.

[70] https://twiki.cern.ch/twiki/bin/view/CMSPublic/PhysicsResultsSUS10005. 